\title{
A FAR ULTRAVIOLET IMAGER FOR THE INTERNATIONAL SOLAR-TERRESTRIAL PHYSICS MISSION
}

\author{
M. R. TORR \\ Payload Projects Office, Marshall Space Flight Center, Huntsville, Al 35812, US.A. \\ D. G. TORR and M. ZUKIC \\ Optical Aeronomy Laboratory, University of Alabama in Huntsville, Huntsville, AL 35899, US. A. \\ R. B. JOHNSON \\ Optical E.T.C., Inc., Huntsville, AL 35801, US. A. \\ J. AJELLO \\ JPL, Pasadena, CA, US.A. \\ P. BANKS \\ University of Michigan, Ann Arbor, MI, US.A. \\ K. CLARK \\ University of Washington, Seattle, WA US.A. \\ K. COLE \\ LaTrobe University, La Trobe, Australia \\ C. KEFFER \\ Optical Aeronomy Laboratory, University of Alabama in Huntsville, AL 35899, US.A. \\ G. PARKS \\ University of Washington, Seattle, WA US.A. \\ B. TSURUTANI \\ JPL, Pasadena, CA, US.A. \\ and \\ J. SPANN \\ Space Science Laboratory, Marshall Space Flight Center, Huntsville, AL 35812, US. A.
}

(Received 2 November, 1994)

\begin{abstract}
The aurorae are the result of collisions with the atmosphere of energetic particles that have their origin in the solar wind, and reach the atmosphere after having undergone varying degrees of acceleration and redistribution within the Earth's magnetosphere. The global scale phenomenon represented by the aurorae therefore contains considerable information concerning the solar-terrestrial connection. For example, by correctly measuring specific auroral emissions, and with the aid of comprehensive models of the region, we can infer the total energy flux entering the atmosphere and the average energy of the particles causing these emissions. Furthermore, from these auroral emissions we can determine the ionospheric conductances that are part of the closing of the magnetospheric currents through the ionosphere, and from these we can in turn obtain the electric potentials and convective patterns that are an essential element to our understanding of the global magnetosphereionosphere-thermosphere-mesosphere. Simultaneously acquired images of the auroral oval and polar cap not only yield the temporal and spatial morphology from which we can infer activity indices, but in conjunction with simultaneous measurements made on spacecraft at other locations within the magnetosphere, allow us to map the various parts of the oval back to their source regions in the magnetosphere. This paper describes the Ultraviolet Imager for the Global Geospace Sciences portion of the International Solar-Terrestrial Physics program. The instrument operates in the far ultraviolet (FUV) and is capable of imaging the auroral oval regardless of whether it is sunlit or in darkness. The instrument has an $8^{\circ}$ circular field of view and is located on a despun platform which
\end{abstract}


permits simultaneous imaging of the entire oval for at least 9 hours of every 18 hour orbit. The three mirror, unobscured aperture, optical system $(f / 2.9)$ provides excellent imaging over this full field of view, yielding a per pixel angular resolution of 0.6 milliradians. Its FUV filters have been designed to allow accurate spectral separation of the features of interest, thus allowing quantitative interpretation of the images to provide the parameters mentioned above. The system has been designed to provide ten orders of magnitude blocking against longer wavelength (primarily visible) scattered sunlight, thus allowing the first imaging of key, spectrally resolved, FUV diagnostic features in the fully sunlit midday aurorae. The intensified-CCD detector has a nominal frame rate of $37 \mathrm{~s}$, and the fast optical system has a noise equivalent signal within one frame of $\sim 10 R$. The instantaneous dynamic range is $>1000$ and can be positioned within an overall gain range of $10^{4}$, allowing measurement of both the very weak polar cap emissions and the very bright aurora. The optical surfaces have been designed to be sufficiently smooth to permit this dynamic range to be utilized without the scattering of light from bright features into the weaker features. Finally, the data product can only be as good as the degree to which the instrument performance is characterized and calibrated. In the VUV, calibration of an an imager intended for quantitative studies is a task requiring some pioneering methods, but it is now possible to calibrate such an instrument over its focal plane to an accuracy of $\pm 10 \%$. In summary, very recent advances in optical, filter and detector technology have been exploited to produce an auroral imager to meet the ISTP objectives.

\section{Introduction and Background}

Energetic charged particles from the solar wind that are swept up by the Earth's magnetic field are accelerated by the various electromagnetic processes operative within the magnetosphere, and large fluxes of these particles (equivalent to 100 million kilowatts during the almost daily events known as magnetic substorms) are channeled down into the atmosphere at high latitudes. The resulting collisions between the energetic particles and the atmospheric gases produce the aurorae. Typically, the aurorae occur around a magnetic field line footprint that is roughly centered on a geomagnetic latitude of $70^{\circ}$, but displaced to lower latitudes at local magnetic midnight, and to higher than the average latitude in the noon sector. The effect is that of a band of emissions that to first order is circular in magnetic co-ordinates, but with these displacements is known as the auroral oval. Imaging of this oval from space provides spatial/temporal information that maps back to different regions of the magnetosphere, provides information on the total energy influx, the acceleration processes, and (with careful selection and interpretation of emission features) the characteristic energy of the incoming particles (Germany et al., 1990, 1994a, b). The solar wind particles flowing within the magnetosphere form giant current systems that close by flowing through the ionosphere. For example, one of these systems, equivalent to approximately 2 million amperes, flows from the magnetosphere, down the magnetic field lines until it finds the high conductance path provided by the ionosphere, flows through the ionosphere along the low latitude boundary of the auroral oval, and then back up the field lines into the magnetosphere. Other current systems follow other paths. The ionosphere is therefore an essential element in the closing of these massive current loops. The potential fields and convective patterns and associated currents are among the last 
Day $356 \quad A p=20 \quad F 10.7=110$

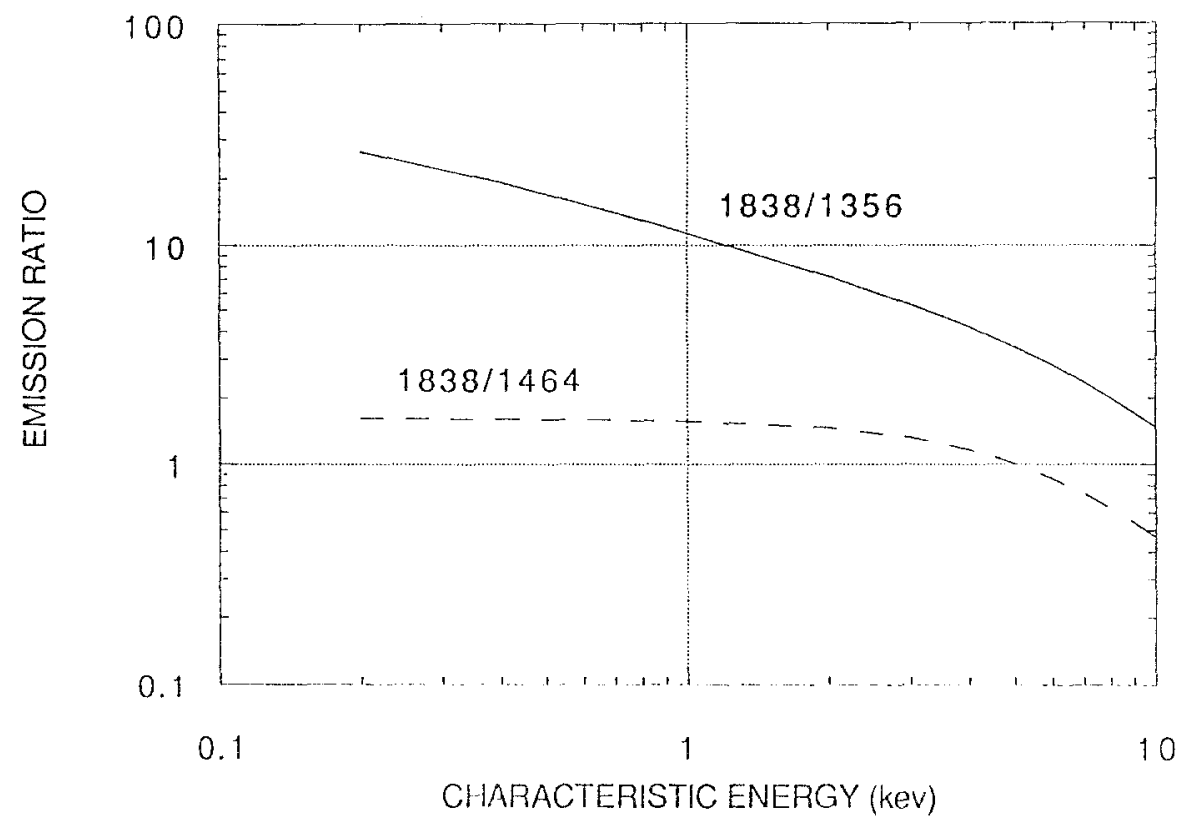

Fig. 1.1. Intensity ratio of two pairs of short/long wavelength features (OI 1356/LBH 1838 and LBH 1464/LBH 1838) as a function of characteristic energy of the precipitating particles.

major missing elements in our ability to model the moment to moment development of the global ionosphere and thermosphere at the present time. By careful interpretation, properly acquired auroral images can be converted into images of ionospheric conductance (Germany et al., 1994b). Auroral images therefore represent a means of tying together the way in which the solar influences are transferred to the magnetosphere via the ionosphere, and provide the information necessary to advance modeling of the ionosphere/thermosphere/mesosphere to its next logical step.

Appropriate modeling tools combined with the new technical capabilities will allow us to make a fundamental step forward in our ability to transform the auroral images into images of primary parameters that describe the precipitating particles (e.g. flux and characteristic energy). Once the relevant spectral features can be isolated from other emissions, these emissions can be used for quantitative science. The concept is as follows: By selecting an emission that is produced in collisions of energetic particles with the atmosphere without subsequent absorption of the photons or quenching of the excited state, use can be made of the fact that the intensity is proportional to the total energy influx. A spatial image of this emission is a spatial image of the total energetic particle influx. Such images will provide the temporal and spatial behavior of the aurora, can be used to relate regions of the aurora to regions in the magnetosphere, and can be used to identify periods of 


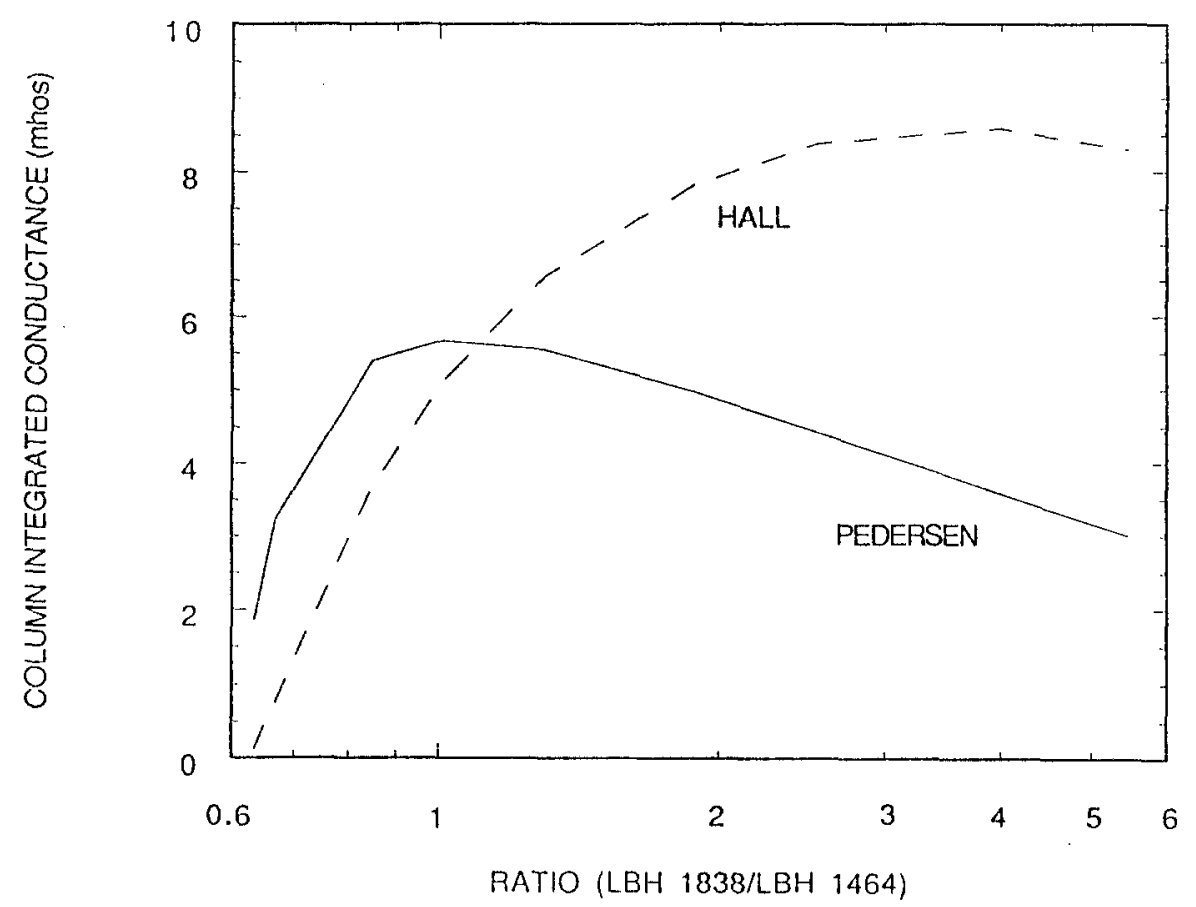

Fig. 1.2. Column-integrated Hall and Pedersen conductances as a function of the emission ration of auroral $\mathrm{LBH}$ at $1838 \mathrm{~A}$ and $1464 \mathrm{~A}$.

interest for overall study by larger groups. Further, by selecting emissions that have very different behavior as a function of altitude, we can estimate the characteristic energy of the precipitating particles. For example, the Lyman-Birge-Hopfield system of bands results from electron impact excitation of $\mathrm{N}_{2}$ :

$$
\begin{aligned}
& \mathrm{N}_{2}\left(X^{1} \Sigma_{g}^{+}\right)+e^{*} \rightarrow \mathrm{N}_{2}\left(a^{1} \Pi_{g}\right)+e, \\
& \mathrm{~N}_{2}\left(a^{1} \Pi_{g}\right) \rightarrow \mathrm{N}_{2}\left(X^{1} \Sigma_{g}^{+}\right)+h \nu_{\mathrm{LBH}} .
\end{aligned}
$$

This system is strongly absorbed by $\mathrm{O}_{2}$ near $1400 \mathrm{~A}$ and the absorption is negligible near 1700A. The ratio of bands near 1400 to bands near $1700 \mathrm{~A}$ is therefore a valuable diagnostic. For very soft particles, which do not penetrate very deep into the atmosphere, the ratio can be normalized, say, to one. Then for very energetic particles which do penetrate deep into the atmosphere, where the $\mathrm{O}_{2}$ absorption at the shorter wavelengths is significant, the longer wavelength emission will be brighter and the ratio will be much less than unity. This is illustrated in Figure 1.1 which shows how the ratio of a long and short wavelength LBH band varies with the characteristic energy of the precipitating particle. Figure 1.2 shows how the column-integrated Hall and Petersen conductances vary as a function of the LBH long to short wavelength intensity ratio. The longer wavelength, nonabsorbed LBH emission serves as the total energy indicator discussed above. Other 


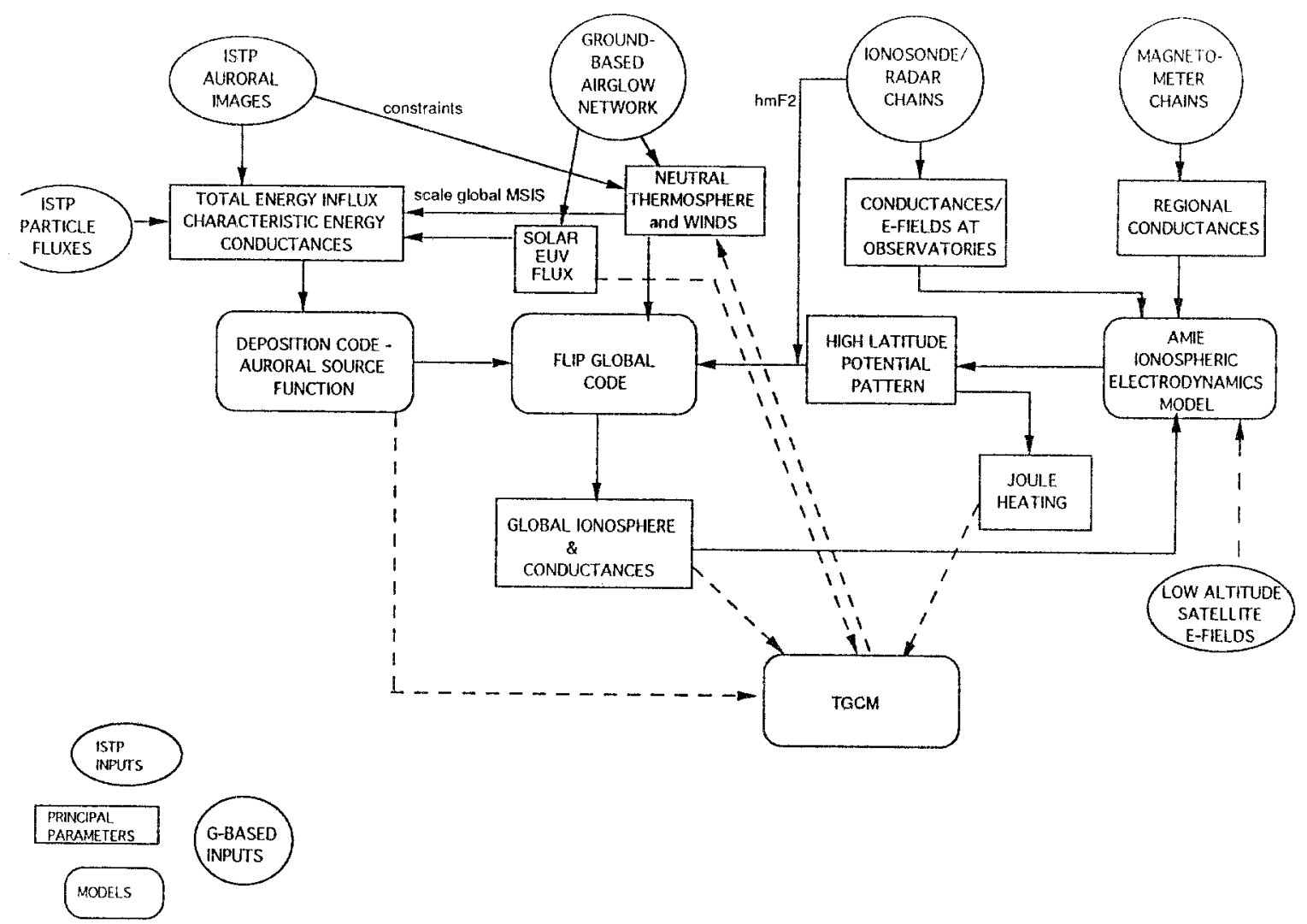

Fig. 1.3. Illustration of the modeling/measurement plan for advancing the understanding of the global ionosphere and electrodynamics.

emissions are measured to provide the constraints on the atmospheric model used to establish the parametric fits. Of course, the success of the data interpretation lies in the degree to which one can globally model the auroral emissions and to the extent to which one can successfully remove the underlying and often comparable airglow. These tasks require sophisticated auroral/airglow tools.

In general, FUV images, such as those discussed here, can be used to infer the properties and origins of processes taking place within the magnetosphere, and can be used to greatly improve our ability to model the global ionosphere and thermosphere. Let us consider an example in the latter category: As was mentioned above, the solar wind - magnetosphere dynamo produces large scale currents in the magnetosphere that ultimately close by flowing through the ionosphere. The high latitude potentials that result cause the ionospheric ions to be convected in response to the magnetospheric processes. The precipitation of charged particle energy and convective electric fields force both the ionosphere and neutral atmosphere (thermosphere and upper mesosphere), resulting in winds, strong heating, and 
composition changes. To date the particle and convective fields have represented major missing elements of our modeling ability and have, by necessity, been assumed or checked only by point to point in situ measurements from orbit or limited field of view imagers. With databases such as that promised by ISTP, we will have the potential to generate the particle and Joule heating as well as the high latitude convection patterns of the ionosphere on a global scale for the first time.

How this might be done is illustrated in Figure 1.3. Starting at the top left, the Far Ultraviolet Imager will provide global images (in which all pixels are exposed simultaneously) of the sunlit and dark auroras. With the appropriate knowledge of the thermosphere, these can be transformed into images of energy influx and characteristic energy of the precipitating particles, and estimates of integrated ionospheric conductance maps. At the lower altitudes of the POLAR spacecraft (i.e., below the acceleration regions), it will be possible to verify these parameter images against the direct particle measurements made at the spacecraft. With this information inferred from the images and an auroral energy deposition code, estimates of the ionospheric source function (ionization rate) can be computed. This information can then be used by a global ionospheric code to compute the global electron concentrations. Having the electron concentrations, we can compute the conductance, a fundamentally important parameter, because this tells us how the magnetospheric currents close through the ionosphere, and allows mapping of ionospheric electric fields to the magnetosphere.

However, the modeling necessary to reach this point is dependent on models of the neutral atmosphere, the neutral winds, convection electric fields and the solar extreme ultraviolet flux; all of which are parameters that will not be measured by ISTP and some of which will not be measured at the same time as the POLAR measurements are made. We can obtain confirmation on the first of these, the concentrations of the major neutral species, at single point locations by making coordinated airglow measurements from the ground. These values can be used to scale the global MSIS model atmosphere. Similarly, from measurements of the peak height of the $F_{2}$ layer $\left(h_{m} F_{2}\right)$ from ground-based ionosonde stations, we can extract the meridional component of the neutral winds (Richards, 1991). As has been mentioned above, actual measurements of the solar EUV flux are most important. At the present, there is no plan to provide these during the POLAR operating period, so that ground based determinations (Fennelly et al., 1994) and models (Richards et al., 1994) will have to be used.

A statistical model is used to obtain the high latitude convection electric fields. Once we have the ionospheric conductances derived from the images, these conductance images can be used as input to an ionospheric electrodynamics model, such as the AMIE (Kamide and Richmond, 1982), to compute the high latitude potential pattern. This is now fed back into the ionospheric code, which computes the ionospheric convection and electron densities, as an additional constraint on the conductance calculations. Ultimately, this may be compared with drift meter data from low altitude satellites. 
TABLE 1.1

Summary of various auroral imagers

\begin{tabular}{|c|c|c|c|c|c|c|}
\hline Imager & $\begin{array}{l}\text { Angular } \\
\text { resolution } \\
\text { deg }\end{array}$ & $\begin{array}{l}\text { Spacecraft } \\
\text { altitude }\end{array}$ & $\begin{array}{l}\text { Wavelength } \\
\AA\end{array}$ & $\begin{array}{l}\text { Image } \\
\text { frame } \\
\text { rate }\end{array}$ & $\begin{array}{l}\text { Noise } \\
\text { equiv. } \\
\text { signal }^{\text {a }}\end{array}$ & Reference \\
\hline UVI/ISTP & 0.03 & $1-8 R_{\mathrm{E}}$ & $\begin{array}{l}4 \text { filters } \\
1300 \text { to } 1900 \\
\text { and one solar } \\
\text { spectral filter } \\
\sim 2000 \AA\end{array}$ & $37 \mathrm{~s}$ & $10 R$ & this paper \\
\hline ISIS-2 & 0.4 & $1400 \mathrm{~km}$ & $3914 / 5577$ & $100 \mathrm{~min}$ & $300 R$ & Anger et al. (1973) \\
\hline DMSP & 0.25 & $850 \mathrm{~km}$ & $4000-11300$ & $100 \mathrm{~min}$ & & Rogers et al. (1974) \\
\hline $\begin{array}{l}\text { KYOKKO } \\
\text { (EXOS-A) }\end{array}$ & 0.3 & $650-4000 \mathrm{~km}$ & $1200-1400$ & $128 \mathrm{~s}$ & & $\begin{array}{l}\text { Kaneda et al. (1977), } \\
\text { Hirao and Itoh (1978) }\end{array}$ \\
\hline $\mathrm{DE}$ & 0.29 & $1-4 R_{\mathrm{E}}$ & $\begin{array}{l}\text { several filters: } \\
1216 \text { to } 6300\end{array}$ & $12 \mathrm{~min}$ & $1 \mathrm{KR}$ & Frank et al. (1981) \\
\hline HILAT & 1.7 & $830 \mathrm{~km}$ & $\begin{array}{l}\text { scanning } \\
\text { spectrometer: } \\
1100 \text { to } 1900\end{array}$ & $100 \mathrm{~min}$ & $25 R-60 R$ & $\begin{array}{l}\text { Meng and Huffman } \\
\text { (1984) }\end{array}$ \\
\hline Viking & 0.08 & $15000 \mathrm{~km}$ & 2 filters: & $20 \mathrm{~s}$ & & Anger et al. (1987) \\
\hline EXOS-D & 0.1 & $300-10000 \mathrm{~km}$ & $\begin{array}{l}2 \text { channels: } \\
1150-1600 ; \\
5577\end{array}$ & $8-16 s$ & $\begin{array}{l}300 R @ 1216, \\
50 R @ 5577\end{array}$ & Oguti et al. (1990) \\
\hline FREJA & 0.08 & $600-1700 \mathrm{~km}$ & $\begin{array}{l}2 \text { filters: } \\
\text { 1340-1800 }\end{array}$ & $6 s$ & $700 R$ & $\begin{array}{l}\text { Murphree } \\
\text { (private comm.) } \\
1993\end{array}$ \\
\hline
\end{tabular}

a Threshold at which the signal is equal to the instrument noise in an integration period.

Going further, once we have the high latitude potential pattern, we are able to compute the Joule heating of the ionosphere, which is comparable in magnitude to the heating caused directly by the precipitating energetic particles. The Joule heating, together with the auroral source function, the global ionosphere and conductances, when fed into a thermospheric global circulation model allows us to model the winds and composition changes in the neutral atmosphere that result from the magnetospheric-solar wind processes.

In summary, for the first time, we will have the potential to model the moment to moment evolution of the global ionosphere/thermosphere in response to magnetospheric forcing. However, what has been briefly outlined above represents a major and ambitious cooperative effort that will require considerable co-ordination to bring about. While pieces of this overall picture have been worked on by different groups, it has not been possible previously to link together all the inputs and tools. 
The energetic particle collisions produce emissions throughout the spectrum from the X-ray regime to the infrared. However, the field of quantitative auroral imaging is still relatively new, despite rapid advances in qualitative interpretation of imaging data over the past two decades. To date much of the work has been in the visible, and almost all restricted to nightside or twilight auroras because of the severe limitations imposed by the bright scattered sunlight on the dayside of the Earth. Ultraviolet images have been made but have been limited to conditions of large solar zenith angles or to relatively intense or unresolved spectral features on the dayside. Even at FUV wavelengths, the problem of scattered long wavelength sunlight is a formidable one. The state-of-the-art of filtering in the FUV has been rather crude until the development of this instrument. The use of the previously available broadband filters in earlier instruments rendered the quality of the images taken by those instruments unsuitable for quantitative interpretation. Furthermore, results that have appeared in the published literature to date are from instruments that have flown on missions that have not had a despun platform capability. Instead of being able to acquire coherent 2-D images as will be possible from ISTP, the earlier images have been built up through various combinations of spacecraft spin, orbital motion, and mirror scanning. In most cases this meant that the dwell time per spatial element was rather short, with correspondingly high thresholds of detection. A summary of previous orbiting auroral instruments is given in Table 1.1. A despun platform and 2-D focal plane detector array permits staring at a fixed location with the result that integration periods can be optimized, allowing images to be simultaneously acquired. A carefully designed instrument, operating in the far ultraviolet and fully utilizing advanced technology, can provide information on both the sunlit and dark auroras, vastly increasing the scope and value of the data for the global studies now needed. This paper describes the Far Ultraviolet Imager (UVI) for the POLAR spacecraft of the Global Geospace Science (GGS) portion of the International Solar-Terrestrial Physics (ISTP) program. The UVI is designed to address these global issues.

\section{Instrument Design}

\subsection{REQUIREMENTS}

Before describing the instrument, we will briefly set out our goals for the capability required of an imager suitable for the ISTP objectives.

The UV Imager is required to achieve simultaneous two-dimensional imaging of the auroral oval. To do this, the imager will be mounted on the single axis despun platform of the POLAR spacecraft. The despun platform is provided as a spacecraft system and can be maintained in a nadir pointing mode, or stepped in $1^{\circ}$ increments to a selected offset angle.

To provide data from which the required auroral parameters can be inferred, the imager must be capable of resolving and measuring several specific emission 
features in the far ultraviolet (FUV), and measuring these features under both sunlit and nightside conditions, which means measuring these features in the presence of the sunlit Earth and lower atmosphere, and airglow. These requirements, in turn, demand excellent stray light rejection, excellent out-of-band light rejection, and relatively narrowband FUV interference filters. In addition to the above, the instrument must be capable of measuring very bright signals $(>100 \mathrm{kR})$ in the auroral oval and very weak signals $(<20 \mathrm{R})$ in the polar cap. On many occasions it will be necessary to measure weak regions simultaneously with bright regions in the same field of view. This requirement further drives the requirement for unusually low scattered light properties in the instrument and also means that the instrument must be able to operate over a large dynamic range in signal intensities. An instantaneous dynamic range of 1000 together with the capability to move this window over the $10^{4}$ overall dynamic range is required.

The time scales of variations occurring within the aurora range from milliseconds to hours. The frame rate of an orbiting imager is primarily dependent on two factors; the datarate available for telemetering the images to the ground, and the number of counts generated by the brightness of the aurora. In the case of the spacecraft used here and with the emphasis on coherent global imaging, the telemetry rate is the principal factor. In view of this, the instrument has been designed to address time scales of the order of $30 \mathrm{~s}$ or longer.

The instrument must operate from a spacecraft in a highly eccentric orbit $(\sim$ $1.8 R_{\mathrm{E}} \times 9 R_{\mathrm{E}}$ ). The eccentric nature of the orbit means that roughly 9 hours of the 18-hour orbit will be spent at distances greater than $6 R_{\mathrm{E}}$ from the Earth. From these geocentric distances, the field of view required to image the full oval is relatively large $\left(\sim 8^{\circ}\right)$ for conventional reflective imaging instruments. Yet, as we shall discuss in more detail below, it is a requirement also to be able to resolve approximately $0.5 \mathrm{deg}$ in latitude from $9 R_{\mathrm{E}}$, i.e., $\sim 50 \mathrm{~km}$. This places unusual demands on the optical system.

In the anticipated orbit, the instrument will experience a severe radiation environment, a factor which must be taken into consideration in all design areas. The radiation dosage expected on the despun platform over the two year nominal mission lifetime is approximately $275 \mathrm{krads}$. In view of these considerations, the electronics of the instrument have been designed with a significant degree of redundancy.

The instrument designed to meet the above requirements is a reflective system that operates over a wavelength range from $1300 \AA$ to $1900 \AA$, and images onto a 2-dimensional intensified-CCD detector. Details are given below.

\subsection{FIELD OF VIEW/SPATIAL RESOLUTION}

The POLAR spacecraft will be launched into a highly elliptical orbit with apogee at $9 R_{\mathrm{E}}$ and perigee near $1.8 R_{\mathrm{E}}$. The optimum spatial resolution and extent of coverage therefore vary significantly around the $\sim 18$ hour orbit. This 


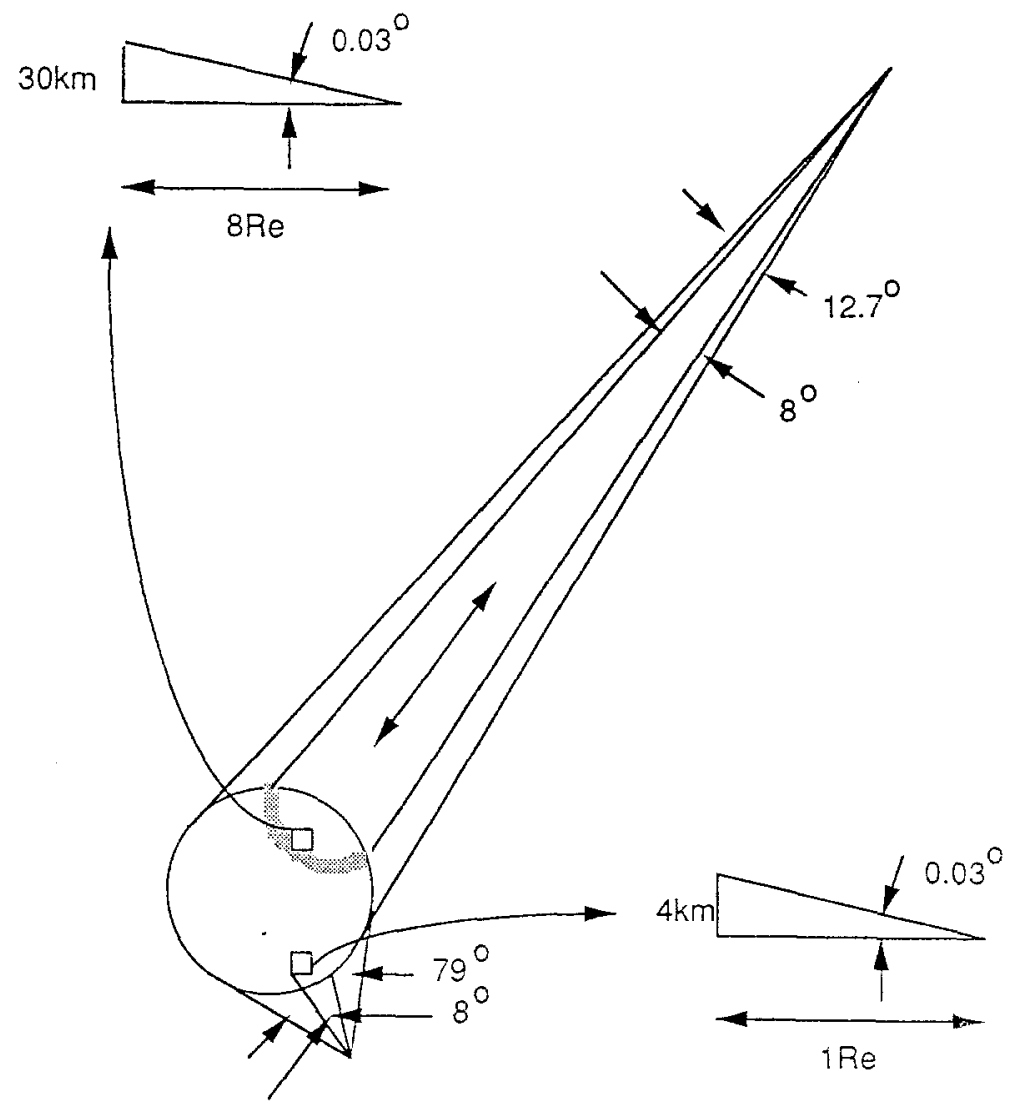

Fig. 2.2.1. Illustration of the field of view of the instrument as projected on the Earth from various points in the orbit.

TABLE 2.2 .1

Distance traveled by auroral feature

\begin{tabular}{lllcc}
\hline & \multicolumn{4}{l}{$\begin{array}{l}\text { Distance traveled }(\mathrm{km}) \text { for various horizontal } \\
\text { auroral velocities }\end{array}$} \\
\cline { 2 - 5 } Integration time, $\mathrm{s}$ & $0.5 \mathrm{~km} \mathrm{~s}^{-1}$ & $1.0 \mathrm{~km} \mathrm{~s}^{-1}$ & $2.0 \mathrm{~km} \mathrm{~s}^{-1}$ & $4.0 \mathrm{~km} \mathrm{~s}^{-1}$ \\
\hline 15 & 7.5 & 15 & 30 & 60 \\
30 & 15 & 30 & 60 & 120 \\
40 & 20 & 40 & 80 & 160 \\
60 & 30 & 60 & 120 & 240 \\
\hline
\end{tabular}

is illustrated in Figure 2.2.1. Choice of field of view becomes a trade-off between spatial resolution and global coverage. The larger the field of view, the more global the coverage, but the spatial resolution is reduced because the instantaneous field of view is imaged onto a fixed number of pixels and, in addition, the optical aberrations increase with increased field of view. 


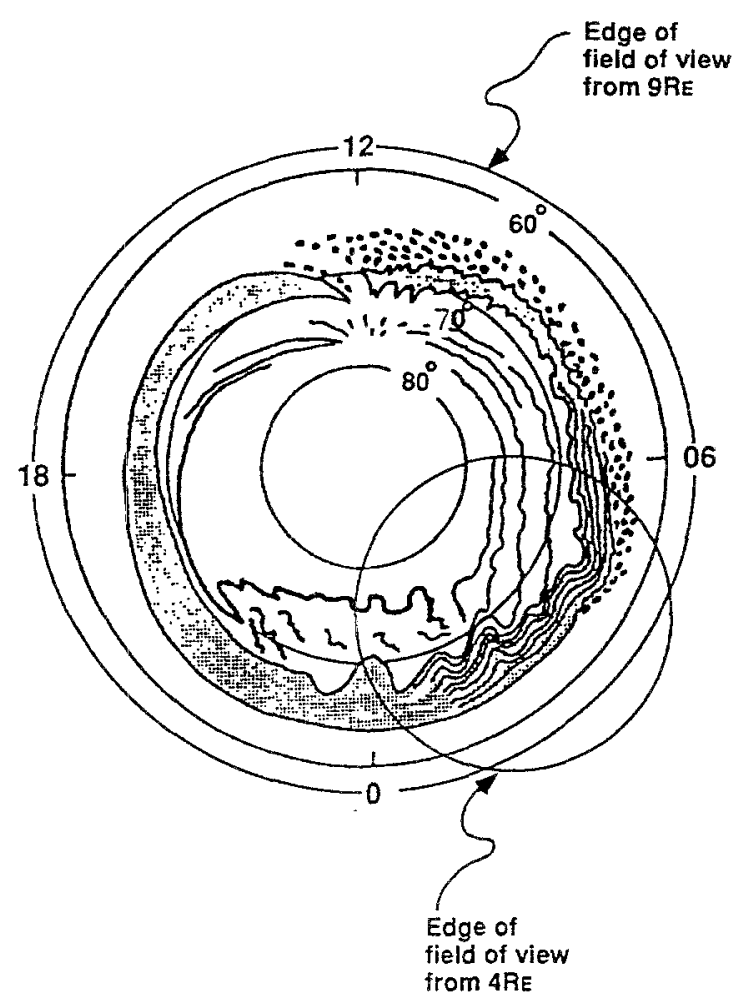

Fig. 2.2.2. Illustration of the $8^{\circ}$ field of view projected on a representative auroral oval (Akasofu, 1981) from an instrument at $9 R_{E}$ and $4 R_{E}$.

TABLE 2.2 .2

Ultraviolet imager spatial resolution/global coverage summary

\begin{tabular}{llll}
\hline $\begin{array}{l}\text { S/C altitude } \\
\left(\mathrm{R}_{\mathrm{E}}\right)\end{array}$ & $\begin{array}{l}8 \mathrm{deg} \text { FOV } \\
200 \times 224 \text { pixels } \\
\left(\mathrm{km} \mathrm{pixel}^{-1}\right)\end{array}$ & $\begin{array}{l}\text { Global coverage } \\
\text { (latitude reached when } \\
\text { covered }\end{array}$ & $\begin{array}{l}\text { Degrees } \\
\text { latitude }\end{array}$ \\
\hline 8 & $39.6 \times 34.7$ & $57^{\circ}$ & $66^{\circ}$ \\
7 & $34.7 \times 30.4$ & $62^{\circ}$ & $56^{\circ}$ \\
6 & $29.7 \times 26.0$ & $66^{\circ}$ & $48^{\circ}$ \\
5 & $24.8 \times 21.7$ & $70^{\circ}$ & $40^{\circ}$ \\
4 & $19.8 \times 17.3$ & $74^{\circ}$ & $32^{\circ}$ \\
3 & $14.8 \times 13.0$ & $78^{\circ}$ & $24^{\circ}$ \\
2 & $9.9 \times 8.7$ & $82^{\circ}$ & $16^{\circ}$ \\
1 & $4.9 \times 4.3$ & $86^{\circ}$ & $8^{\circ}$ \\
\hline
\end{tabular}

Typically, the auroral oval lies poleward of a magnetic latitude of $60^{\circ}$. Because a major objective of the UVI investigation is to obtain global coherent images of the auroral footprint (rather than a montage of smaller images taken on different 
time centers), the UVI has been optimized so that, for typical conditions, the entire oval will be imaged for spacecraft altitudes greater than $6 R_{\mathrm{E}}$ (i.e., $\sim 9$ hours of each orbit). As mentioned above, this requires an $8^{\circ}$ field of view. An $8^{\circ}$ field of view projected on a representative auroral oval from $9 R_{\mathrm{E}}$ is shown in Figure 2.2.2. At lower altitudes, smaller geographic coverage will be simultaneously acquired but with greater spatial resolution. As will be discussed in greater detail in Section 2.6, the CCD array used here has 224 by 200 pixels across the two perpendicular diameters of the $8^{\circ}$ circular field of view. This means that the per pixel spatial resolution is $0.036^{\circ}$ in one direction, and $0.04^{\circ}$ in the other direction. This angular resolution corresponds to $0.62 \times 0.70$ milliradians $^{2}$. This is a higher angular resolution than the accuracy to which the pointing of the despun platform of the spacecraft will be known. It is believed that the despun platform pointing will be stable (jitter) to approximately $0.06^{\circ}$. The actual knowledge of the pointing from the platform is not known this well, however, and will only be provided to approximately $0.2^{\circ}$. The higher angular resolution of the instrument will be used to refine the pointing knowledge by observing stars from time to time during the course of the mission.

An important consideration in deciding on the spatial resolution for an imager is the horizontal speed of the aurora. In many cases of interest the aurora will move with a horizontal speed of 1 to perhaps $5 \mathrm{~km} \mathrm{~s}^{-1}$. In Table 2.2.1 is shown a summary of the distance traveled by an auroral form in the course of an integration period for various horizontal velocities.

Often, the weaker features of interest ( $<250$ Rayleighs, say) will require integration periods in the 30 to 60 -s range in order to acquire images with sufficient signal to noise ratio. An instrument design which emphasizes high spatial resolution at the expense of full field of view, must optimize for auroras that have very low horizontal velocities, are rather bright, and would require greater platform stability than can be provided by the POLAR spacecraft. In addition, we must consider the geometry with which auroral arcs are viewed. Auroral arcs are narrow (about $10 \mathrm{~km}$ wide), but this width can only be observed when looking parallel to the magnetic field. Typically the instrument line of sight will cut through the arc at a slant which will broaden the arc. For these reasons we have decided to maximize the global coverage and design the per pixel field of view to be a factor of 2 better than the ultimate limitation of the spacecraft/despun platform.

In Table 2.2.2 we show the global coverage (in latitude reached from the pole) for an $8^{\circ}$ field of view. From apogee, instantaneous coverage is obtained from the pole to $57^{\circ}$ latitude. Since the despun platform can be stepped in $1^{\circ}$ increments in the plane of the orbit, the $8^{\circ}$ field of view can be offset from the nadir in this plane. As the vehicle proceeds around the orbit moving closer to the Earth, the instantaneous area is reduced but spatial resolution of up to $4 \mathrm{~km}$ is obtained. The spatial resolution given in Table 2.2.2 is shown in the two dimensions of the CCD as the pixels are rectangular. The full field of view is, however, a circular $8^{\circ}$. 


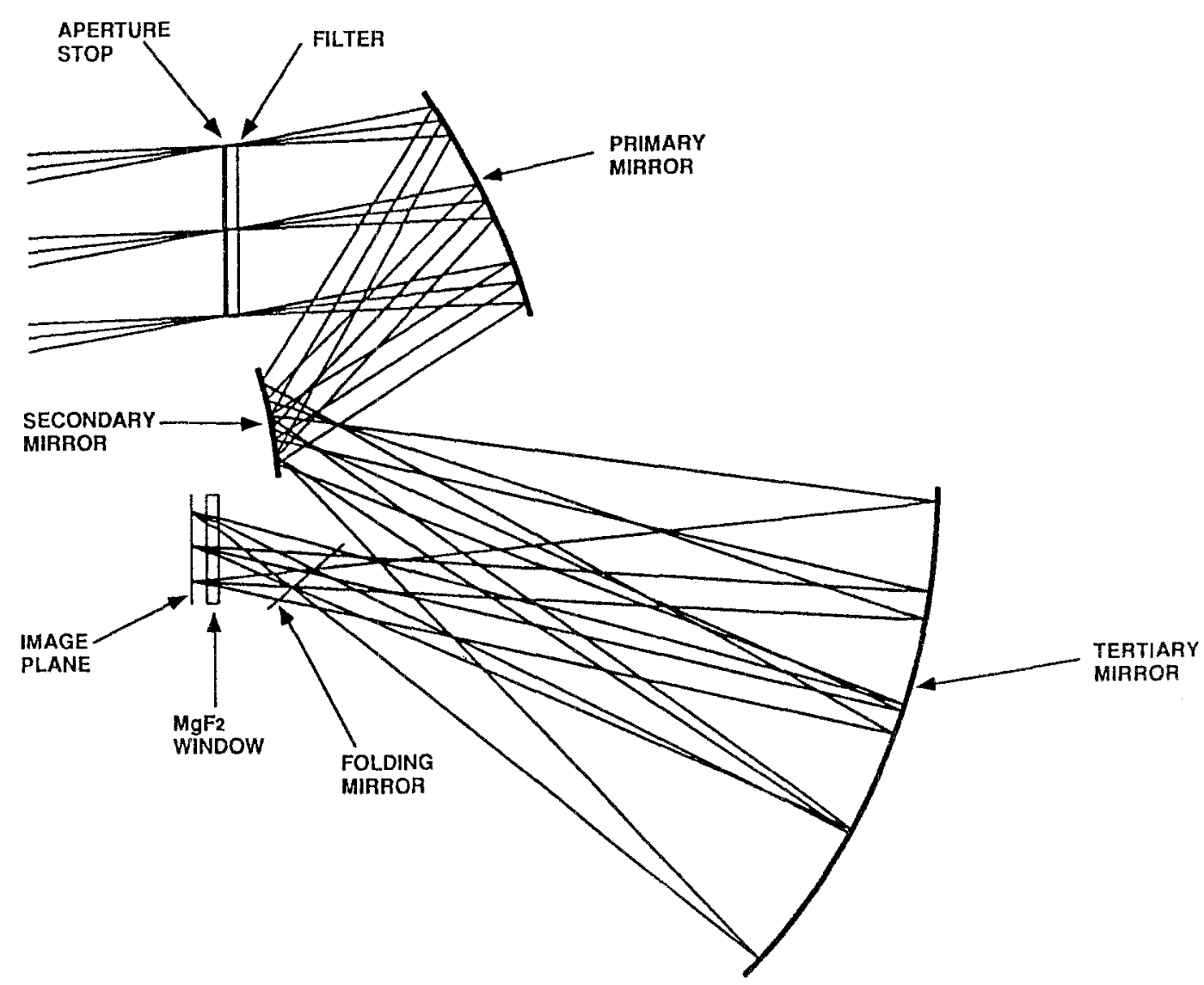

Fig. 2.3.1. The optical configuration of the Ultraviolet Imager.

The auroral view depicted in Figure 2.2.2 is the optimum with the spacecraft at apogee above the magnetic pole and for typical auroral dimensions. The POLAR spacecraft will be placed in an orbit where apogee after launch is a few degrees off the magnetic pole, with a slow precession (in the course of a year or so) across the pole to a few degrees on the other side. As a result, viewing conditions from apogee should be rather good for much of the nominal mission if the desired orbit is achieved. At times, for more oblique views, part of the oval will be over the horizon of the Earth. For very magnetically quiet conditions, the dimensions of the oval will shrink, making imaging of the entire oval easier, but for very disturbed conditions, when, for example, the aurora occur down to mid-latitudes, the despun platform offset capability will have to be used to image portions of the enlarged oval.

As is discussed in Section 2.6, the size of a single pixel at the image plane is $87 \times 74 \mu \mathrm{m}^{2}$. As will be discussed in the section on the optical system below, the blur spot size is smaller than a single pixel over most of the image plane, with 

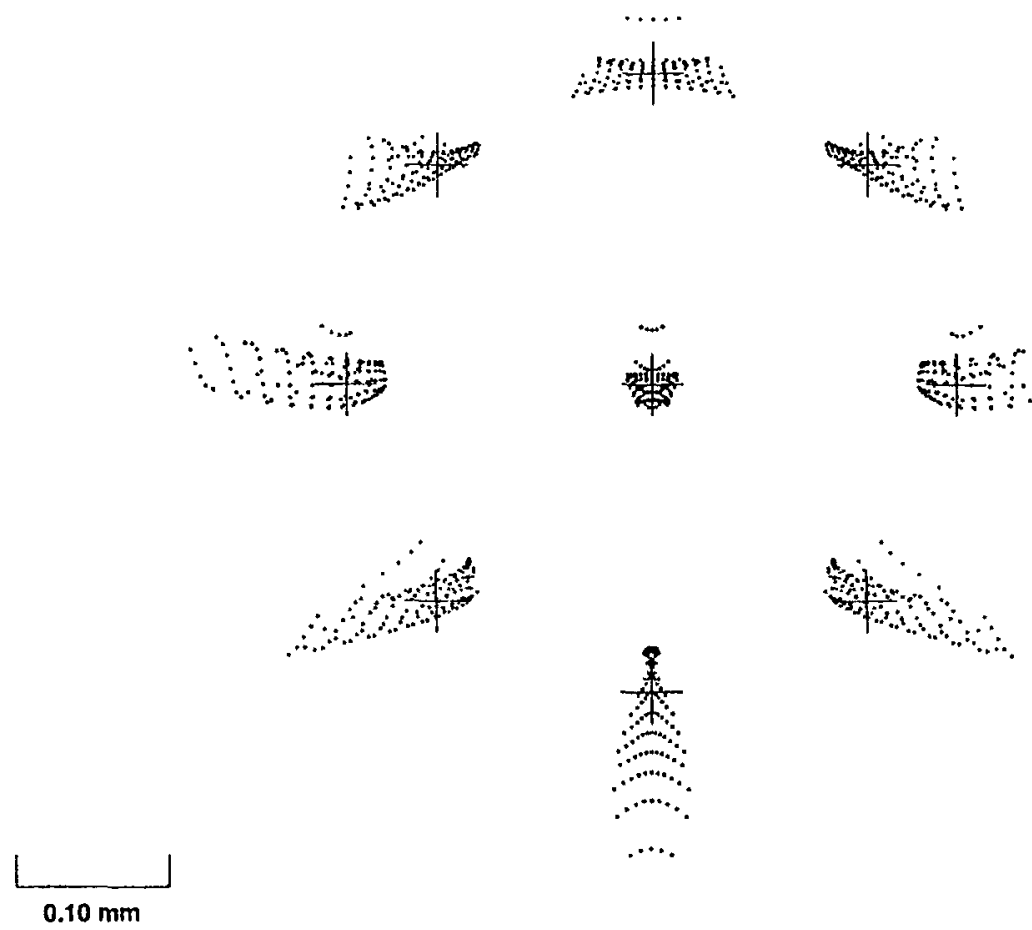

Fig. 2.3.2. Blur spot diagrams for the optical configuration shown in Figure 2.3.1. The spot patterns are shown for the center and around the edge of the $8^{\circ}$ field of view.

TABLE 2.3.1

Mirror r.m.s. roughness

\begin{tabular}{ll}
\hline Mirror & Roughness, A \\
\hline Primary & $<20$ \\
Secondary & $<15$ \\
Tertiary & $<20$ \\
\hline
\end{tabular}

the exception of the field of view extremes at the shortest wavelength $(1304 \AA)$, where it reaches two pixels. However, the emission at $1304 \AA$ is optically thick and naturally smeared, so there is no loss of geophysical information.

\subsection{THE OPTICAL SYSTEM}

The optical configuration selected for the UVI instrument is shown in Figure 2.3.1. This configuration is the outcome of a study involving a number of alternative designs and was chosen because of its impressive speed $(f / 2.9)$ combined with good imaging over the full $8^{\circ}$ field of view. This design, which is a development of the anastigmatic three-mirror designs reported by Korsch (1975, 

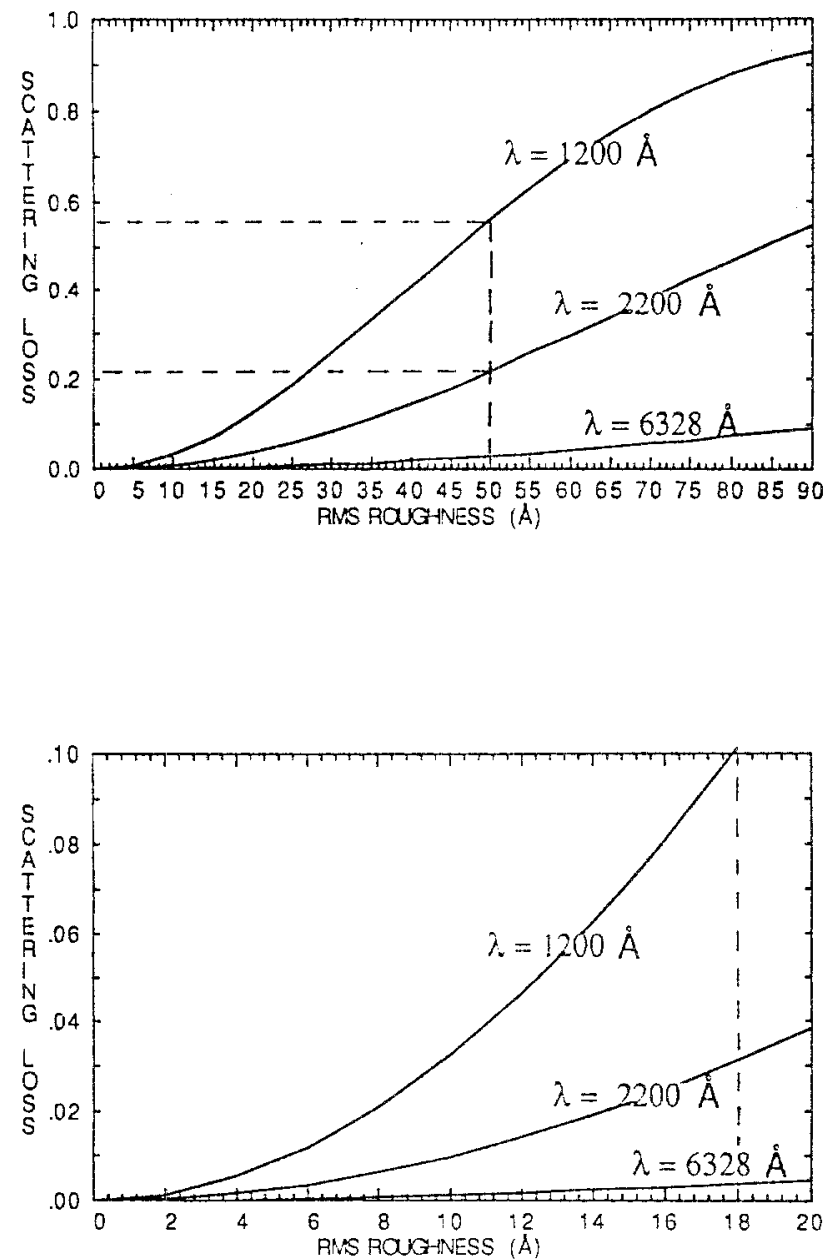

Fig. 2.3.3. Scattered light loss as a function of mirror surface roughness and wavelength (Harvey, private communication, 1989).

1977, 1980) and Cook (1987), provides an unobscured optical aperture, excellent baffling, flat field, provision for optical filter insertion and general compactness. The design (Johnson, 1988) comprises three reflective components which provide color-corrected performance over the wavelength region of interest. The primary, secondary and tertiary mirrors share a common optical axis, a significant factor in ease of alignment. Each mirror is a portion of a conic section. The primary and secondary mirrors form an intermediate image. By locating the entrance pupil eccentrically with the optical axis of the system, the intermediate image surface is accessible for the purpose of locating a field stop and associated baffles to negate scattered light and improve the off-axis rejection performance of the system. The tertiary mirror is used to relay the intermediate image to the final image plane. 


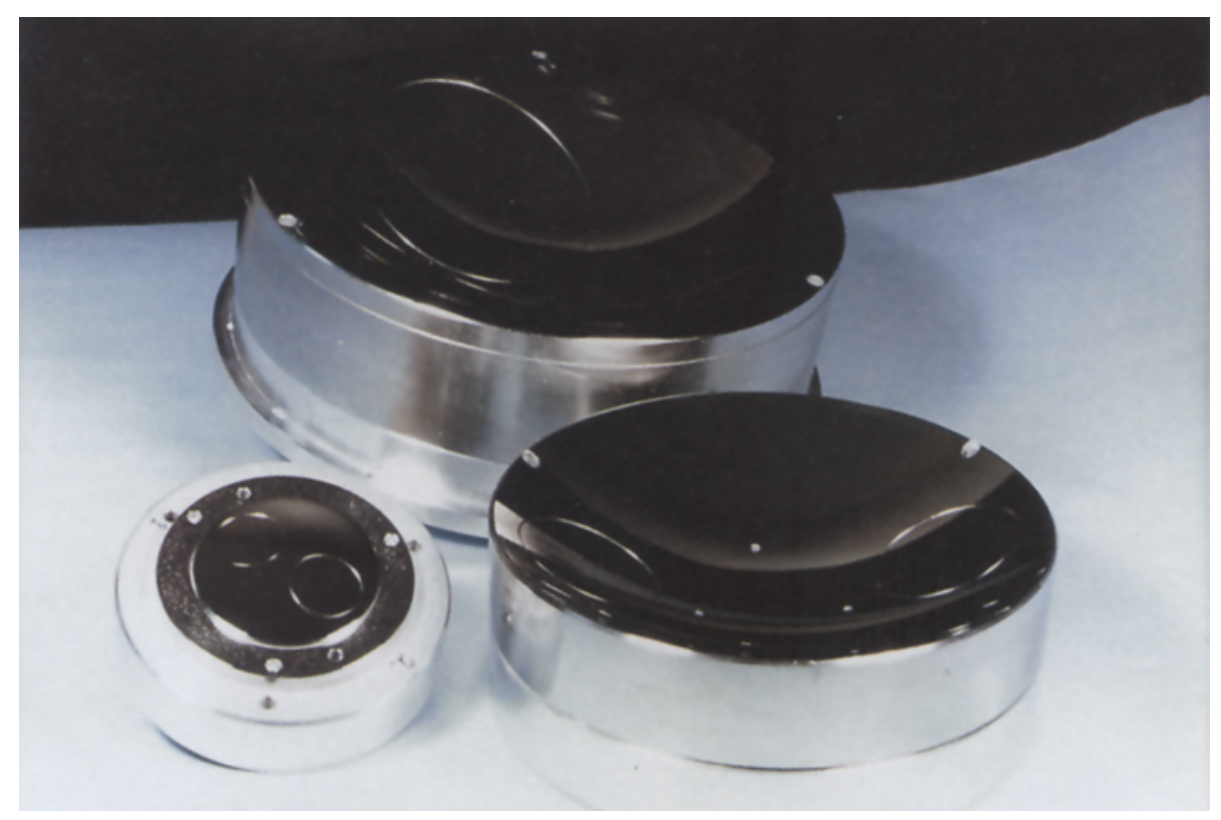

Fig. 2.3.4a. Photograph of the three prototype mirrors used in the ultraviolet imager engineering model.

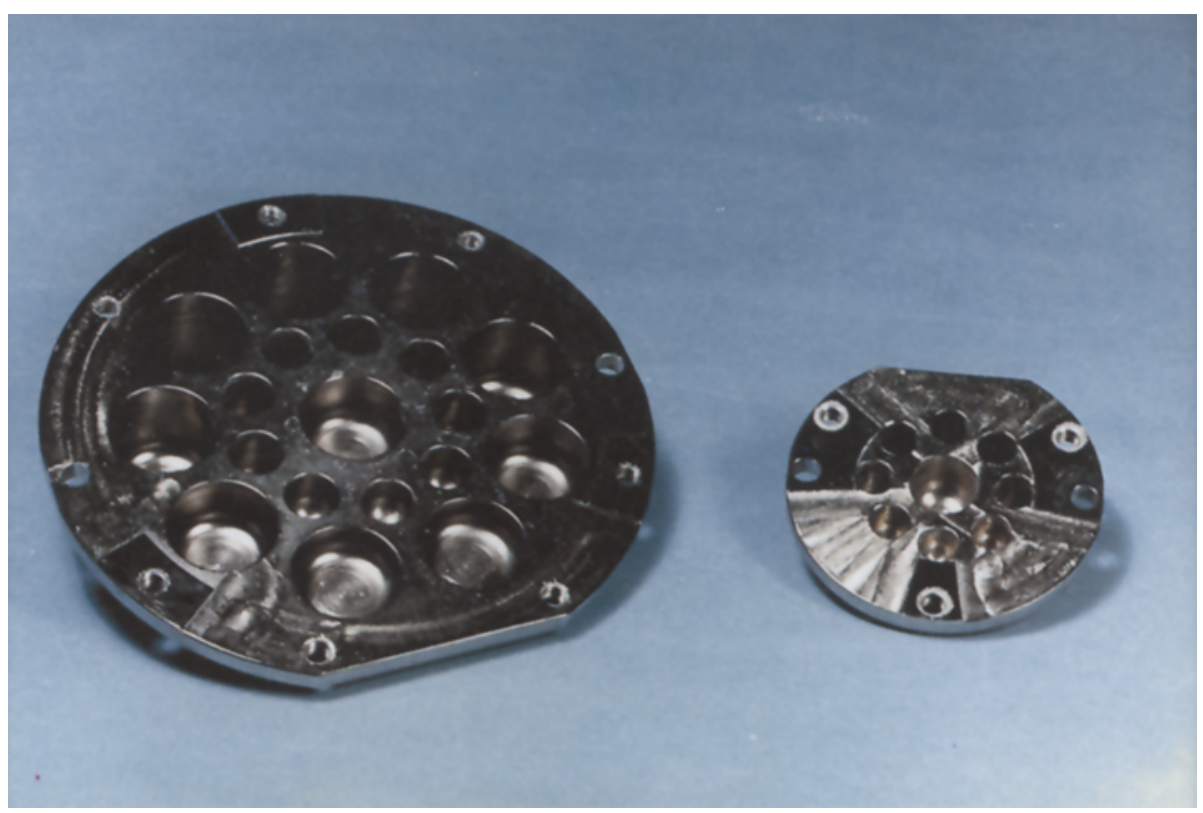

Fig. 2.3.4b. Showing the weight relief in the back of two of the mirrors. 
Both the primary and tertiary mirrors are ellipses, while the secondary mirror is an hyperbola. Each of these mirrors have sixth, eighth, and tenth order aspheric deformations. The primary and tertiary mirrors have very modest but important deformations, while the secondary mirror has comparatively strong, high order aspheric deformations. There are no tilts or decenters of these components with respect to their common axis. The center of the aperture stop is displaced from the optical axis and is tilted by $6.0 \mathrm{deg}$.

Early in the program, a proof of concept instrument was built and tested, and this is reported elsewhere (Johnson, 1988) together with a detailed discussion of the aberrations of the system. The system parameters were subsequently fine-tuned to minimize encircled energy variations across the field of view and to take into consideration the $\mathrm{MgF}_{2}$ window on the image intensifier. Spot diagrams for the system are shown in Figure 2.3.2. This figure shows a pictorial representation of the image surface, with the outer circle representing the $8^{\circ}$ diameter field of view. The center of this diagram is the center of the active field of view and is located at $-6.0^{\circ}$ from the common mirror axis in the total system field of view. Due to the nature of the system, the images are symmetric about the elevation axis. The largest reduction in optical quality is introduced by the $\mathrm{MgF}_{2}$ faceplate of the image intensifier. This faceplate is $3.0 \mathrm{~mm}$ thick (which is as thin as we felt was viable) and introduces chromatic aberrations which are the worst at the shortest wavelengths. At the image plane, a single picture element (pixel) is $0.074 \times 0.087 \mathrm{~mm}^{2}$. At $1304 \AA$ the blur spot size reaches a worst case of two pixels at the edges of the image plane. At all longer wavelengths, the blur spot sizes are comparable with or less than a single pixel, with $80 \%$ of the encircled energy well within a single pixel. The image surface is essentially flat over the $8^{\circ}$ diameter of the field of view (an $18 \mathrm{~mm}$ diameter image plane).

The image quality is achieved through careful design and fabrication of the three aspheric reflective surfaces. As was mentioned in Section 2.1, auroral images can exhibit a large in-field dynamic range. This, together with the spatial resolution requirements, drives the specifications for the surface roughness of the mirrors. The issues are illustrated in Figure 2.3.3 which shows the percentage light scattered from a point source as a function of surface r.m.s. roughness and wavelength. Our objective was to keep scattered light loss to less than $10 \%$, which requires that the mirror surface be smoother than $20 \AA$ r.m.s. at our shortest wavelength. The mirrors are fabricated from aluminum blanks which are then diamond turned, nickel plated, diamond turned again, and finally polished (Figure 2.3.4) and then coated. In parallel with this effort, null reflectors to test the surface figure were designed and fabricated, and a new technique was developed to measure the surface roughness for such large aspheric surfaces. This work was done by the University of Alabama in Huntsville. The r.m.s. roughness achieved for each is shown in Table 2.3.1. In designing and fabricating mirrors for use in the FUV, particular attention must be paid to both the microroughness and the mid spatial frequency surface roughness, as both of these fabrication limitations result in the scattering of 


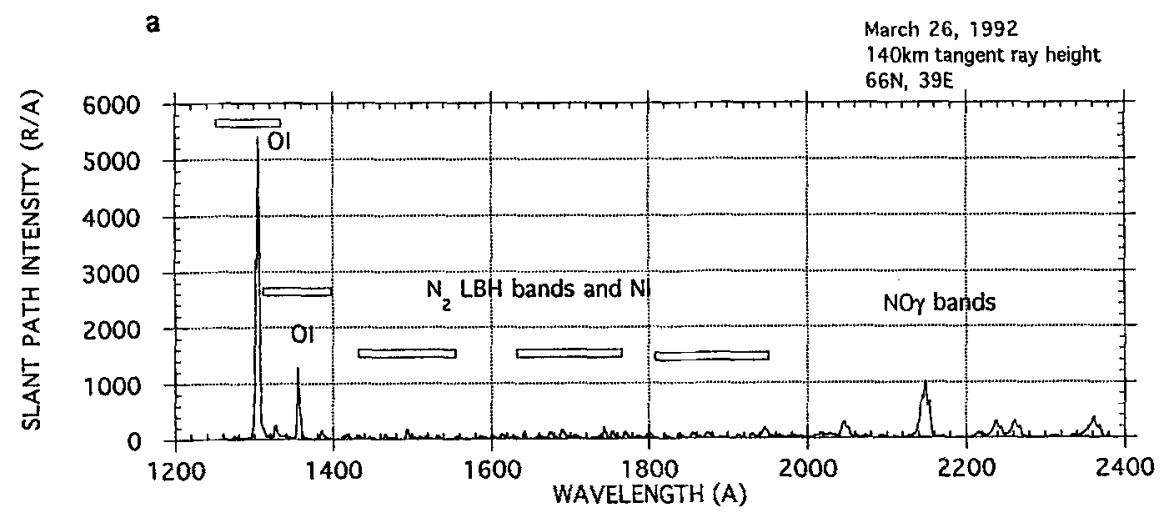

Fig. 2.4.1a. High latitude vacuum ultraviolet spectrum of the dayglow, showing the principal dayglow/dayside auroral features in this wavelength range. This spectrum was obtained for a $140 \mathrm{~km}$ tangent ray height and is representative of a soft aurora. Also shown in the figure are the bandpasses of the 5 filters used in the UVI instrument: (a) shows the spectrum full scale to illustrate the relative magnitudes of the features that need to be measured and those that need to be rejected.

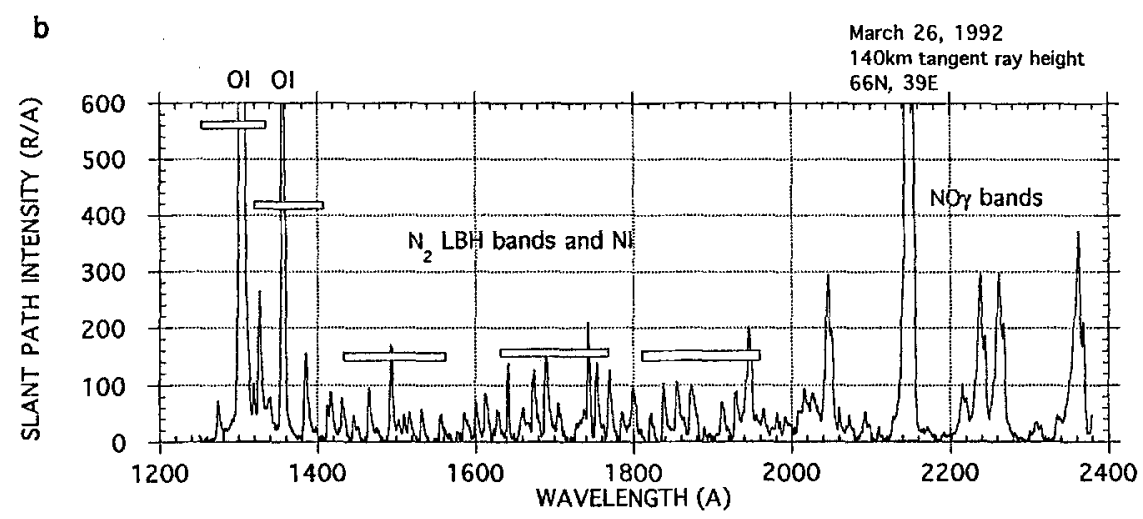

Fig. 2.4.1b. High latitude vacuum ultraviolet spectrum of the dayglow, showing the principal dayglow/dayside auroral features in this wavelength range. This spectrum was obtained for a $140 \mathrm{~km}$ tangent ray height and is representative of a soft aurora. Also shown in the figure are the bandpasses of the 5 filters used in the UVI instrument: (b) is plotted on a different scale to show the weaker features.

energy. The optical configuration and internal baffling, together with high mirror surface quality, results in very low scattered light at the detector.

The optical elements require precision alignment, which places demands on the mechanical structure described in a later section.

\subsection{FUV FILTERS}

In order to measure the key vacuum ultraviolet diagnostic emission features, the instrument operates from $1300 \AA$ to $1900 \AA$. The features are separated using specially designed interference filters which are summarized in Table 2.4.1. 


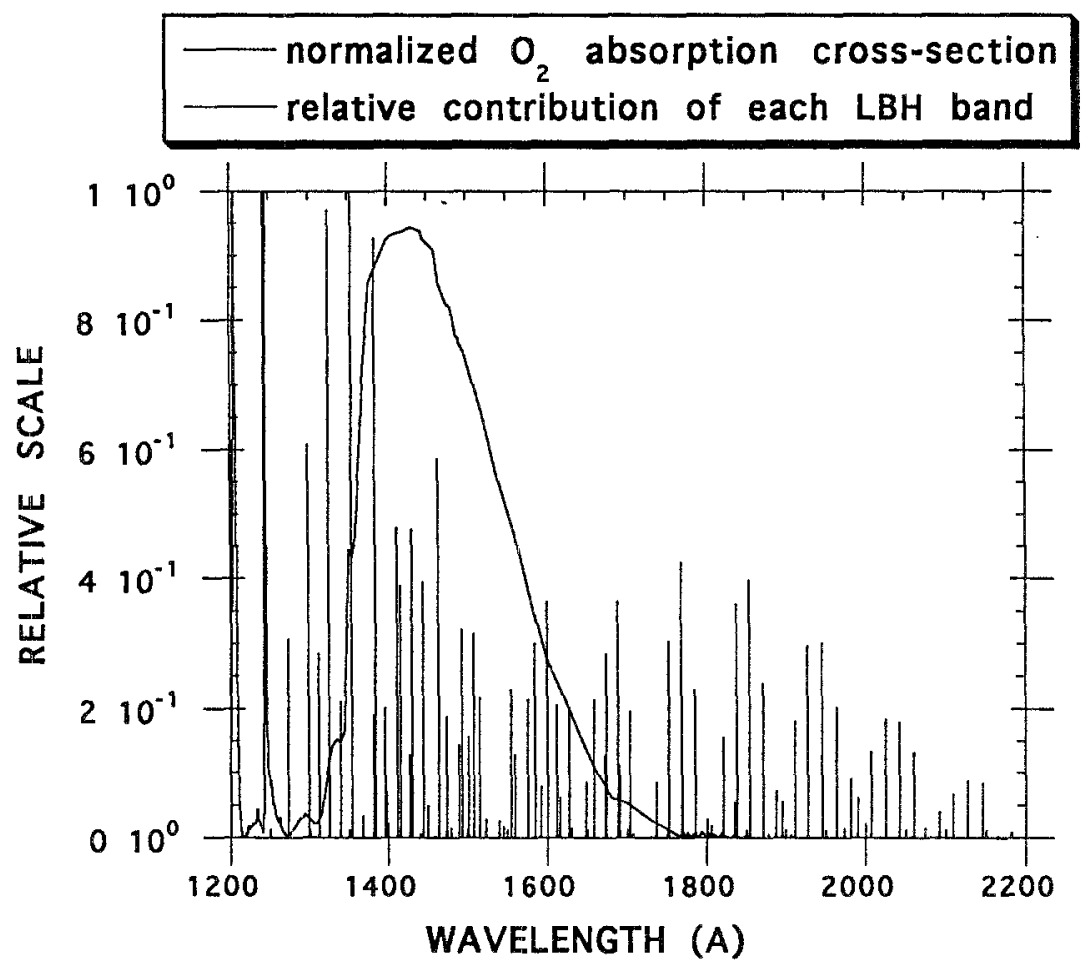

Fig. 2.4.1c. $\mathrm{O}_{2}$ absorption cross-section (in arbitrary units). The lines show the percentage contribution of each LBH band to the total LBH emission.

TABLE 2.4.1

Feature selection

\begin{tabular}{rlll}
\hline$\lambda(\AA)$ & Feature & Purpose & $\Delta \lambda(\AA)$ \\
\hline 1304 & O I & atomic oxygen & 30 \\
1356 & $\mathrm{O}_{\mathrm{I}}$ & characteristic energy, $\mathrm{O}_{2}$ & 50 \\
$\sim 1500$ & $\mathrm{~N}_{2} \mathrm{LBH} / \mathrm{N} \mathrm{I}$ & characteristic energy, $\mathrm{O}_{2}$ & 80 \\
$\sim 1700$ & $\mathrm{~N}_{2} \mathrm{LBH} / \mathrm{NI}_{\mathrm{I}}$ & total energy & 90 \\
$\sim 1900$ & scattered sunlight & contaminant & 100 \\
\hline
\end{tabular}

The quantitative performance of the instrument depends to a large degree on the quality of these interference filters. Filters generally available at the present time for this wavelength region are rather broadband ( $200 \AA$ to $400 \AA$ ). Figure 2.4.1(a) shows the principal emission features of the aurora in this wavelength range. This figure shows data taken with the Imaging Spectrometric Observatory flown on the ATLAS-1 shuttle mission (Torr et al., 1994), with the field of view of the instrument looking at a tangent ray height of $140 \mathrm{~km}$. Because of the viewing geometry, it 
TABLE 2.4 .2

Ultraviolet imager filter summary

\begin{tabular}{llllll}
\hline Parameters & $1304 \AA$ & $1356 \AA$ & LBH-short & LBH-long & SOLSPEC \\
\hline Transmission filter: & $\mathrm{T} 1304$ & $\mathrm{~T} 1356$ & $\mathrm{TLBH}$-short & TLBH-long & TSolar \\
Substrate & $\mathrm{MgF}_{2}$ & $\mathrm{MgF}$ & $\mathrm{BaF}_{2}$ & Fused silica & Fused silica \\
Number of layers & 35 & 2 & 1 & - & 19 \\
Film materials & $\mathrm{LaF}_{3}, \mathrm{MgF}_{2}$ & $\mathrm{MgF}_{2} / \mathrm{BaF}_{2}$ & $\mathrm{MgF}_{2}$ & - & $\mathrm{Al}_{2} \mathrm{O}_{3} / \mathrm{MgF}_{2}$ \\
Reflection filters: & $\mathrm{R} 1304$ & $\mathrm{R} 1356$ & $\mathrm{RLBH}-$ short & $\mathrm{RLBH}-$ long & Rsolar \\
Substrate & Pyrex & Pyrex & Pyrex & Pyrex & Pyrex \\
Number of layers & 35 & 35 & 35 & 35 & 2 \\
Film materials & $\mathrm{MgF}_{2} / \mathrm{LaF}_{3}$ & $\mathrm{MgF}_{2} / \mathrm{LaF}_{3}$ & $\mathrm{MgF}_{2} / \mathrm{LaF}_{3}$ & $\mathrm{MgF}_{2} / \mathrm{LaF}_{3}$ & $\mathrm{AlMgF}_{2}$ \\
\hline
\end{tabular}

\section{VUV AURORA and SCATTERED SUNLIGHT}

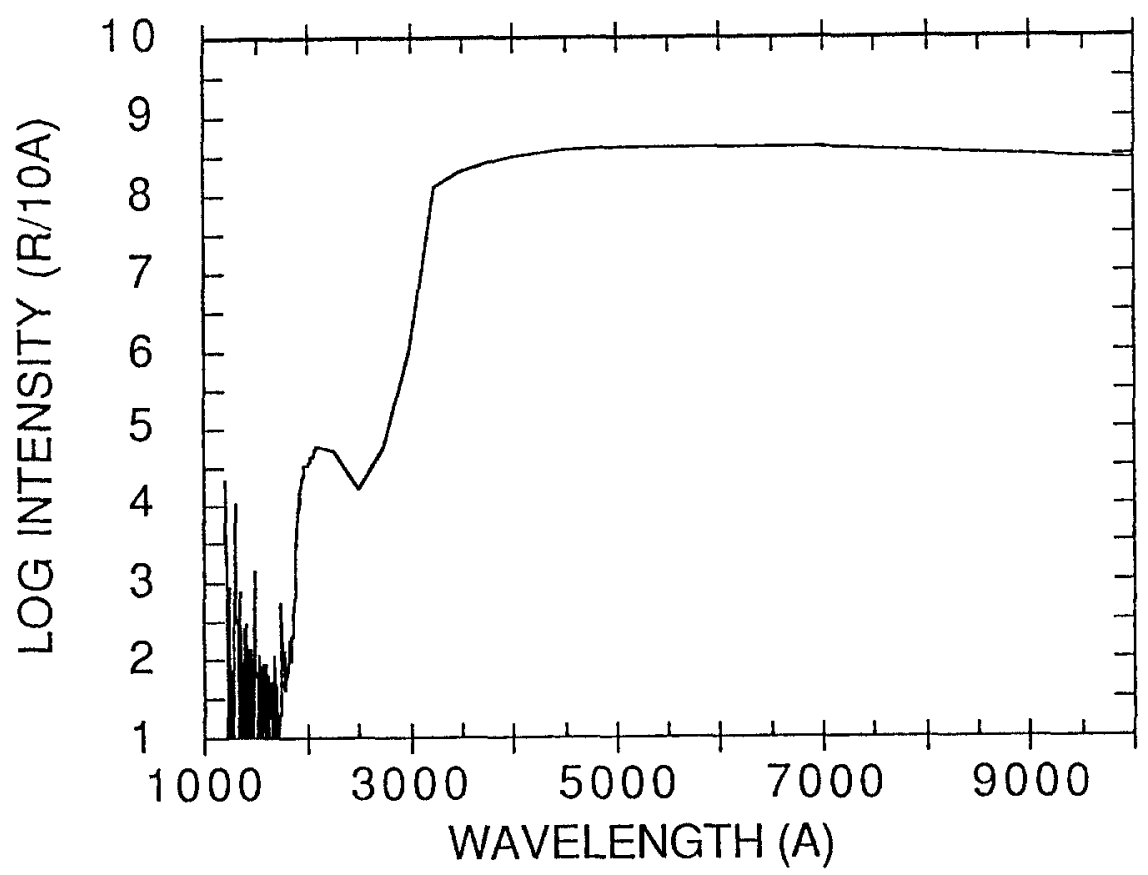

Fig. 2.4.2. Brightness seen by nadir viewing instrument looking at the sunlit Earth (as a function of wavelength) with the FUV auroral features superimposed. This figure is a composite of the following: (a) 1200-2000 $\AA$ - our synthetic auroral spectrum; (b) 2000-4000 $\mathrm{A}$ - from Barth (1966); (c) 4000-10000 $\AA$ - derived from the solar spectral distribution of Allen (1973). 


\section{Pre-UVI Filters}

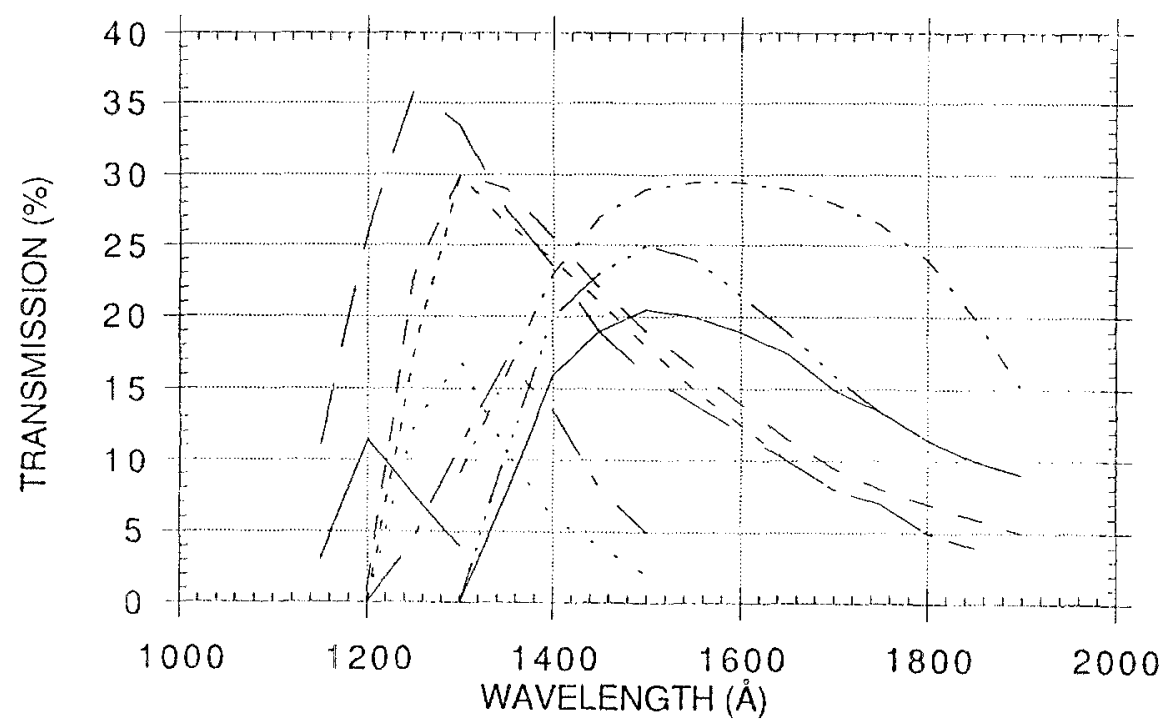

Fig. 2.4.3. Illustration of conventional FUV filters available prior to those developed for the ultraviolet imager.

does not show the scattered sunlight, or albedo, component that is present in nadir looking data. For more detail of the features in this spectrum see Torr et al. (1994c). Most important to our diagnostic purposes are the O I multiplet at $1356 \AA$ and the $\mathrm{N}_{2}$ Lyman-Birge-Hopfield bands. The latter must be measured in two segments: one at shorter wavelengths (LBH-short) where there is strong $\mathrm{O}_{2}$ absorption in the Schumann Runge continuum, and one at longer wavelengths (LBH-long) where the $\mathrm{O}_{2}$ absorption is not significant. The $\mathrm{O}_{2}$ absorption cross-section is shown in Figure 2.4.1(c) from which it can be seen that the absorption is strongest near $1400-1500 \AA$. We have therefore designed the LBH-short filter to peak at $1500 \AA$, and the LBH-long filter to peak at $1700 \AA$ where the absorption is very weak. It can be seen from Figure 2.4.1(a) that at somewhat shorter wavelengths, there is a very much brighter feature: O I $1304 \AA$. The comparable feature, $\mathrm{H} \mathrm{L} \alpha$ at $1216 \AA$, is not shown in this example. Also present at the longer wavelengths are the NO bands and the scattered sunlight continuum (Figure 2.4.2). The FUV auroral features below $2000 \AA$ are superimposed for comparison on the earth albedo spectrum in Figure 2.4.2. The FUV component of the scattered sunlight $(\lambda$ $<3000 \AA$ ), while very weak compared with the intensity at longer wavelengths, is still significant with respect to weak emissions because of its broad wavelength extent in a region where it is very difficult to achieve adequate blocking. It must therefore be dealt with. In addition, the filters must adequately suppress the long wavelength (visible) scattered sunlight. No spectrally pure FUV auroral images of key diagnostic features (O I $1356 \AA$, LBH-short, LBH-long) against the full 


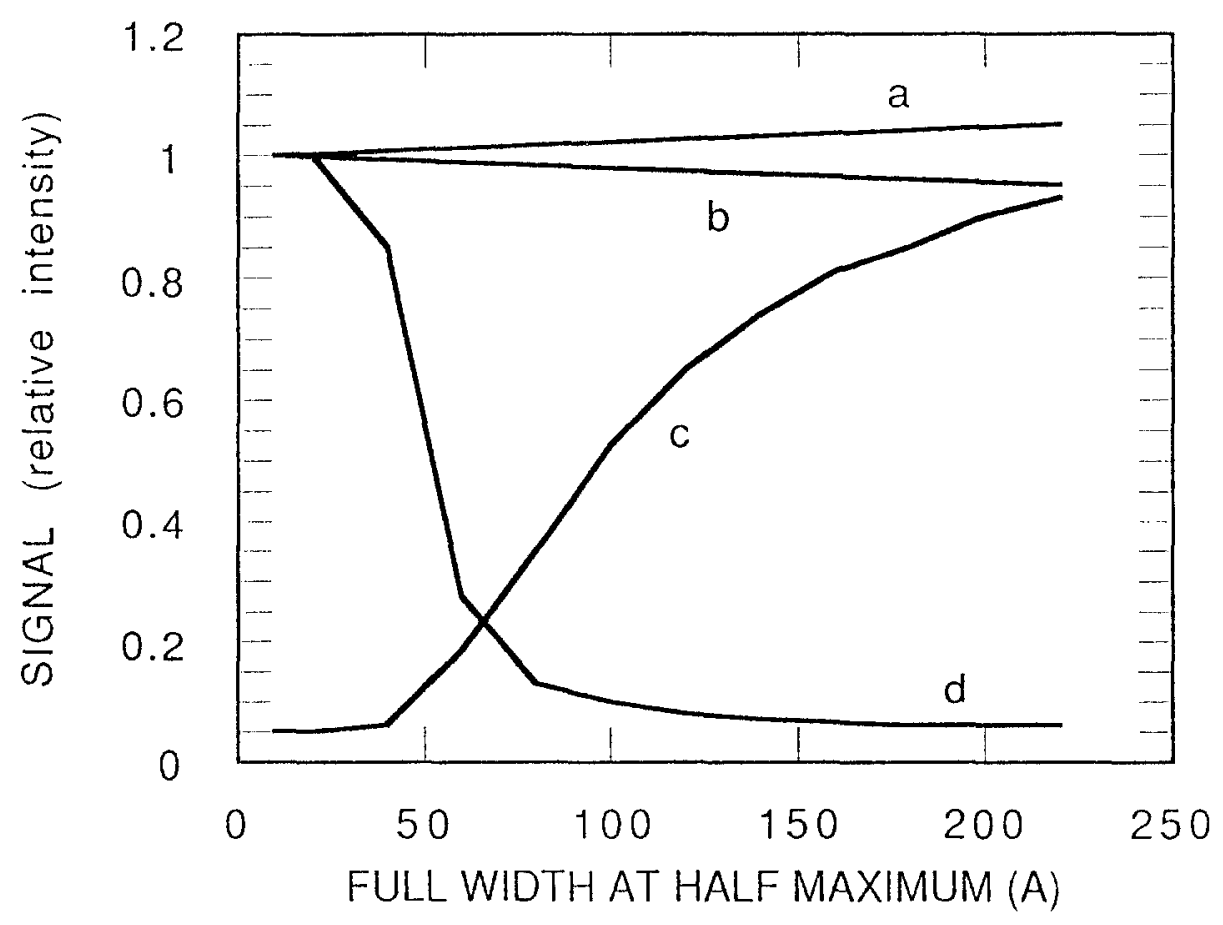

Fig. 2.4.4. Illustration of degradation of measurement with increasing filter bandpass. (a) Total signal passed by filter centered on $1304 \AA$ (intensity of $1304 \AA$ feature is 20 times the feature at $1356 \AA$ ). (b) Component of signal passed by filter that is $1304 \AA$ feature. (c) Total signal passed by filter centered on $1356 \AA$. (d) Component of signal passed by filter that is $1356 \AA$ feature.

sunlit Earth have been achieved previously because of visible sunlight leakage, or contamination by neighboring features. A compilation of the bandpasses of a number of conventionally available FUV filters is shown in Figure 2.4.3. It can be seen that the bandpasses are very broad and ill-defined. As a result, 'contaminant' features, rather than the desired features, have dominated the measurements made with conventional FUV filters. The concept can be simply illustrated as follows: The two oxygen emissions at 1304 and $1356 \AA$ are separated by $52 \AA$. If we assume that these are the only two features present, and that the intensity of the $1304 \AA$ feature is 20 times brighter than the feature at $1356 \AA$, we can calculate the total signal passed by a gaussian filter centered on each of these two wavelengths, as a function of the filter FWHM. The results are shown in Figure 2.4.4. While the impact on a bright feature is not so severe, the figure shows that a filter centered at $1356 \AA$ with a FWHM of $100 \AA$ will yield a signal whose contribution due to the $1356 \AA$ emission line is less than $15 \%$. In the real situation in which there is also $\mathrm{H} \mathrm{L} \alpha$ and scattered sunlight, the results obtained with these filters are much worse. If we are to do quantitative analysis with FUV auroral images, the filters 


\section{UVI FLIGHT INTENSIFIERS}

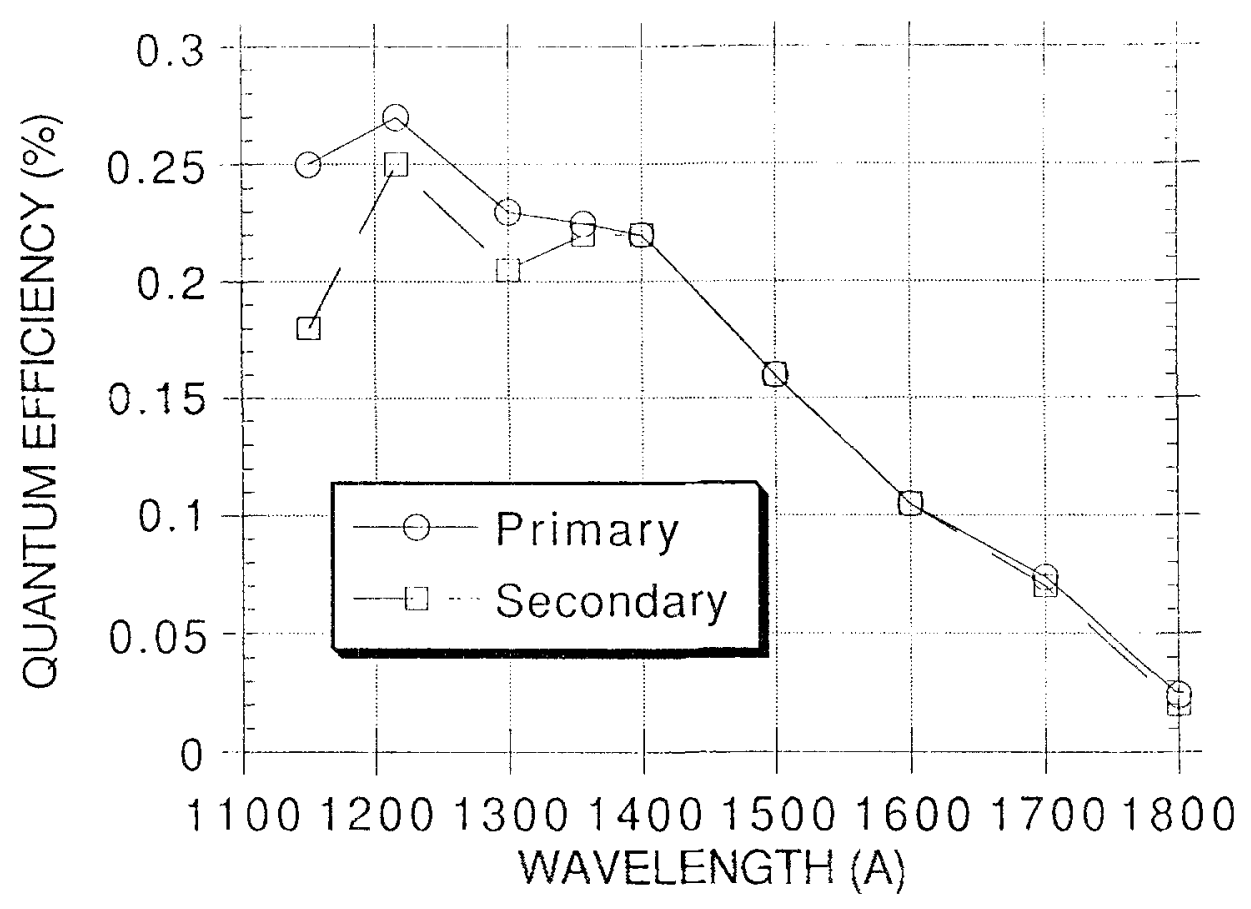

Fig. 2.4.5. Quantum efficiency of the CsI photocathode used in the detector system of the UVI.

must reject contaminant emissions to a level where their noise contribution to the signal-to-noise ratio does not impact the measurement significantly.

Let us consider the visible light rejection requirements. The brightness seen by an instrument looking at the sunlit Earth as a function of wavelength is illustrated in Figure 2.4.2. The sunlit Earth at $\sim 5000 \AA$ is $5 \times 10^{7} R / A$. This is a continuum that extends from the near-UV to the near-IR. The total signal due to scattered visible and IR sunlight is approximately $10^{11} R$. We wish to measure auroral signals as low as $\sim 100 R$. To achieve our desired threshold of detection, this scattered sunlight background level must be reduced to below $100 R$. In other words, the long wavelength continuum must be reduced by a factor of $10^{9}$. In the past, visible blocking was achieved by depositing a thin layer of aluminum on the filters. However, this produced the undesirable effect of large loss in throughput at the FUV wavelengths also, making measurement of the weaker auroral features impossible.

We have conducted a successful development effort to build filters with the required characteristics, allowing adequate separation of the features, accurate subtraction of unwanted contributions in some cases, and adequate suppression of the visible scattered sunlight. 


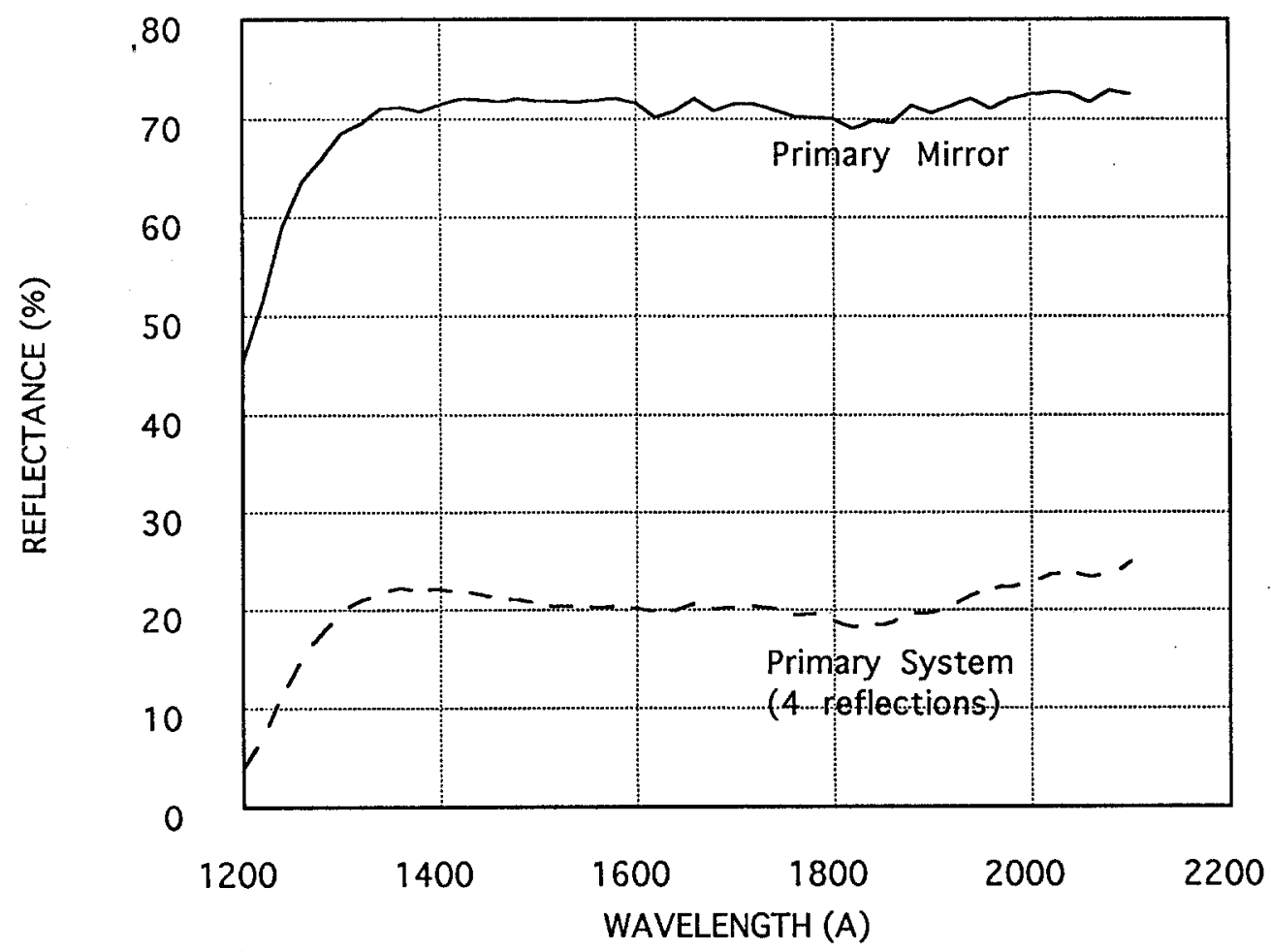

Fig. 2.4.6. Reflectivity of the $\mathrm{Al} / \mathrm{MgF}_{2}$ coated mirrors used in the UVI.

As a first step in the program, a careful survey was made of materials that would be likely candidates for use in such filters (Zukic et al., 1990a). This was followed by a test program to build multilayer transmission filters intended to be forerunners of those needed here (Zukic et al., 1990b). The basic design of the filters used in this instrument (Torr et al., 1993; Zukic et al., 1993) are a combination of reflective and transmissive elements, as summarized in Table 2.4.2. An important additional element in the rejection of long wavelength light, is the use of a 'solar blind' CsI photocathode. In principle, the work function of the CsI material is such that near UV and visible photons are incapable of ejecting photoelectrons. However, in practice, the realities of the manufacturing process are such that it cannot be ensured that traces of other materials are not present, and these may respond to longer wavelength photons. For many applications the contribution from such out-of-band photons would be negligible. However, in dayside FUV auroral imaging, which must be done against the background of the fully sunlit earth, this longer wavelength signal (as discussed above) can be at least 9 orders of magnitude brighter than the FUV signal of interest. Almost no quantum efficiency measurements on 'solar blind' intensifiers have been made over the longer wavelength range of interest. The few measurements that have been made indicate great variability from one device to another, and that one cannot rely 
TABLE 2.4.3

Signal-to-noise ratio for measurement of features in a typical $1 \mathrm{erg} \mathrm{cm}^{-2} \mathrm{~s}^{-1}$ aurora

\begin{tabular}{ll}
\hline Wavelength, $\AA$ & $\begin{array}{l}\text { Signal-to-noise of measurement } \\
\text { for } 1 \mathrm{erg} \mathrm{cm} \mathrm{cm}^{-2} \mathrm{~s}^{-1} \text { night aurora }\end{array}$ \\
\hline 1304 & 49.9 \\
1356 & 10.7 \\
LBH (short) & 17.3 \\
LBH (long) & 13.4 \\
\hline
\end{tabular}

on the CsI photocathode to provide more than $10^{-5}$ long wavelength blocking. In addition, the metallic layers deposited on the inside surface of the intensifier faceplate (so that the faceplate can be voltage biased) effectively act as longer wavelength photocathodes. Adequate blocking must therefore be provided by the filters. The response of the CsI photocathodes used in this instrument are shown in Figure 2.4.5 over the FUV wavelength range. These selected tubes have much better than $10^{-5}$ rejection at the longer wavelengths. We have measured $1.4 \times 10^{-6}$ at $2540 \AA$ and $1.8 \times 10^{-9}$ at $6328 \AA$.

In addition to the filters themselves, which are described below, the mirrors in the instrument are coated with aluminum and $\mathrm{MgF}_{2}$, the reflectivity of which is wavelength dependent. The reflectivity of the $\mathrm{Al} / \mathrm{MgF}_{2}$ coatings for the primary mirror (a single reflecting surface) and for the primary optical system (a total of four reflections -3 aspherics and a folding mirror) is shown in Figure 2.4.6.

Each of the filters used here consists of a reflective and a transmissive component, with the reflective component involving three reflections.

In the case of the $1356 \AA$ filter, for example, the two components (reflective and transmissive) are shown in Figure 2.4.7(a). The reflective element does not have a sufficiently sharp cutoff at the short wavelengths (this is provided by the transmissive element), but has sufficient suppression at the longer FUV wavelengths. By using three of the multilayer thin film reflective elements in series, the long wavelength scattered sunlight is suppressed to $10^{-4}$. The additional $10^{-5}$ (or more) is provided by the CsI photocathode response. So by using these elements in series, their combined effective transmission meets the severe requirements for this measurement. These combine with the mirror coatings and the detector response to give the contribution function shown in Figure 2.4.7(b). To compute this contribution function, we have generated a synthetic spectrum for typical auroral intensities, with a scattered sunlight contribution at the longer wavelengths. The intensities have been binned into $10 \mathrm{~A}$ intervals and convolved with the instrument response function. For a typical aurora, $90 \%$ of what is passed by the filter is the desired $1356 \AA$ signal. The signal to noise ratios for the measurement for an auroral energy flux of $1 \mathrm{erg} \mathrm{cm}^{-2} \mathrm{~s}^{-1}$ (relatively weak) are given in Table 2.4.3. These are 
1356 FILTER

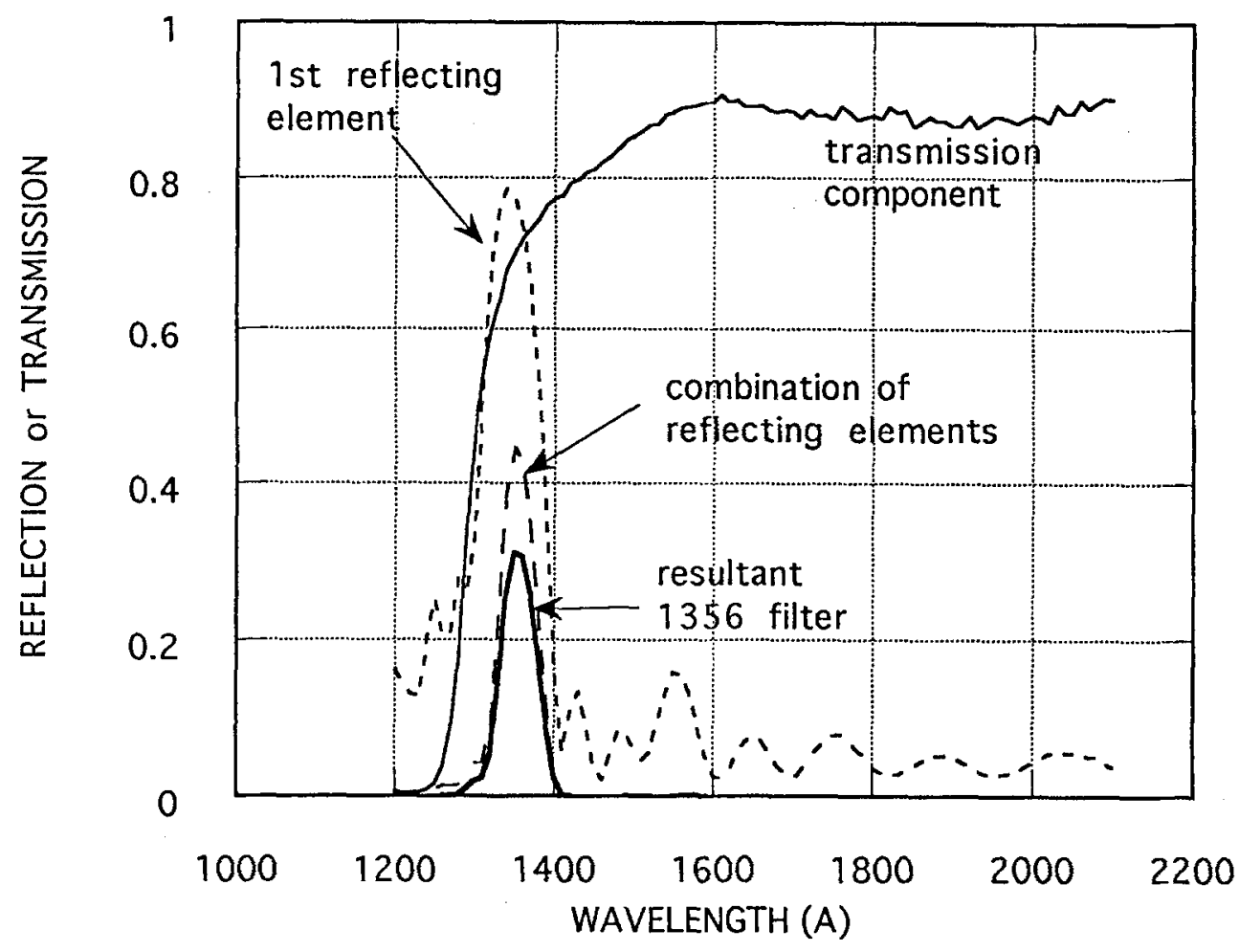

Fig. 2.4.7a. Response of the reflective (solid) and transmissive (dashed) components of the filter elements used for measuring the OI line at 1356A, and of their combination (cross hatched).

sufficiently large to allow the components to be accurately separated by Fourier decomposition.

The same design approach of using three reflective and a transmissive filter in series is used for each of the features. The 5 composite filters are built into cells and are mounted on a filter wheel so that each can be moved into the optical path as required.

The $1304 \AA$ filter components are shown in Figure 2,4.8(a) and the contribution function is shown in Figure 2.4.8(b). Typically, 98\% of the emission passed by the filter is the $1304 \AA$ signal. The usual principal contaminant, the H L $\alpha$ line at $1216 \AA$, is completely suppressed.

The $\mathrm{N}_{2} \mathrm{LBH}$ bands are measured in two wavelength segments, as mentioned above: $\mathrm{LBH}$ (short) and $\mathrm{LBH}$ (long). Each of these two wavelength ranges contains a relatively bright $\mathrm{N}$ I line $(1493 \AA$ and $1743 \AA$, respectively). However, the atomic lines are created by the same auroral process (electron impact on $\mathrm{N}_{2}$ ) as the $\mathrm{N}_{2}$ LBH bands and the cross-sections are known (Ajello and Shemansky, 1985), and so the emission in these lines is not treated as a contaminant, but is used in the 


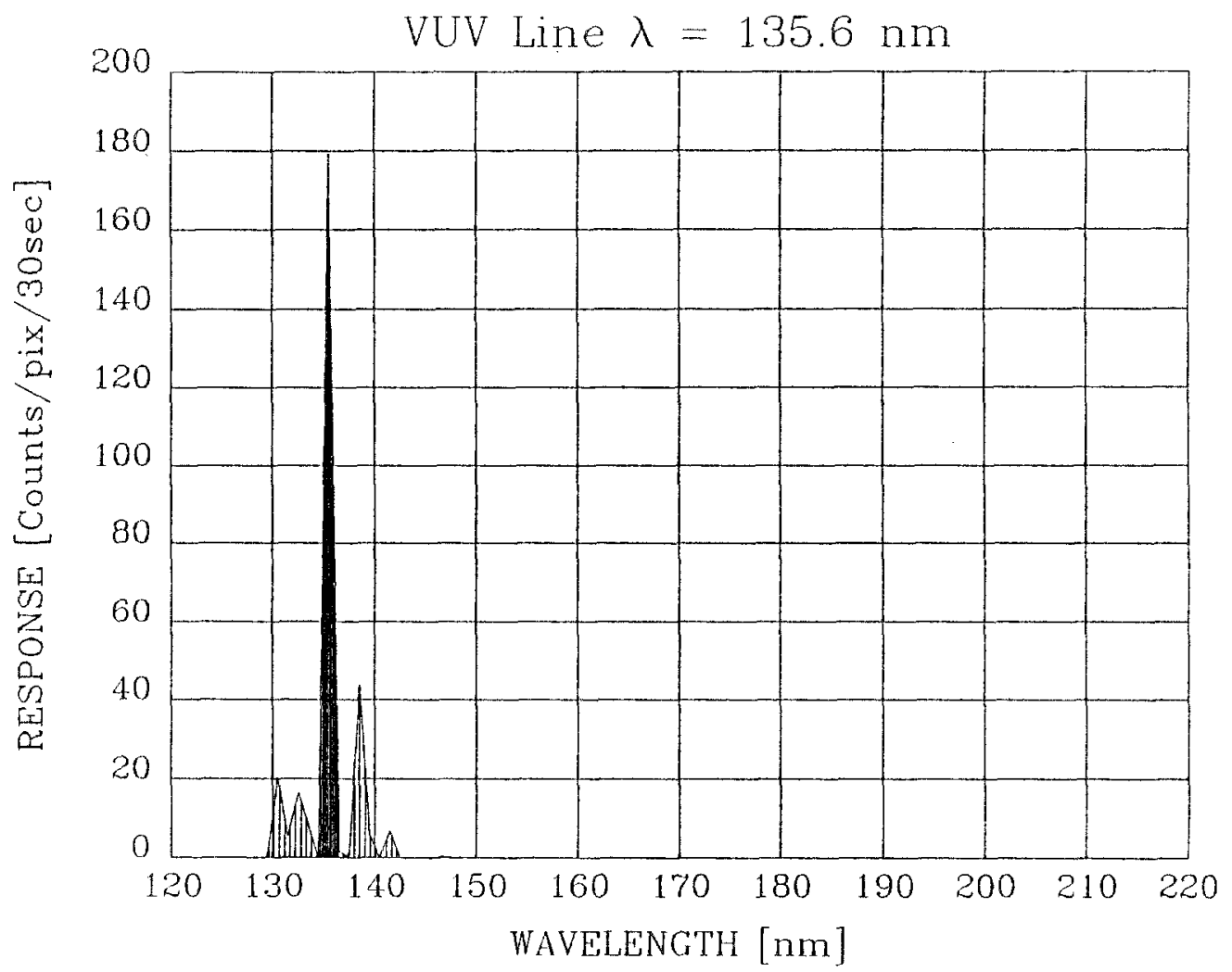

Fig. 2.4.7b. Contribution function for the auroral spectral features passed by the $1356 \AA$ channel.

analysis together with the $\mathrm{LBH}$. The $\mathrm{N}_{2} \mathrm{LBH}$ filters and contribution functions are shown in Figures 2.4.9(a) and 2.4.9(b) and 2.4.10(a) and 2.4.10(b), respectively, for the short and long wavelength segments. Corrections for any neighboring emission leakage is again made, if needed, by Fourier decomposition.

In order to correct for scattered sunlight when and if necessary, the filter and contribution function shown in Figures 2.4.11(a) and 2.4.11(b) is used.

The filter characteristics shown in part (a) of Figures 2.4.7 to 2.4.11 do not include the response of the four $\mathrm{Al} / \mathrm{MgF}_{2}$ coated mirrors while the instrument response shown in Figures 2.4.7(b) to 2.4.11(b) include all filtering and imaging elements of the instrument. The total filter characteristics on their own (without $\mathrm{Al} / \mathrm{MgF}_{2}$ coated mirrors) are shown in Figure 2.4.12. These UVI filters, which were built at the University of Alabama in Huntsville, should be compared with those otherwise available and shown in Figure 2.4.3.

During the course of the nominal 2-year mission of POLAR, instruments on the despun platform will be exposed to a substantial radiation dosage of $275 \mathrm{krads}$. While components, such as filters and electronic parts, that are within the body of the instrument will see radiation exposures of more than an order of magnitude less, 
1304 FILTER

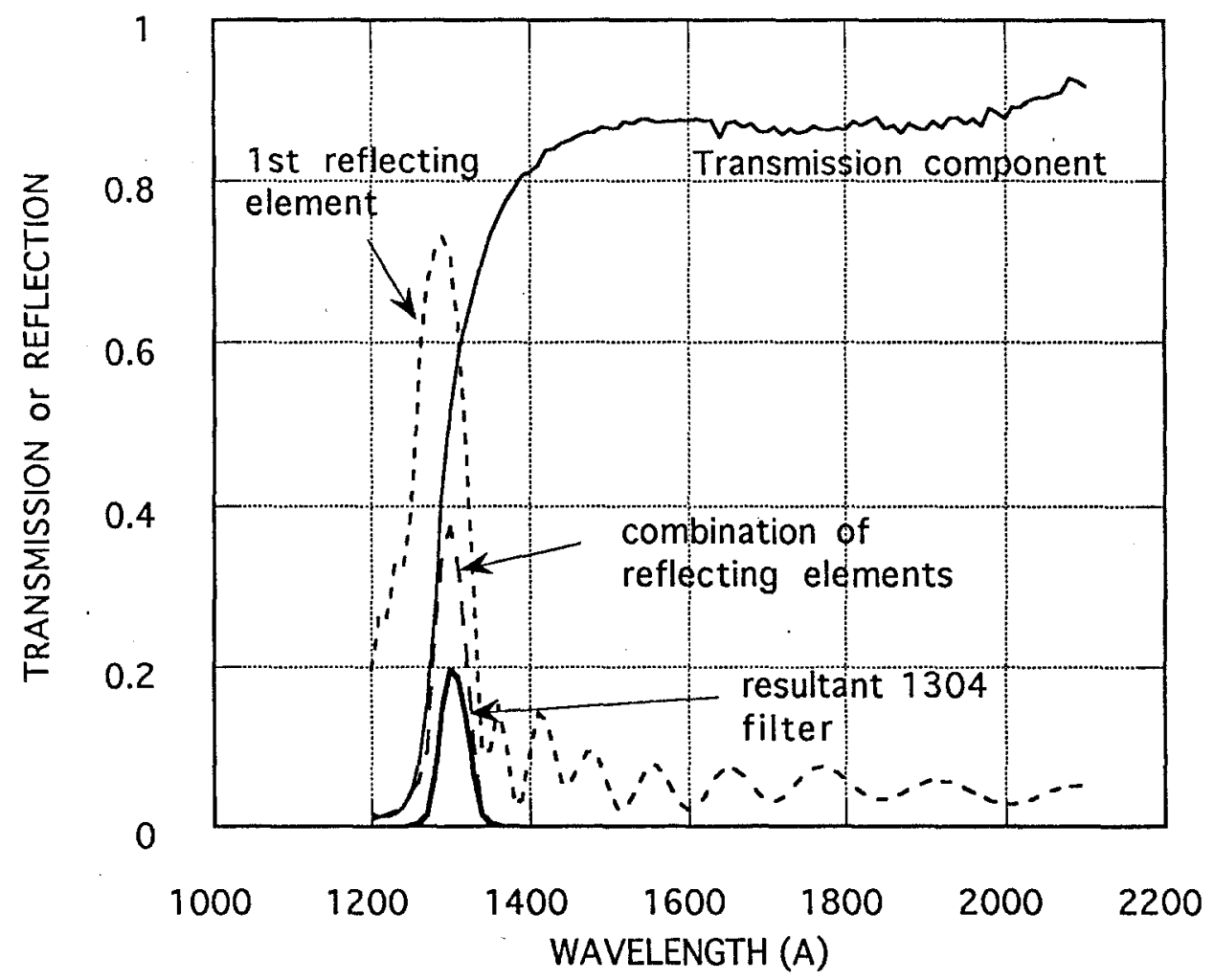

Fig. 2.4.8a. Response of the reflective (solid) and transmissive (dashed) components of the filter elements used for measuring the OI line at $1304 \AA$, and of their combination (cross hatched).

we have conducted extensive tests on the radiation hardness of the filters. These tests are described by Keffer et al. (1993). Two test samples were made of each filter substrate and each thin film type. These samples were carefully characterized as a function of wavelength. One set was then subjected to irradiation of 250 krads from a ${ }^{60} \mathrm{Co}$ gamma radiation source. No measurable changes occurred below $1800 \AA$, although a 2-5\% absorption near $2600 \AA$ was indicated in some of the samples with $\mathrm{MgF}_{2}$ substrates. We therefore do not anticipate any detectable radiation induced changes in the filters during the mission.

As will be seen from the following sections, the instrument contains a fully redundant detector system. This is used by moving a folding mirror into the optical path. When this second channel is used, the response is modified by the reflectivity of an additional $\mathrm{Al} / \mathrm{MgF}_{2}$ surface. 


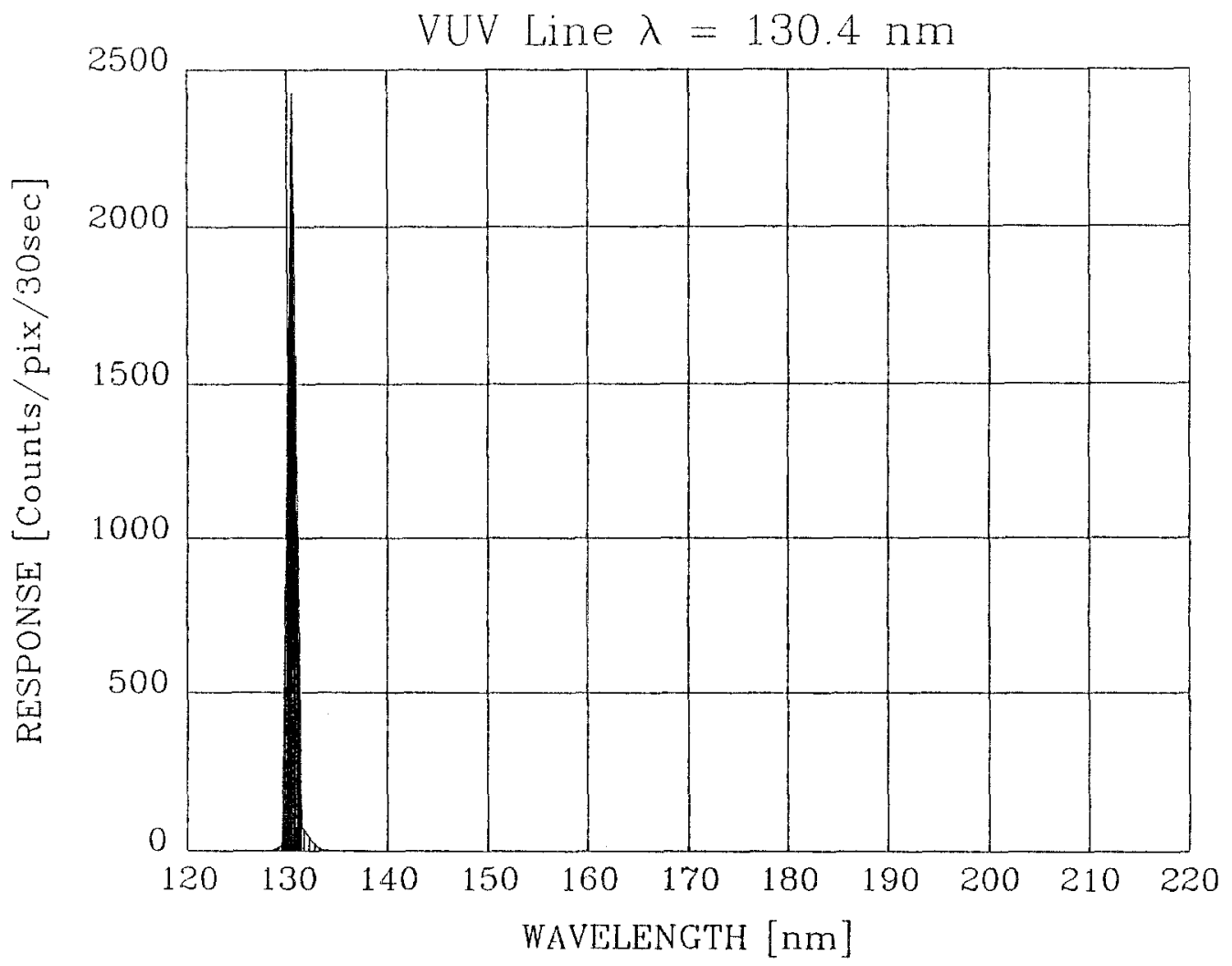

Fig. 2.4.8b. Contribution function for the auroral spectral features passed by the $1304 \AA$ channel.

\subsection{SENSITIVITY}

The sensitivity of the instrument to a line source of $1 R$ intensity (where $1 R$ corresponds to a surface brightness of $10^{6}$ photons $\mathrm{cm}^{2} \mathrm{~s}^{-1}$ radiated into $4 \pi$ steradians) is given by

$$
S=\frac{10^{6}}{4 \pi} \cdot A \Omega \cdot \epsilon \cdot q_{e},
$$

where $A$ is the entrance aperture, $\Omega$ is the solid angle subtended by the instrument field of view, $\epsilon$ is the combination of filter responses and mirror reflectivities, and $q_{e}$ is the quantum efficiency of the detector.

For the configuration shown in Figure 2.3.1, the entrance aperture, $A$, is $11.75 \mathrm{~cm}^{2}$. With an $8^{\circ}$ full angle field of view, the solid angle, $\Omega$, is $0.0153 \mathrm{sr}$. Assuming that the typical detector quantum efficiency is 0.15 over the range $1300 \AA$ to $1900 \AA$, and that the net filter response (including mirrors) is 0.05 at the wavelengths of interest:

$$
S=107 \text { counts } \mathrm{R}^{-1} \mathrm{~s}^{-1} \text { or } S=3966 \text { counts } \mathrm{R}^{-1} \text { over the } 37 \text {-s integration }
$$
period. 
LBHS FILTER

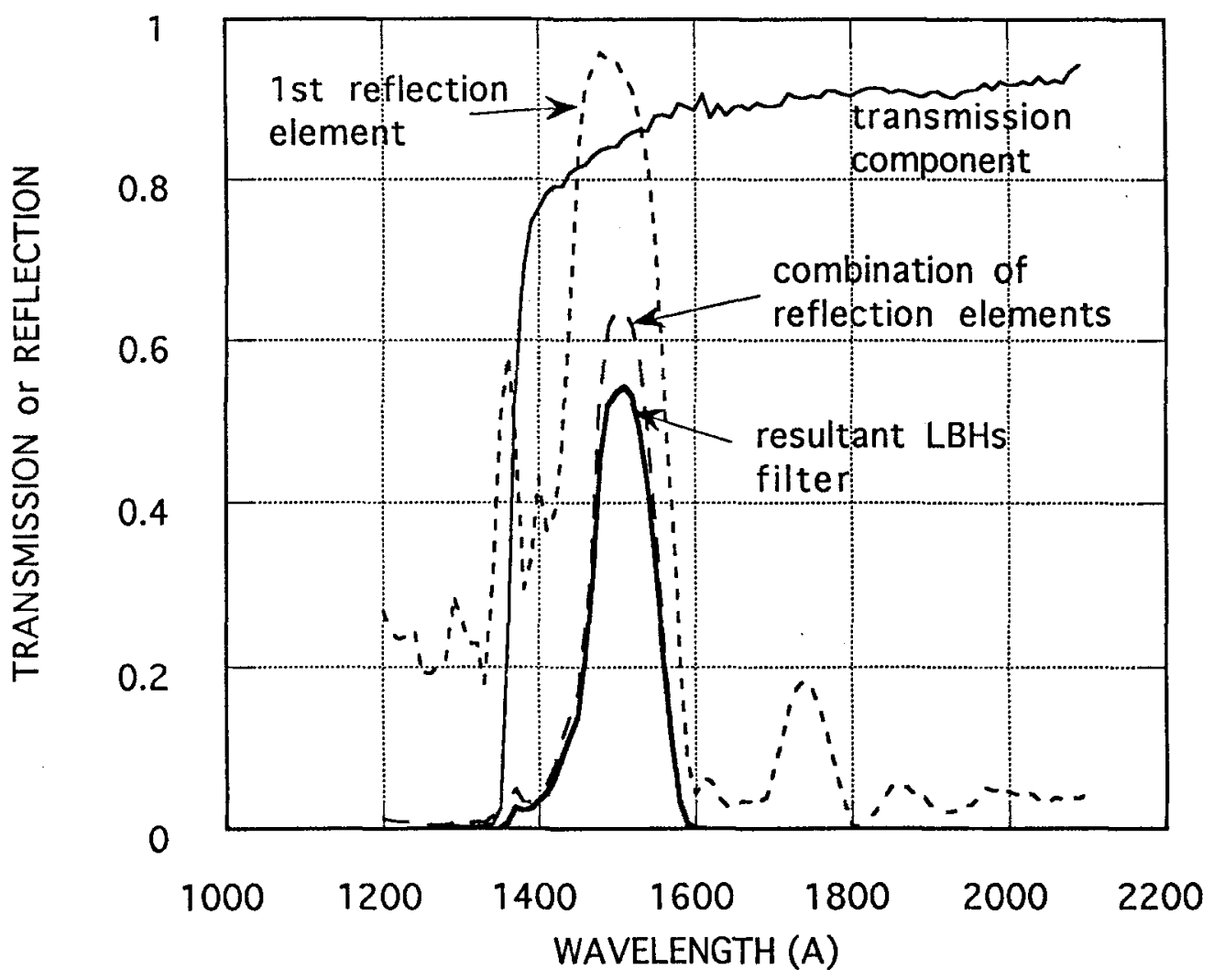

Fig. 2.4.9a. Response of the reflective (solid) and transmissive (dashed) components of the filter elements used for measuring the shorter wavelength LBH bands at 1400-1600 $\AA$, and of their combination (cross hatched).

Discarding the corners of the CCD that see no light, the camera images onto a circular area of 36728 spatial elements, so the sensitivity per spatial element, $S_{\mathrm{E}}$, is

$$
S_{\mathrm{E}}=0.1 \text { counts } \mathrm{R}^{-1} \text { integration period }{ }^{-1} \text { pixel }^{-1} .
$$

Thus, the noise equivalent signal in one integration period is $\sim 10 R$, or $250 R$ will be measured with a signal to noise ratio of 5 . The detector system noise is discussed in Section 2.7 below and does not significantly alter these estimates. Weaker signals or signals at wavelengths where the sensitivity is lower will be measured by coherent summing over longer time periods. Actual sensitivities for each filter as a function of wavelength are shown in Figure 2.5.1. 
TABLE 2.6.1

Typical levels for various FUV components

\begin{tabular}{llcl}
\hline Wavelength & Nightglow $(R)$ & Dayglow or aurora $(R)^{\mathrm{a}}$ & Scattered sunlight $(R / A)$ \\
\hline 1304 & $50-100^{\mathrm{b}}$ & $10000-20000$ & \\
1356 & $50-100^{\mathrm{b}}$ & $1000-2000$ & \\
$1400-1700$ & none & $600-1200$ & \\
$1700-2000$ & none & $300-600$ & $50-500^{\mathrm{c}}$ \\
\hline
\end{tabular}

${ }^{\mathrm{a}}$ From Figure 2.4.1(a).

${ }^{b}$ Tropical arcs only.

${ }^{c}$ From the data shown in Figure 2.4.2.

TABLE 2.6.2

Direct solar fluxes

\begin{tabular}{lll}
\hline $\begin{array}{l}\text { Wavelength } \\
\AA\end{array}$ & $\begin{array}{l}F \\
\text { kR/A }\end{array}$ & Reference \\
\hline 1300 & 0.06 & 1 \\
1350 & $0.1-0.3$ & 2 \\
1400 & $0.1-0.3$ & 2 \\
1500 & $0.3-0.7$ & 2 \\
1600 & $0.8-1.5$ & 2 \\
1700 & $4-6$ & 2 \\
1800 & 10 & 3 \\
1900 & 20 & 3 \\
2000 & 80 & 1 \\
2100 & 300 & 1 \\
\hline
\end{tabular}

References:

1 - Lean (private communication, 1991).

2 - Torr et al. (1980).

3 - Heroux and Swirbalus (1976).

TABLE 2.6.3

Out of field stray light analysis

\begin{tabular}{lllll}
\hline Wavelength, $\AA$ & $\begin{array}{l}\text { Mean background } \\
\end{array}$ & \multicolumn{4}{l}{ Straylight throughput-In band $9 R_{\mathrm{E}}\left(\mathrm{W} \mathrm{cm}^{-2}\right)$} \\
\cline { 3 - 5 } & & $0 \mathrm{deg}$ & $90 \mathrm{deg}$ & $135 \mathrm{deg}$ \\
\hline $1191-1241$ & $7.35(-08)$ & 0 & 0 & $2.79(-14)$ \\
$1279-1304$ & $4.57(-08)$ & 0 & 0 & $7.59(-14)$ \\
$1331-1381$ & $4.39(-08)$ & 0 & 0 & $1.28(-13)$ \\
$1400-1700$ & $2.26(-09)$ & 0 & 0 & $8.42(-12)$ \\
$1468-1518$ & $1.33(-10)$ & 0 & 0 & $4.1(-13)$ \\
$1700-2000$ & $9.46(-10)$ & $5.04(-14)$ & $1.00(-14)$ & $2.06(-11)$ \\
$2125-2175$ & $7.39(-09)$ & $2.54(-14)$ & $5.08(-15)$ & $9.69(-12)$ \\
\hline
\end{tabular}




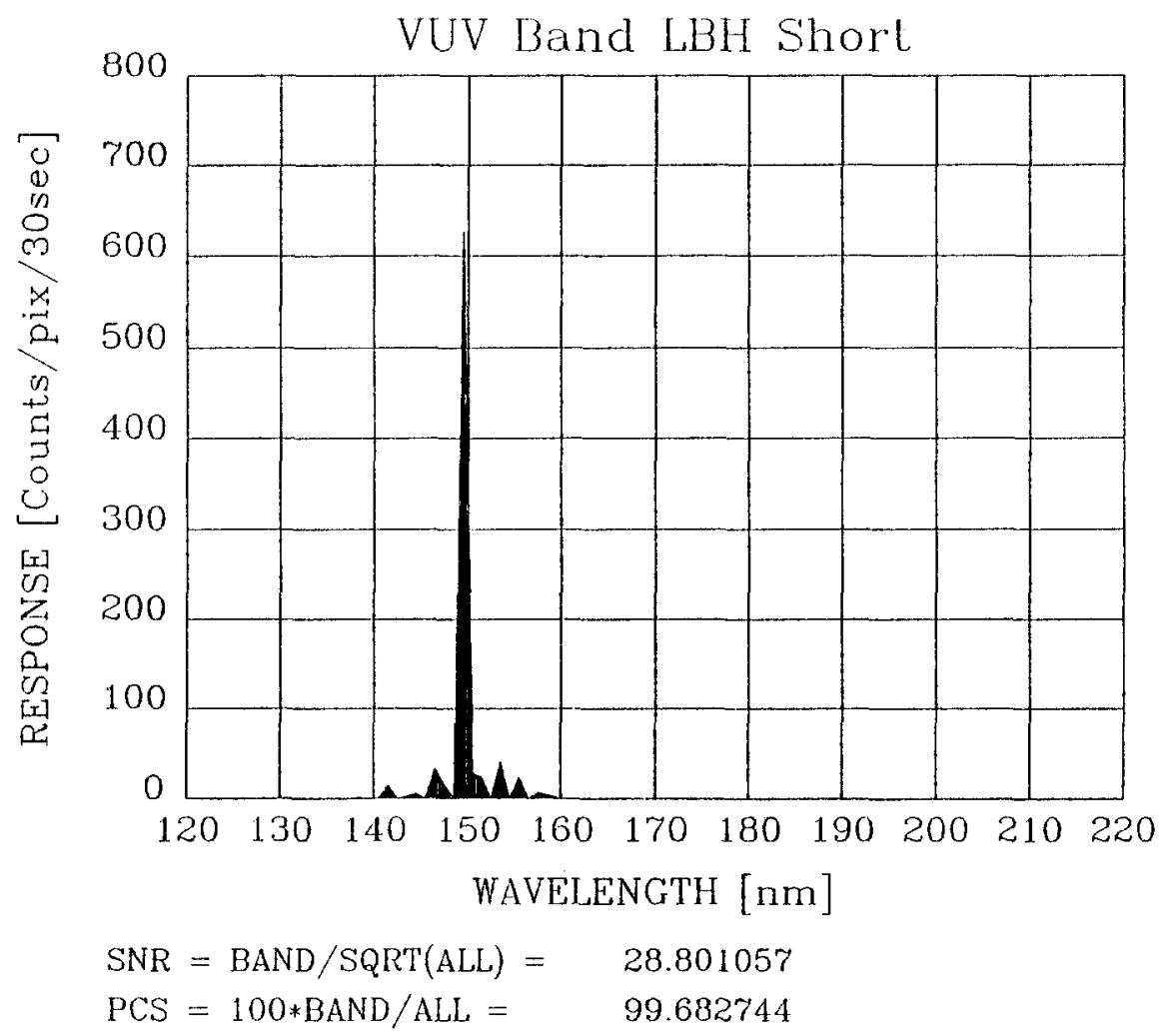

Fig. 2.4.9b. Contribution function for the auroral spectral features passed by the shorter wavelength LBH(short) channel.

In designing the UVI, there are three major sources of stray light with which we have to contend, and each represents a design issue that must be addressed. These three are: (1) out-of-band light; (2) out-of-field light; and, (3) very bright, in-band, in-field scene regions that can scatter onto and swamp weak in-field regions.

To establish the acceptable thresholds of measurement, we have taken the following approach: In a perfect situation of no out-of-band or out-of-field light, the auroral signal must be measured on top of the background of the naturally occurring airglow. Typically, the dayglow and the aurora are comparable, while the aurora is generally much brighter than the nightglow, which in the FUV is essentially non existent (except for the atomic oxygen line at 1304/1356A in the equatorial arcs and the ubiquitous hydrogen geocorona). Working levels for the competing components in the FUV are given in Table 2.6.1.

Typical fluxes of direct sunlight in the FUV are shown in Table 2.6.2.

We have dealt with each of the stray light sources as follows:

The out-of-band light comprises the long wavelength (primarily visible) sunlight scattered off the Earth and lower atmosphere, and FUV auroral/dayglow features 
LBHI FILTER

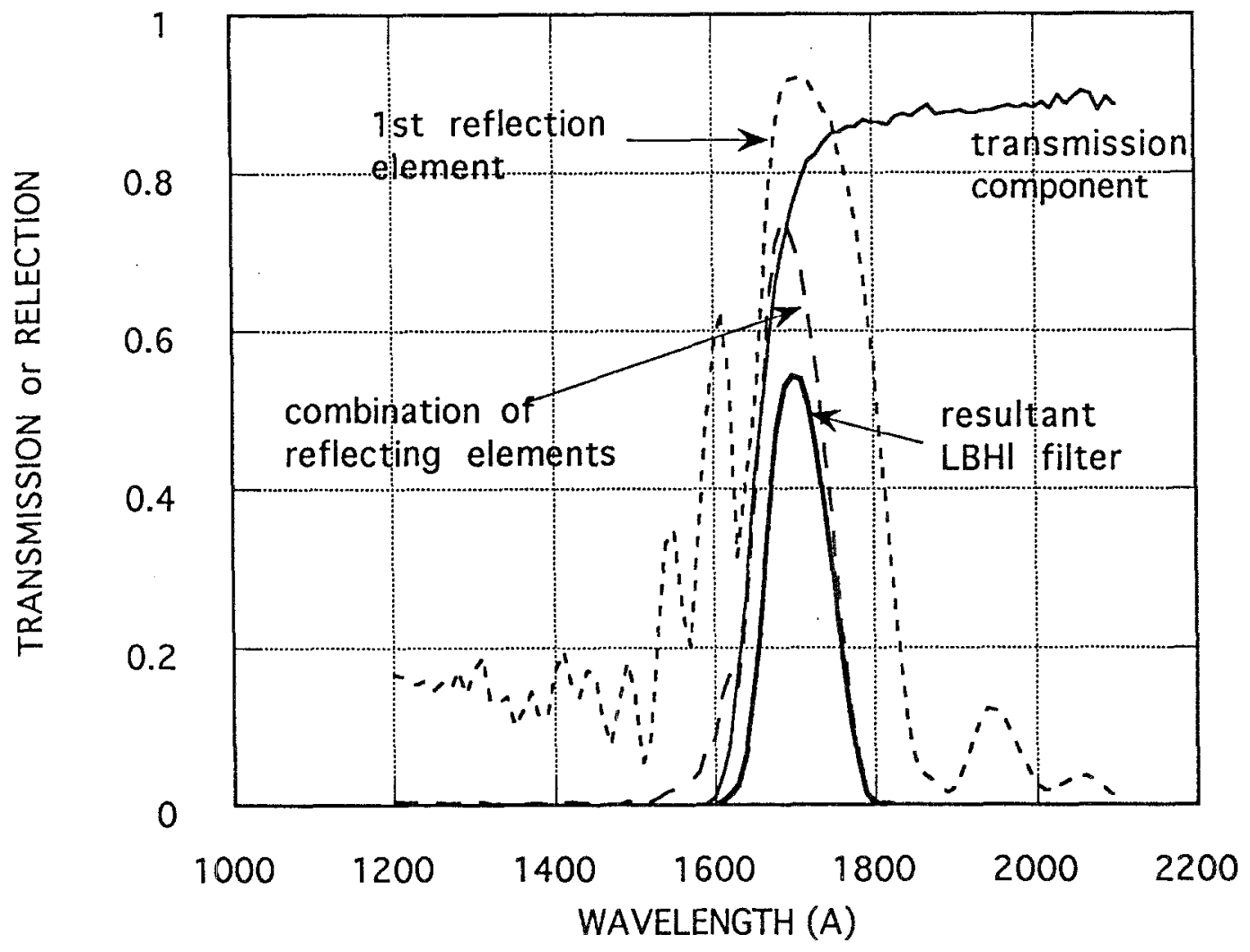

Fig. 2.4.10a. Response of the reflective (solid) and transmissive (dashed) components of the filter elements used for measuring the LBH bands at $1600-1900 \AA$, and of their combination (cross hatched).

that have some contribution in the bandpass of a neighboring filter. These have been adequately dealt with in the filter design, as discussed in Section 2.3 above.

The out-of-field light is rejected by careful baffle design, including both a sun shade and internal baffling. Since the long wavelength light is rejected as discussed above, we will consider only the in-band out-of-field light here.

Again, our goal was to reduce stray light to a level below that of the naturally occurring airglow. For this purpose, a detailed analytical model of the UVI was developed. The inner surface of the baffle is coated with Chemglaze (Z306). Using the natural contributions given in Table 2.6.1, the scattered component was modeled for various angles of illumination and the extremes of the orbit. The results are shown in Table 2.6.3, from which it can be seen that the baffle achieves the required attenuation.

Specular scattering within the camera, such as might occur off the edges of mirrors, etc., has been almost entirely contained by using our capability to merge 


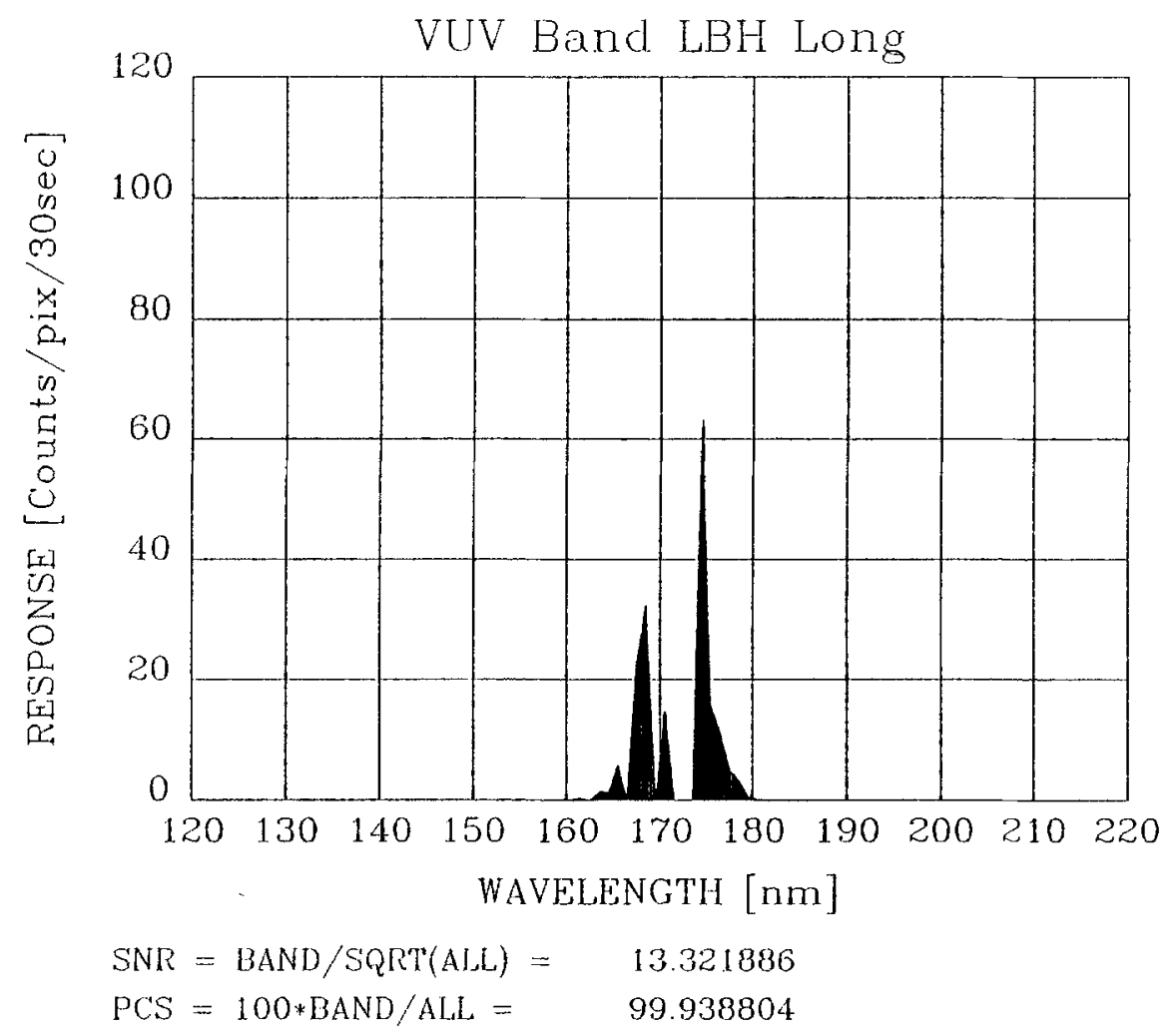

Fig. 2.4.10b. Contribution function for the auroral spectral features passed by the LBH(long) channel.

the ray trace (SYNOPSIS) and the CAD mechanical design products (Figure 2.6.1). This has been used to design carefully sculptured pieces that baffle to the edge of the cone of light between the primary and secondary mirrors, and again between the secondary and tertiary mirrors. An example of one of these enclosures is shown in Figure 2.6.2.

The very bright in-field regions are a concern in that this light can potentially scatter off the optical surfaces and contaminate the weaker regions of the image. The instantaneous dynamic range of the detector is 1000 , and so in order to utilize this range, we need to reduce in-field scatter to less than $10^{-3}$. For this reason, we have placed great emphasis on the microstructure and mid-frequency surface roughness as discussed above. Our approach here has been to fabricate optics with the best surfaces that present technology will allow. For clean optics with the surface roughness discussed above, the scatter requirements should be met.

In addition to the above three stray light issues, there is a fourth aspect which we have also assessed. Ideally, the instrument field of view should never be penetrated by parts of the spacecraft, but unfortunately the satellite configuration is such that this does occur. This arises from the fact that one of the electric field measuring 
SOLAR FILTER

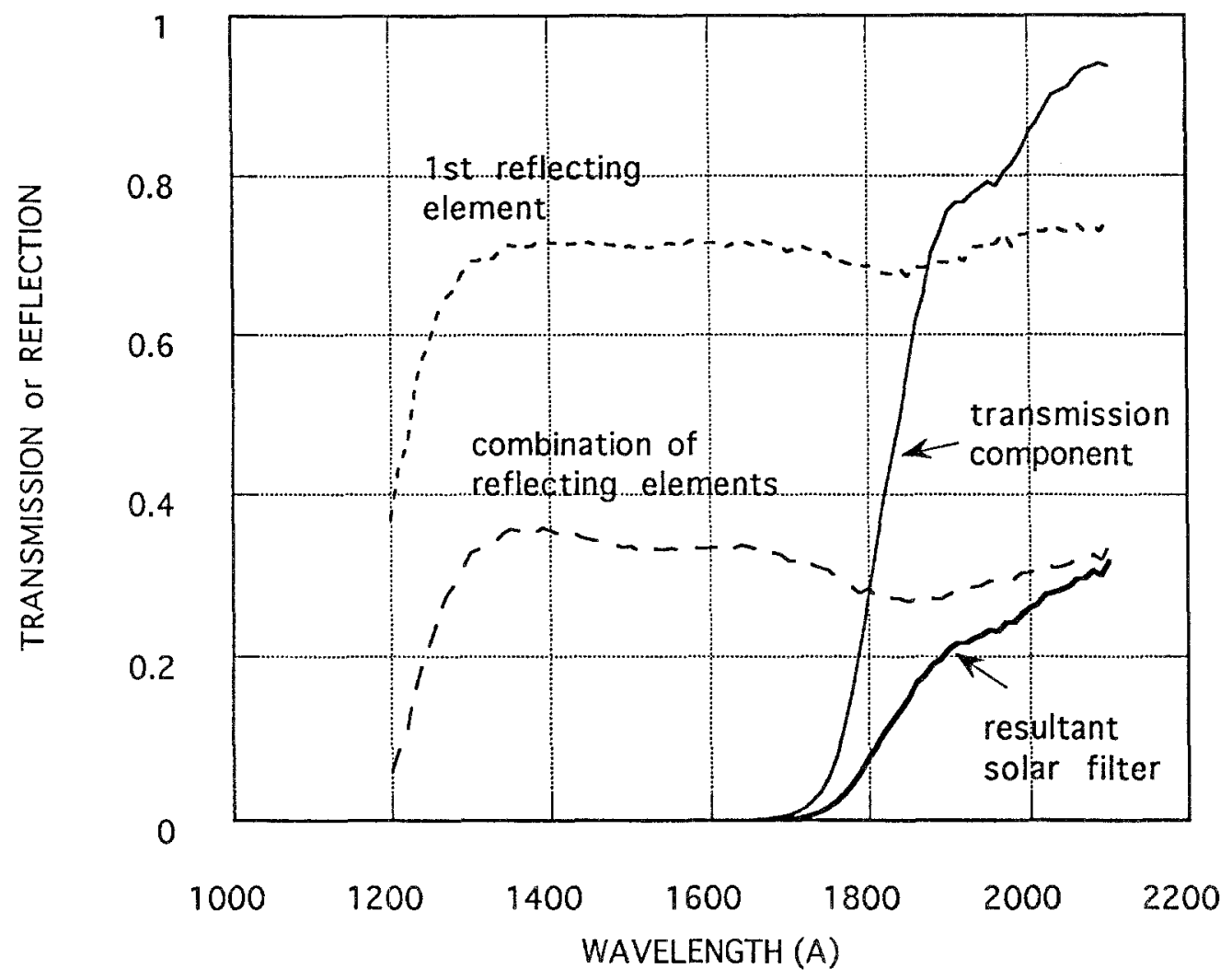

Fig. 2.4.11a. Response of the reflective (solid) and transmissive (dashed) components of the filter elements used for measuring the scattered sunlight spectrum, and of their combination (cross hatched).

instruments on the POLAR spacecraft deploys four antennas, at the ends of which are spheres. Two of the antennas are $80 \mathrm{~m}$ long and two are $50 \mathrm{~m}$ long. The four antennas are $90^{\circ}$ apart. The antennas are $\sim 0.1 \mathrm{~cm}$ in diameter, and the spheres at the ends are $10.16 \mathrm{~cm}$ in diameter. Because of the length of the antennas and the fact that they are attached to the spinning body of the spacecraft, these four objects will pass through the field of view of the imagers on the despun platform every spacecraft spin cycle. The spin rate is $10 \mathrm{rpm}$, and so one of the antennas will pass through the field of view every $1.5 \mathrm{~s}$. This problem is severe enough at visible wavelengths that the visible imager actually shuts down for the brief period of time that each of the antennas crosses its field of view. We have assessed the problem in the FUV as follows.

The angle subtended by the spheres at 50 and $80 \mathrm{~m}$ respectively is $2.0 \times 10^{-3}$ radians and $1.3 \times 10^{-3}$ radians, respectively. A single pixel subtends approximately $0.6 \times 10^{-3}$ radians, and so the spheres will span a swath of 5 and 2 pixels respectively in their paths across the detector. The width of the antenna wires at $30 \mathrm{~m}$ is 


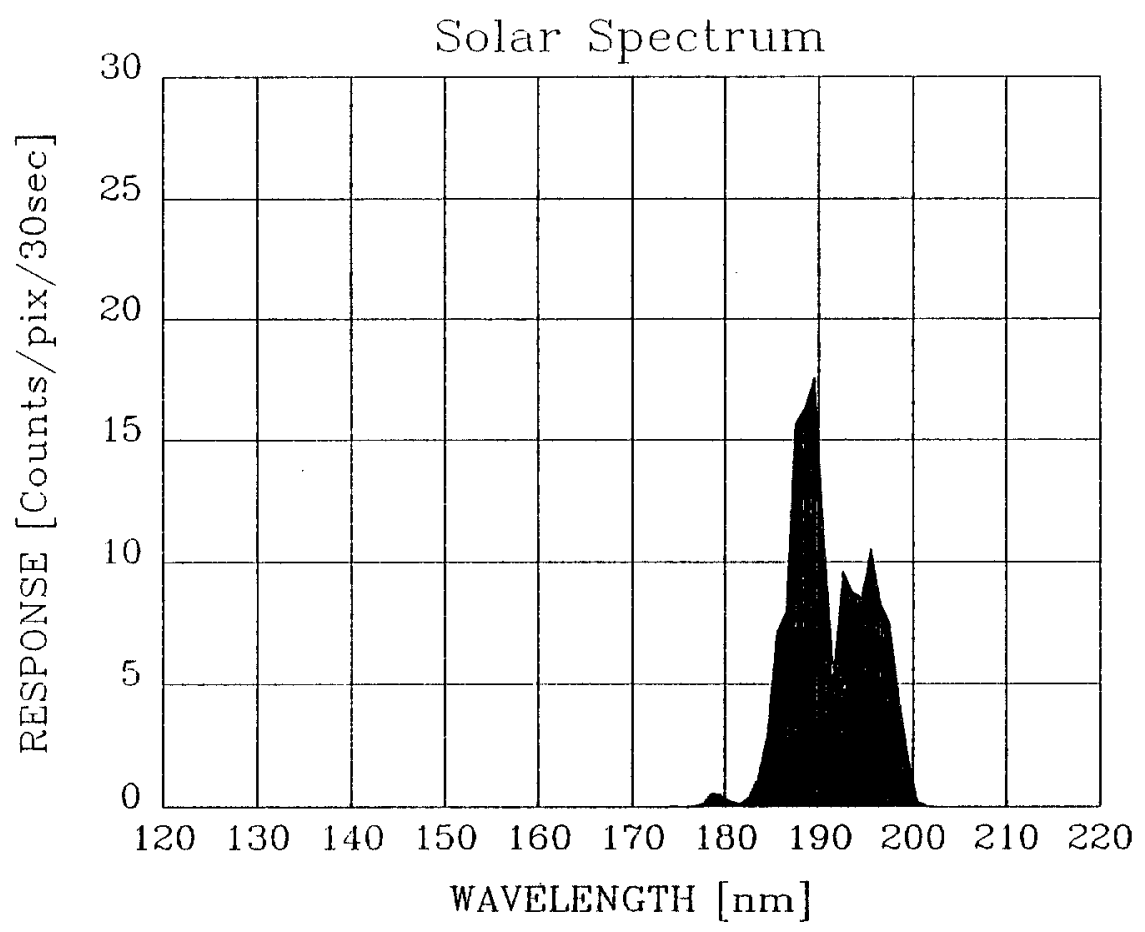

$$
\begin{aligned}
& \mathrm{SNR}=\mathrm{BAND} / \mathrm{SQRT}(\mathrm{ALL})=12.148685 \\
& \mathrm{PCS}=100 * \mathrm{BAND} / \mathrm{ALL}=\quad 99.967142
\end{aligned}
$$

Fig. 2.4.11b. Contribution function for the scattered sunlight passed by the solar spectrum channel.

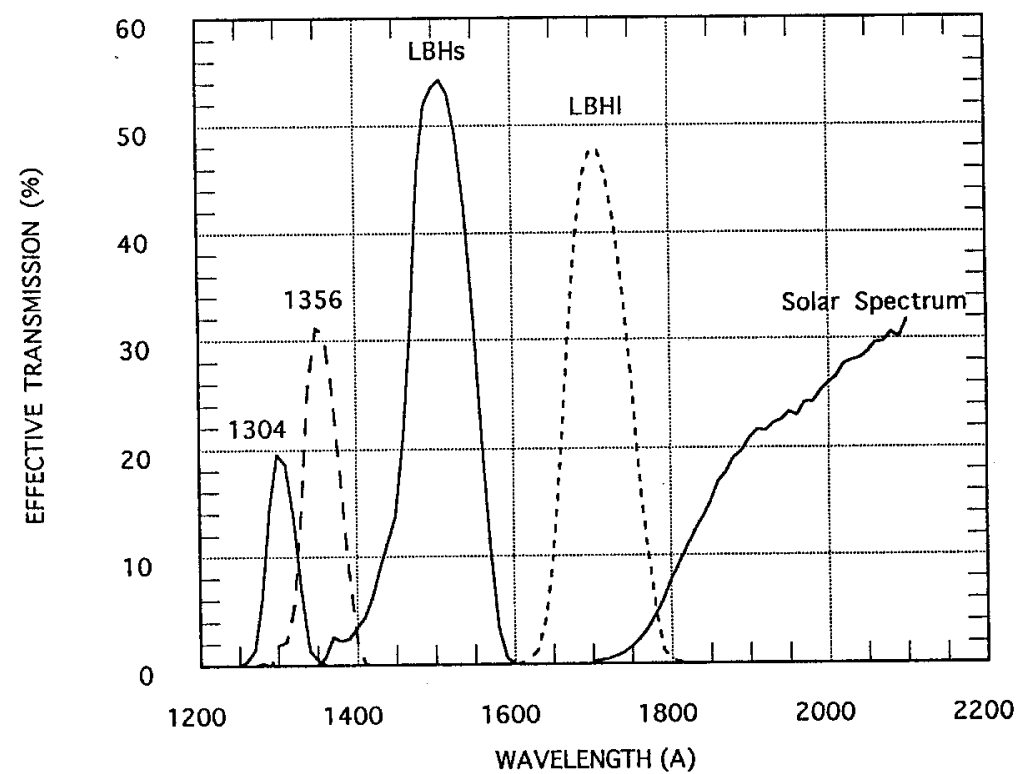

Fig. 2.4.12. Effective transmission of the filters (without the contribution of the three aspheric reflective mirrors, and the folding mirror). 


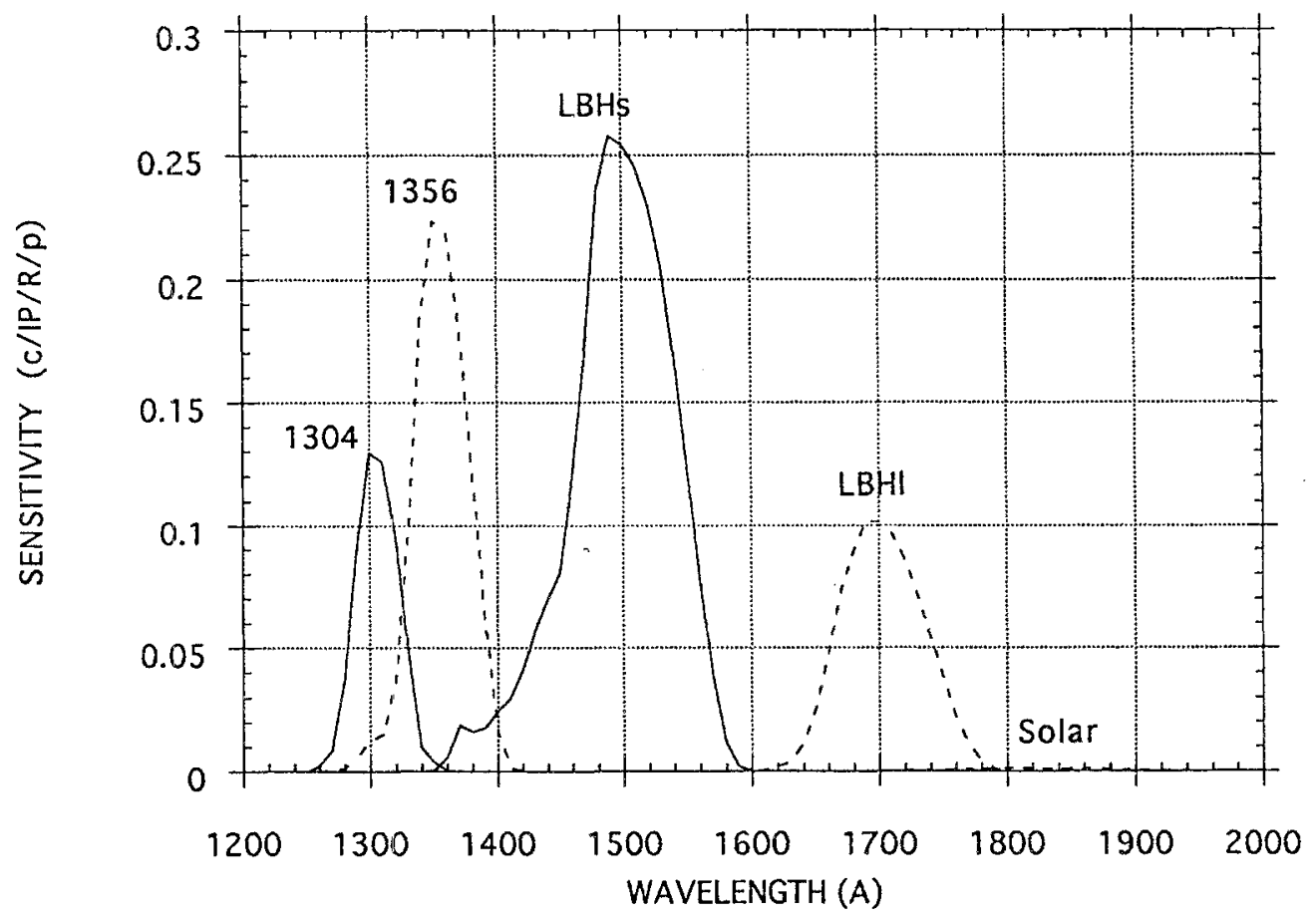

Fig. 2.5.1. Sensitivity of the instrument for each filter as a function of wavelength. The units are counts per integration frame per Rayleigh per pixel.

TABLE 2.6.4

Equivalent brightness of E-field spheres (worst case)

\begin{tabular}{lccl}
\hline Filter A & Bandwidth $\AA$ & F kR/A & Equivalent brightness $R$ \\
\hline 1304 & 50 & 0.06 & 0.002 \\
1356 & 70 & 0.3 & 0.017 \\
LBH-short & 175 & 0.5 & 0.07 \\
LBH-long & 80 & 5 & 0.32 \\
Solspec. & 90 & 20 & 1.44 \\
\hline
\end{tabular}

approximately 0.05 pixel. The antennas are attached to the spacecraft approximately $1 \mathrm{~m}$ below the despun platform. As a result, the spheres on the 50 and $80 \mathrm{~m}$ antennas will cross the detector with a separation of approximately 12 pixels. The issue is whether or not these will leave severe spurious streaks across the images.

We have obtained samples of the sphere surface material and have measured the FUV reflectivity. From these measurements we have set an upper limit of 0.005 on the reflectivity, as it is too small for us to measure accurately.

Using these values, and assuming that the spheres, as seen by the UVI, are fully illuminated by the Sun and, further, assuming the worst case situation in which all 


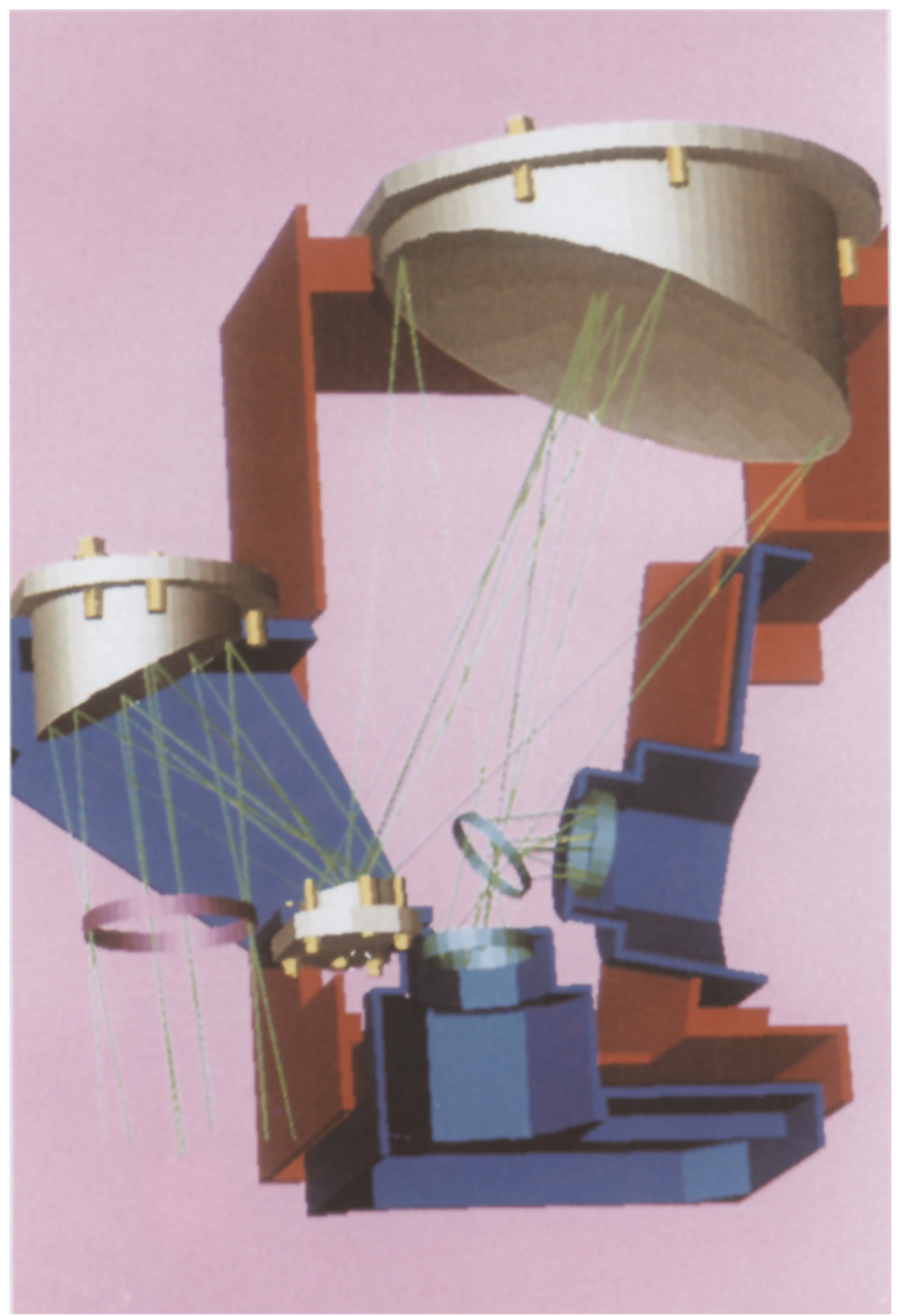

Fig. 2.6.1. CAD design of optical bench, with ray trace superimposed.

the light is somehow specularly reflected back into the instrument field of view, we obtain the equivalent brightnesses summarized in Table 2.6.4. The solar flux values used are those for solar maximum and so also represent an upper limit.

We conclude that these antennas, if constructed to the planned parameters (i.e., those used in the above analysis), should not significantly affect the images. 


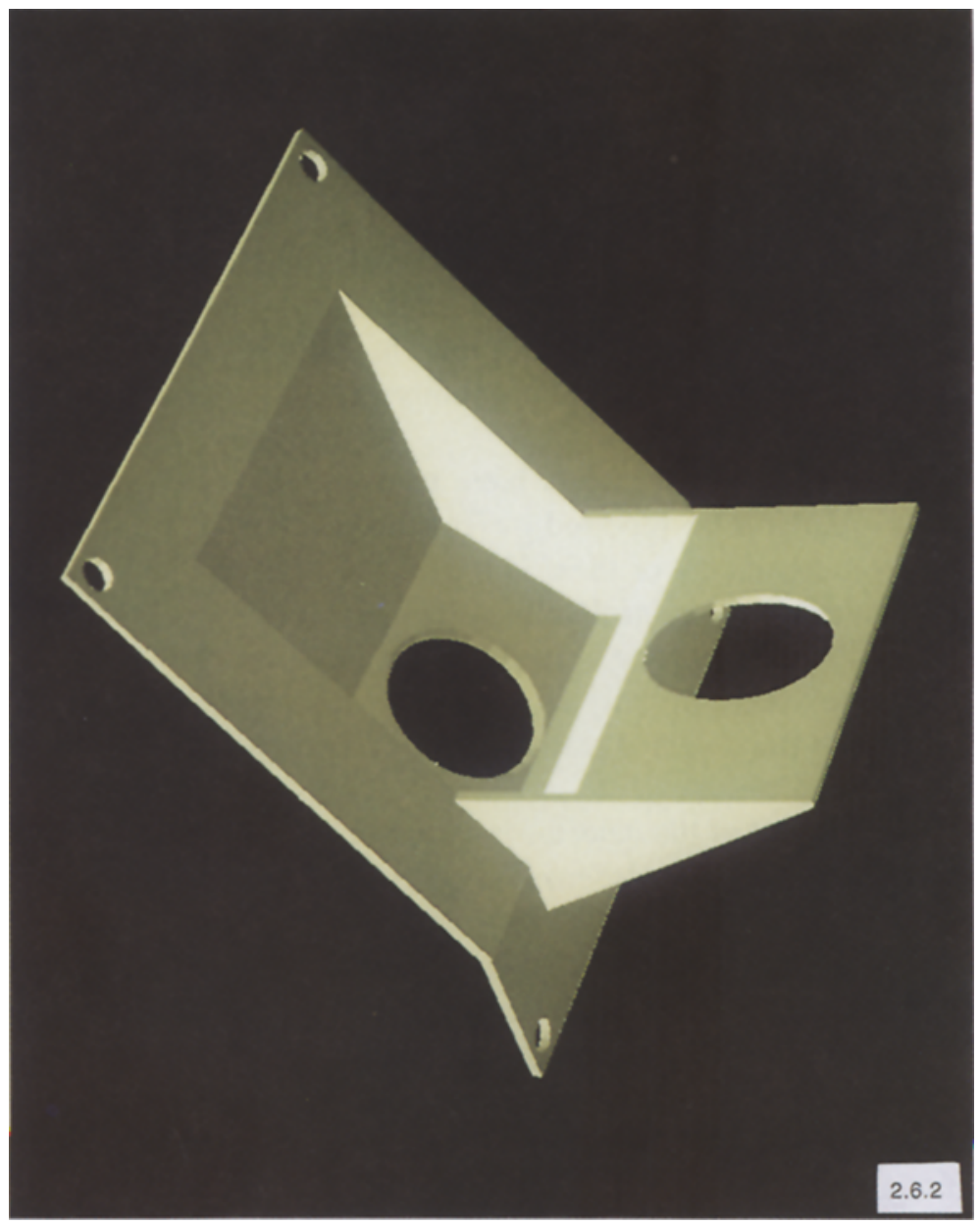

Fig. 2.6.2. One of the baffles used in the interior of the camera to completely isolate the various mirror sections.

\subsection{DETECTOR SYSTEM}

The detector system used here is an intensified-CCD system. The detector represents an evolution of the intensified-CCD detectors that we have used in other instruments (Torr and Devlin, 1982; Torr et al., 1986). It is described in more detail by Torr et al. (1993) and comprises several key elements. The first of these is a proximity focused image intensifier with chevron microchannel plates. The intensifier, manufactured by SAIC, has an opaque CsI photocathode deposited on the front surface of the first microchannel plate (MCP). The $\mathrm{MgF}_{2}$ faceplate is only $3 \mathrm{~mm}$ thick in order to maximize the throughput. The combination of thin, planar faceplate and opaque photocathode allows high effective quantum efficiencies to be achieved and high spatial resolution within the intensifier. The inside surface 
of the $\mathrm{MgF}_{2}$ window has a thin film of nichrome deposited on it, so that it can be properly biased. The MCP is a ' $\mathrm{V}$ ' plate in which two plates, each cut on the bias, are mounted in series. In combination these plates can achieve an electron gain with an upper limit in the range of $10^{4}$ to $5 \times 10^{4}$. By reducing the voltage across the $\mathrm{MCP}$, the electron gain can be reduced. Luminous gains down to 1 can be reached. The electrons are further accelerated across to a phosphor (P31) deposited on the fiber optic stub which is the tube output. A further amplification of $\sim 60$ is achieved at the phosphor for the voltage difference used in our system. The elements of the intensifier are mounted in very close proximity to each other and thus the image quality is maintained.

Several significant improvements have been incorporated into the detector design since the early work reported by Torr and Devlin (1982) and Torr et al. (1986). The most significant of these is the design of a very stable high voltage power supply for the MCP control voltage. This is stable to $25 \mathrm{ppm}$ per ${ }^{\circ} \mathrm{C}$ and is essential to reliable interpretation of the intensity measurements. In addition, CCD electronic noise has been virtually eliminated by the incorporation of correlated double sampling in the detector readout electronics. Upgrades are described by Torr et al. (1995).

The readout element of the detector is a charge coupled device (CCD), a bulk silicon array of $488 \times 550$ photosensitive pixels, in a frame transfer format. The device used is the Thomson-CSF TH 7866 chip which is a front-side illuminated, buried channel design. In the frame transfer format, the CCD has 2 side-by-side arrays, one of which is photosensitive, and one is not. The contents of the active area ( $244 \times 550$ pixels), after light exposure, are clocked into the storage area (in 39 milliseconds) and then integration continues on the active pixels while the storage area is read out, digitized to 12 bits, placed in memory and formatted for telemetry. In the formatting process, the odd and even pixels are added, and the unilluminated corners of the CCD are discarded. No other data compression is done. Typical on-chip integration periods are 4 spacecraft major frames, or $36.8 \mathrm{~s}$.

Because the output fiber optic stub from the intensifier has a numerical aperture of 1 , it is necessary to couple this output directly to the surface of the CCD, or at least to within a spacing of better than $5 \mu \mathrm{m}$. A transfer fiber optic stub is used to achieve this. This transfer fiber optic has a minification chosen to match the dimensions of the intensifier to those of the CCD, as is discussed below. Radiation hard materials are used in the fabrication of the fiber optic taper.

The CCD must be cooled to below $-55^{\circ} \mathrm{C}$ to reduce thermal noise to a negligible level over the long ( $\sim 37 \mathrm{~s})$ on-chip integration periods. This is done using a passive radiative cooler in thermal contact with the back of the CCD package. The passive radiator is located on the top side of the instrument, which is never directly illuminated by sunlight, and will achieve a temperature of $\sim-80^{\circ} \mathrm{C}$ over the course of normal operations.

The active area of the image intensifier is circular with a diameter of $18 \mathrm{~mm}$. The active area of the CCD is $6.6 \times 8.8 \mathrm{~mm}$. The $6.6 \mathrm{~mm}$ dimension comprises 


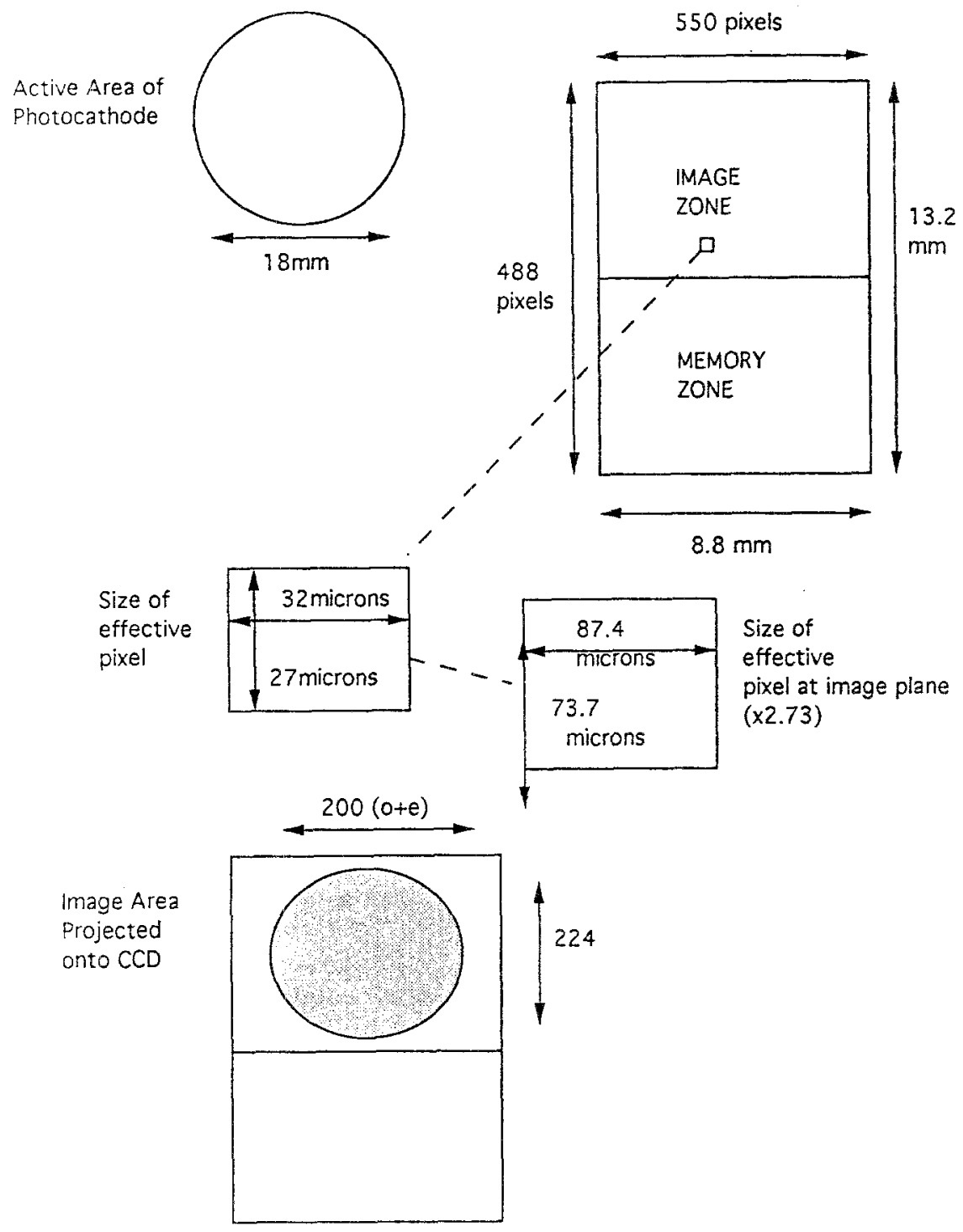

Fig. 2.7.1. Mapping of the image intensifier onto the $\mathrm{CCD}$, showing also the mapping of a single pixel element.

244 pixels with each pixel $27 \mu \mathrm{m}$ in width. The $8.8 \mathrm{~mm}$ dimension comprises 550 (odd-even format) pixels with each pixel $16 \mu \mathrm{m}$ in this dimension. In the UVI application 2 pixels are added in this dimension to yield $244 \times 275$ effective pixels, each $27 \times 32 \mu \mathrm{m}$. To maximize the overlap of CCD and intensifier active areas (one being rectangular and the other round), a fiber optic stub with a magnification ratio of 2.73 is used. Therefore a single CCD pixel is magnified from $27 \mu \mathrm{m} \times 32 \mu \mathrm{m}$ 


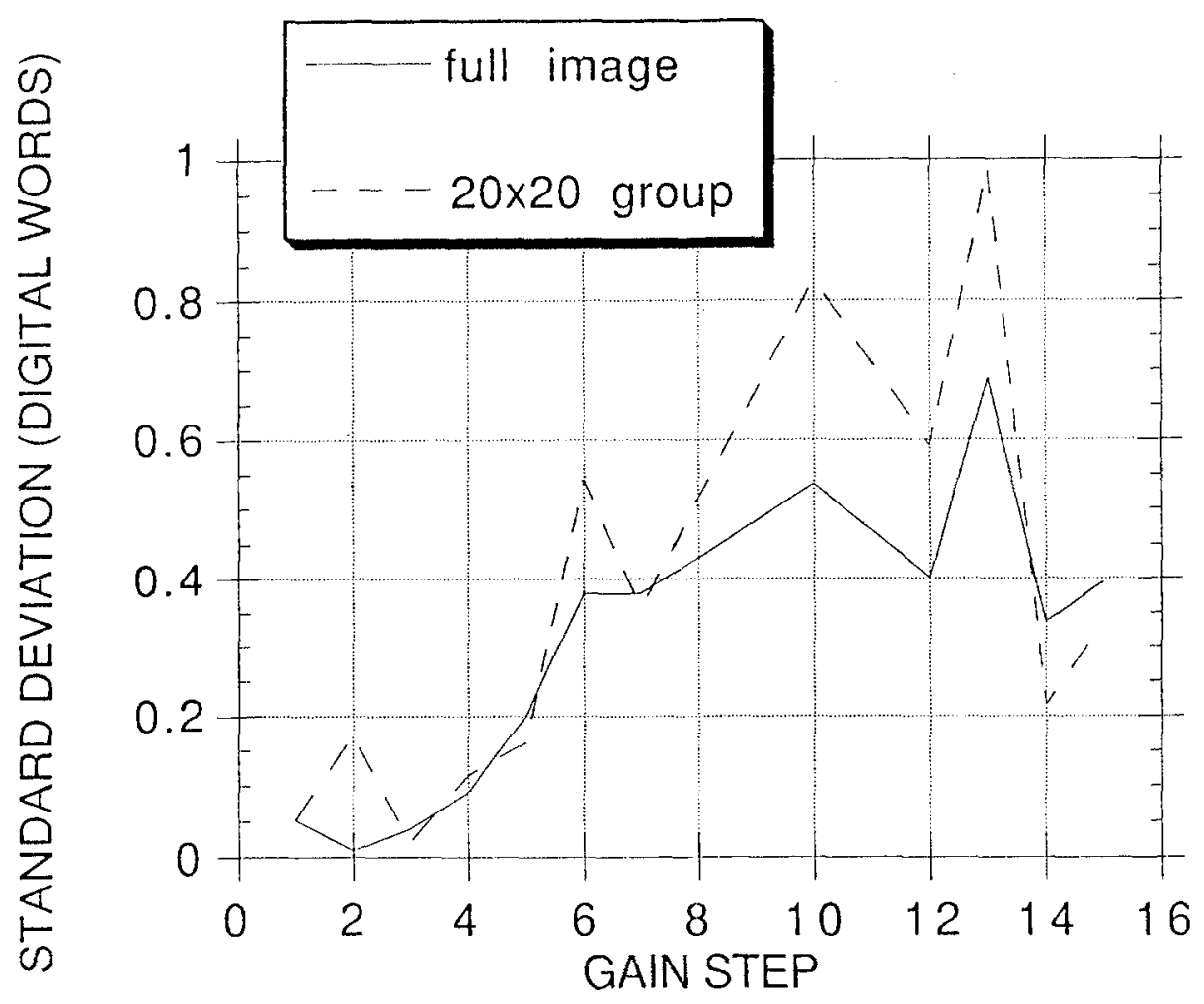

Fig. 2.7.2. The standard deviation (in DW) of a small group of pixels and of the entire device as a function of MCP gain setting.

at the CCD to $74 \mu \mathrm{m} \times 87 \mu \mathrm{m}$ at the focal plane. The geometry is illustrated in Figure 2.7.1. The unilluminated edges of the CCD are discarded, resulting in an oval frame with 200 by 228 pixels diameters.

Relatively little information is available on the long term performance of intensified-CCD systems in a radiation environment. The detector system is well shielded from the softer components of the anticipated radiation by additional housing materials. However, no amount of shielding can protect the device from the very penetrating radiation events such as solar flares, and we expect that the performance will deteriorate as a function of time. There is some evidence that CCDs are less affected when they are powered off. For this and other reasons, the camera has two fully redundant detector systems in the event that one should degrade or fail. The secondary system will only be turned on if our flight calibration procedures (VUV stars) indicate significant changes in the primary system.

Various sources contribute to the total noise of the detector system. Some of these are not readily quantified as separate components, but we will discuss the likely values of each. We will assume that the intensifier contribution to the detector noise is negligible, and indeed this is reasonable, as the image tubes selected for the 
flight instrument were found to be very quiet. From our past experience, the noise incurred in the electronics in reading out the detector every $37 \mathrm{~s}$ is on the order of 30 electrons. Much lower values can be reached with considerable effort, but such designs were not needed here. For the Thomson CCDs used in this instrument, a single pixel will typically contain $5 \times 10^{5}$ electrons when full. When digitized to 12 bits, a digital word (DW) corresponds to 120 electrons at the CCD. Thus the system electronics contributes less than 1DW to the total noise. At $-55{ }^{\circ} \mathrm{C}$, the thermally generated noise in one pixel in $37 \mathrm{~s}$ would be expected to be 1110 electrons, or $9 \mathrm{DW}$. However, the noise on this component is 33 electrons or less than $1 \mathrm{DW}$. We would therefore expect the instrumental noise to be on the order of $1 \mathrm{DW}$ or less for nominal operations. At an intensifier MCP gain of 100, this corresponds to a sky signal of $5 R$. This is less than (but comparable to) the NES for the sky limited statistics discussed in Section 2.5. These estimates are borne out by the actual performance of the instrument. In Figure 2.7.2 we show the standard deviation (in DW) of a small group of pixels and of the entire device as a function of MCP gain setting.

Image intensifiers of the type used here have phosphors on the output stage. A drawback of such phosphors is the fact that they have relatively slow decay times (Torr, 1985). The decay function has 2 components: a prompt or fluorescent emission, and a delayed or phosphorescent emission. For many applications it is only the first of these that is a concern, but for applications such as this in which the dynamic range is large and includes very low light levels, the delayed emission acts as a memory of the previous image(s). In the detector system used here a P31 phosphor is used instead of the more typical P20. The P31 has a faster prompt emission than the P20. To deal with the delayed component, a 9.2-s wait time is built into sequences between changing filters. At the end of this wait time, the detector is cleared. Despite these precautions, one will have to be aware of the potential for phosphor memory effects, which can be verified by checking the previous images.

\subsection{MECHANICAL/THERMAL DESIGN}

A primary requirement of the mechanical system is that it maintain the optical components in precise alignment over the anticipated thermal operating range of the instrument. It must provide the interface to the despun platform, and, since essentially no exchange of heat with the despun platform is allowed, it must provide the means to passively radiate heat generated to space. It must also provide an isolated surface sufficiently cold to allow conductive cooling of the CCDs. The housing that encloses the spectrometer must be completely light tight, other than the entrance aperture, and not permit any stray light paths other than through the optical path and filter system. It must provide the electromechanical devices that move filters and mirrors on command. Because the instrument is mounted on the 


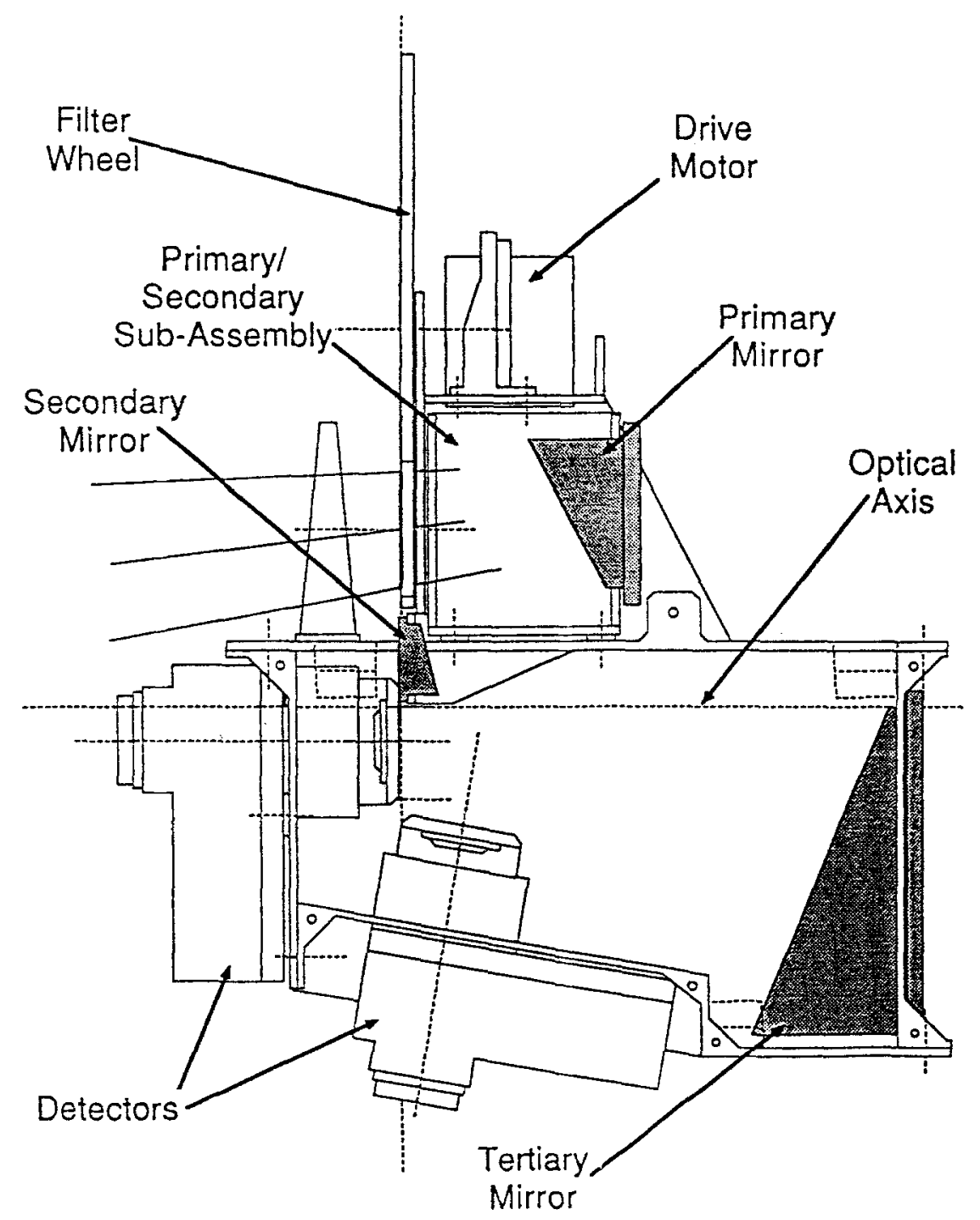

Fig. 2.8.1.1. Schematic of instrument. The $45^{\circ}$ reflective filter elements are not shown.

despun platform, it must meet stringent weight and power requirements: it was designed to fit within an envelope of $21 \mathrm{~kg}$ and $21 \mathrm{~W}$.

\subsubsection{Mechanisms}

The camera contains three electromechanical devices: an entrance aperture door, a filter wheel, and a detector channel (primary or secondary) selecting mirror.

The filter wheel is required to rotate five filters and a shutter into the optical path. The filter wheel location is shown in Figure 2.8.1.1. A stepper motor with fully redundant windings and position encoders is used for positioning. The 


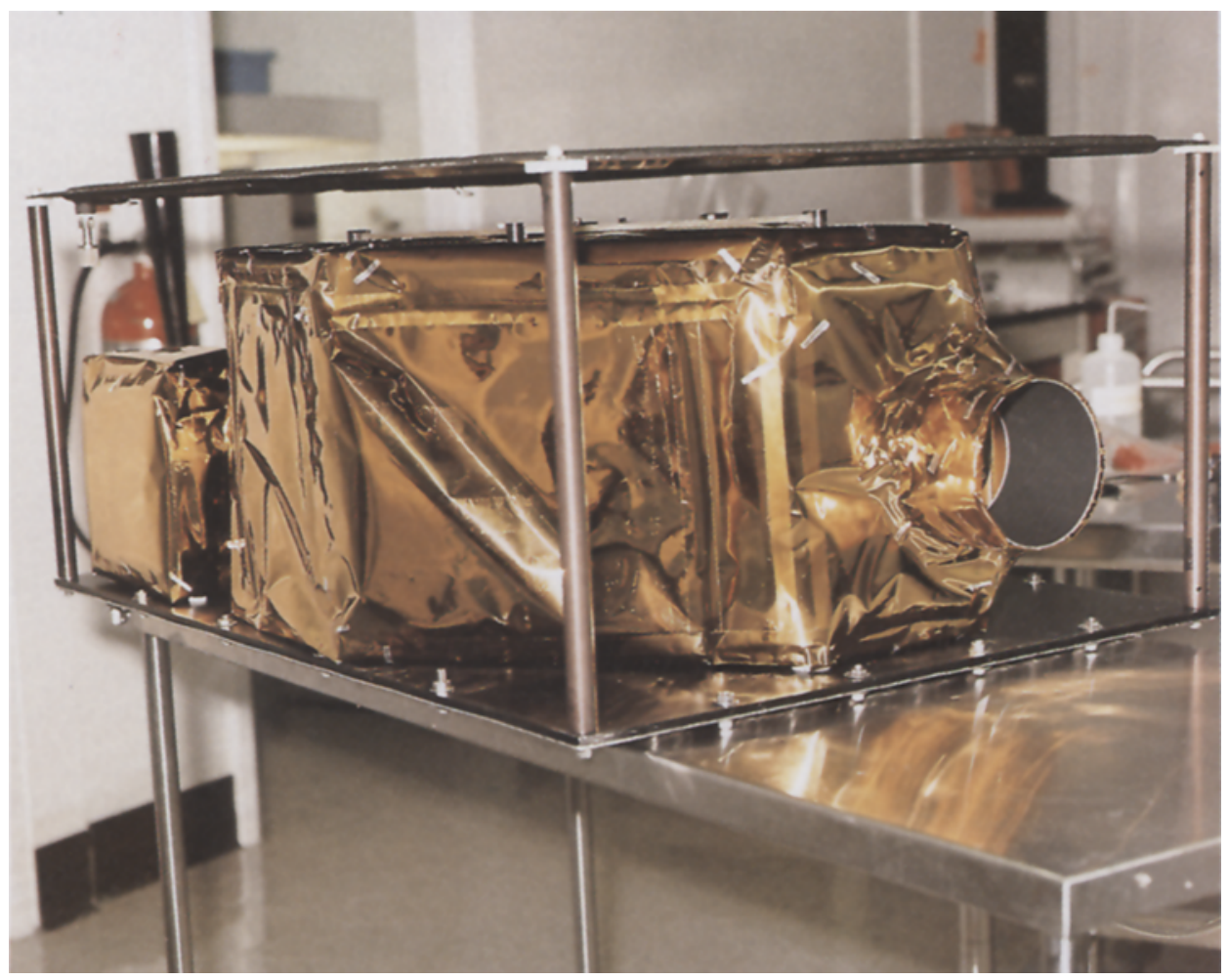

Fig. 2.8.2.1. Photograph of the Ultraviolet Imager mounted on fixture used for vacuum testing. The camera is the front box and most of the electronics is housed in the smaller box to the rear.

purpose of the entrance door is to close the instrument during non-operating conditions (integration, launch, thruster burns), and to provide some protection against contamination. It has a transparent $\mathrm{MgF}_{2}$ window in the event the redundant mechanism should fail in the closed position. A third device is a mirror mechanism which can be used to select the secondary detector. The aperture door and folding mirror are driven by hot paraffin motors which also incorporate redundant heater controls.

\subsubsection{Structure}

The instrument consists of two major components (at the black box level): the camera, and the electronics stack (Figure 2.8.2.1). The camera consists of a baseplate, one side of which interfaces with the despun platform. The optical bench and mechanisms are mounted to the other side of the baseplate. The camera is enclosed in a housing and above the housing, on the 'dark' side of the spacecraft, is the passive radiator. The radiator is covered with optical surface reflectors (OSRs). The optical bench is attached to the baseplate by a three point mount, and the three attach points have low thermal conductance. 
The electronics box comprises 9 crates into which the printed circuit boards of the command and data system are mounted. The connectors are all located on a connector plate at one end of this stack, and an end panel housing the low voltage power supplies completes the assembly. A specific area of the topside of the electronics stack is coated with Goddard Space Flight Center 'green' thermal paint to provide a thermal radiator.

Most of the structure is fabricated from magnesium in order to fit within the weight allocation.

\subsubsection{Thermal}

The principal requirements of the thermal design are as follows:

(a) The CCD chips must be cooled to $<-55^{\circ} \mathrm{C}$.

(b) The temperature of the optical bench must be maintained to $\pm 5^{\circ} \mathrm{C}$ of the design temperature in order to maintain alignment.

(c) Thermal gradients in the optical bench should not exceed $10{ }^{\circ} \mathrm{C}$ across the vertical dimension of the optical bench, and $5^{\circ} \mathrm{C}$ across the horizontal dimension in order to minimize optical distortions.

(d) The image intensifier should not be exposed to temperatures in excess of $55{ }^{\circ} \mathrm{C}$.

(e) The detector electronics should be maintained within a $10 \mathrm{C}$ window (although the window itself could lie between $-20^{\circ} \mathrm{C}$ and $+30^{\circ} \mathrm{C}$ ).

(f) The stack electronics should be maintained between $-20^{\circ} \mathrm{C}$ and $+40^{\circ} \mathrm{C}$.

A detailed multinode thermal model was developed for the instrument and showed that over the thermal extremes that the instrument will experience, the optical bench temperature will lie between $12{ }^{\circ} \mathrm{C}$ and $19{ }^{\circ} \mathrm{C}$, and the electronics stack will operate between $-10{ }^{\circ} \mathrm{C}$ and $40{ }^{\circ} \mathrm{C}$, which meets the goals. This is accomplished by a combination of thermal blankets, passive radiators, thermal surfaces, control heaters and isolation from the despun platform.

The CCD's are cooled by means of a passive radiator which is located on the side of the instrument that always faces away from the Sun. Similarly, the electronics stack radiates the heat generated within it from a surface on the dark side. Both the camera and the stack are thermally isolated from the despun platform and both are wrapped in thermal blankets. The optical bench is thermally isolated from the baseplate and housing. The baffle assembly is attached by means of low conductance material and the outside of the baffle is gold plated. Heaters are located on the baseplate and optical bench in order to provide thermal control.

Thermal tests have shown good agreement between the model and the measured temperatures over the anticipated mission extremes.

\subsection{COMMAND AND DATA SYSTEM}

A block diagram of the electronics is shown in Figure 2.9.1. 


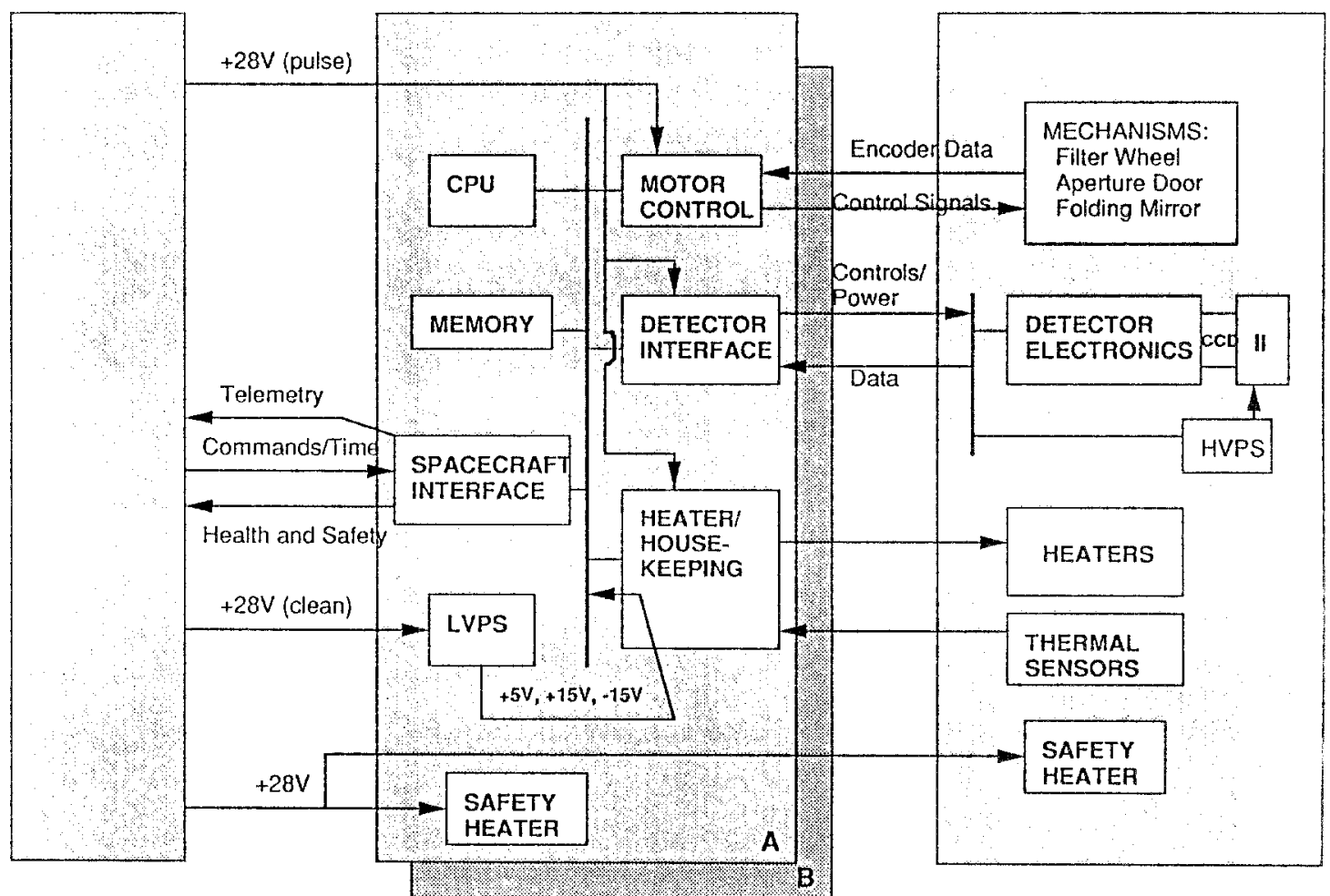

Fig. 2.9.1. Block diagram of the electronics.

The design of the camera control electronics is based on the Sandia 3300 microprocessor family. This 32 bit processor was selected because of its availability in a suitable radiation hardened form under a development effort by the ISTP project office. Interfaces from the SA3300 allow control of the mechanisms, intensifier gain, heaters, data readout and compression, as well as status read-back of bilevels, temperatures, MCP voltages, etc. Sufficient EPROM memory is included to store the basic default operational command tables for the instrument, and $4 \mathrm{Mbit}$ hybrid memory packs are used to store up to 4 full images. The electronics has two fully redundant systems each of which duplicates the functions shown in Figure 2.9.1. The instrument also has fully redundant interfaces to the spacecraft.

The data rate for the UVI instrument has been set by the ISTP project office to be $12 \mathrm{kbps}$. This data rate in turn establishes the frame rate for the instrument as follows: A single frame from the CCD comprises $244 \times 550$ pixels. When digitized to 12 bits this constitutes $1.6 \times 10^{6}$ bits. By adding 2 pixels in one dimension, as discussed above, by integrating on chip for $36.8 \mathrm{~s}$ ( 4 major frames), and by discarding portions of the array with no image (the rectangular ends and corners), 
TABLE 2.10 .1

Summary of instrument parameters

\begin{tabular}{ll}
\hline Wavelength range, $\AA$, (five filters) & $1300-1900$ \\
Focal length, mm & 124 \\
$f$ No. & 2.9 \\
Full aperture, $\mathrm{cm}^{2}$ & 11.75 \\
Solid angle (sr.) & $1.5 \times 10^{-2}$ \\
Field of view (full angle), degrees & 8 \\
Number of spatial elements & 36728 \\
Size of spatial elements at photocathode, $\mu \mathrm{m}$ & $74 \times 87$ \\
Typical sensitivity per spatial element (counts $/ R / 37 \mathrm{~s})$ & 0.1 \\
Angular resolution, deg & 0.036 \\
Data rate, kbps & 12 \\
Power, W & 21 \\
Mass, kg & 21 \\
\hline
\end{tabular}

the rate of $12 \mathrm{kbps}$ is reached. The detector system has been designed to be quite flexible, in that the on-chip integration period can be selected to be longer than the nominal $36.8 \mathrm{~s}$. In addition, smaller areas of the CCD can be read out at faster rates.

\subsection{SUMMARY OF INSTRUMENT}

A summary of the basic instrument parameters is given in Table 2.10.1.

\section{Calibration}

The instrument calibration must address several aspects of the instrument performance and behavior. We need to be able to relate the measured output in digital words (DW) to an absolute brightness scale. We need to be able to correct this for the particular gain level (MCP voltage) at which the measurement was made. The linearity of the response (or departures therefrom) as a function of signal intensity must be known. We must also quantify any angular dependence of the response across the field of view, and have information that will allow us to relate measurements made at different detector temperatures, as the cold temperature reached by the radiator and CCD's will vary over the course of the mission. In addition to the above, the detector actually consists of almost 37000 discrete detector elements, each of which has its own behavior. It is therefore important to be able to quantify the response when the instrument field of view is filled by a suitable 'flat-field' source. As a result, the calibration of the instrument represents a substantial effort that must be carefully and rigorously carried out as the subsequent data products 
can be no better than the calibration. To adequately calibrate such an instrument (to accuracies of $\pm 10 \%$ ) requires particular care and some new technology. The details are discussed elsewhere (Torr and Zukic, 1995) and only an outline is given here.

At the time of writing, a preliminary calibration has been made of the flight instrument. The final calibration will be made a few months before launch and will address some aspects not yet covered. The calibration work is done in a vacuum ultraviolet calibration facility (Torr and Vitz, 1982) that allows illumination of the instrument field of view with various UV light sources. The VUV calibration facility is fitted with $\mathrm{LN}_{2}$ baffles which allow the passive radiators on the instrument to reach their operating temperatures and thus cool the CCD and electronics to their required ranges. The lamps used to calibrate the instrument include deuterium and various hollow cathode lamps.

In order to characterize the spectral response as a function of wavelength, a collimated beam of monochromatic light is directed into the instrument. This beam (approximately $1 \mathrm{~cm}^{2}$ in cross section) can be scanned in angle so as to illuminate different parts of the field of view. The beam can be scanned in wavelength over the entire range of the instrument. Using this system, measurements were made with each filter in the optical path, and at several different angles in the field of view. The instrument response at each wavelength is compared with NBS calibrated diodes/PMTs mounted inside the system by using a gimbal control to move the beam from the instrument to the PMT, thus placing the response on an absolute scale. If time permits, the spectral response will also be characterized using an electron-impact-on-flowing-gas source (Ajello et al, 1987) that produces the LBH, $\mathrm{OI}, \mathrm{NI}$ and $\mathrm{HI}$ features in the same manner as they are generated in the aurora.

To characterize the pixel to pixel response of the instrument requires a VUV flat field source, an item that is not commercially available and difficult to produce. After several failed attempts to make a satisfactory flat field source, we were finally able to come up with a design that met our requirement of $1 \%$ uniformity (Torr and Zukic, 1995) that will be used in the pre-launch calibration. Using this source, not only is the pixel to pixel variability characterized, but the entire field of view is illuminated (not possible with the pencil-like collimated beam used to map the wavelength response), and the dependence on gain step is determined.

After launch, a partial calibration of the instrument will be attempted by routinely looking at known intensity FUV stars. While this approach has its limitations, it will give some information on the long-term stability of the instrument but would be quite inadequate as the only calibration.

\section{Operations}

In considering the operational sequences to be carried out by the UVI, two factors are particularly important: The spacecraft will pass over ground tracking stations at 


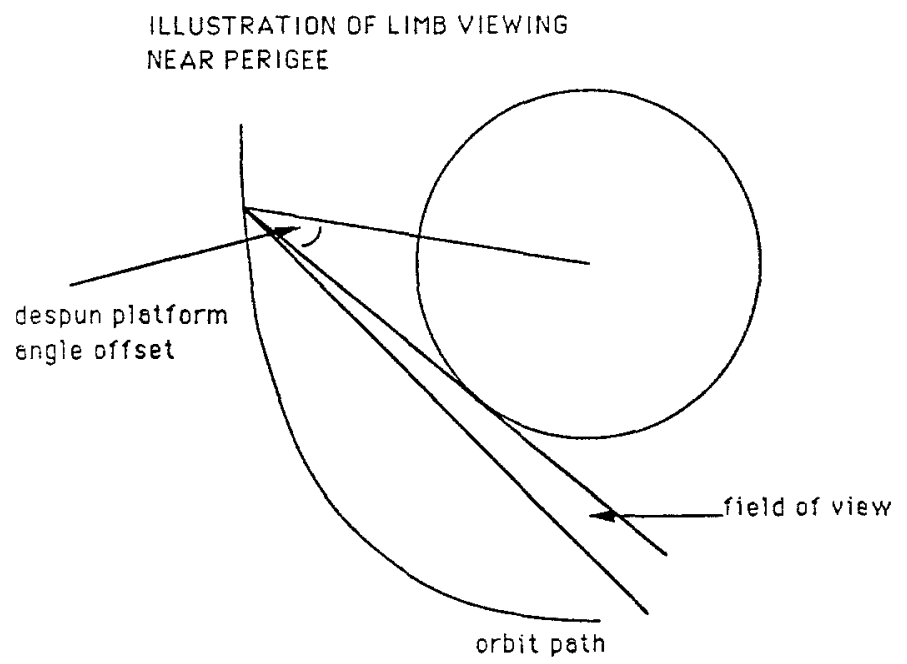

Fig. 4.1. Illustration of limb viewing mode to obtain images of the aurora in altitude.

most 4 times a day. Thus no operations (except those of an emergency nature) are real time. Secondly, operations will be planned for a one week period, approximately two weeks in advance of their implementation. This means that the observing sequences must be designed to deal with a fairly broad range of geophysical possibilities without intervention.

The UVI observing sequences are built in the form of command tables, each for a specific observing mode. These tables are keyed to location in the orbit. For example, one of the prime observing sequences is that to be run for spacecraft distances greater than $6 R_{\mathrm{E}}$ geocentric. In this mode the instrument will cycle through various selected filter combinations required to permit observations of the spectral features needed for a particular objective. The integration times spent on each filter are adjusted appropriately according to the anticipated typical brightness of the features. Each filter cycle is typically preceded by an instrument background measurement. In its most complete form, this sequence has a repeat time of $10 \mathrm{~min}$. For many purposes it will not be necessary to make measurements with every filter on every spectral cycle. A cycle time of $4 \mathrm{~min}$ is representative of a typical mode. Where temporal details are important, the instrument can be operated in a single filter mode, permitting the 37-s resolution.

Closer to the Earth, images will be obtained with smaller spatial coverage but with higher spatial resolution. Near perigee an observing mode is planned with the despun platform offset to allow observing on the limb of the Earth to obtain 'altitude' images of the aurora. This is illustrated in Figure 4.1. In this mode, for example, from a distance of $1.8 R_{\mathrm{E}}$ the field of view will span $\sim 1000 \mathrm{~km}$ radially out from the Earth, at approximately $5 \mathrm{~km}$ per pixel. 
Observations of stars will be scheduled from time to time for the purpose of obtaining calibration information using UV stars of known intensity, and to provide information on the pointing of the despun platform to a finer resolution than is available with the spacecraft provided systems.

\section{Data Products}

The UVI will produce an average of 3000 images a day. After these are processed, with backgrounds subtracted and a certain amount of coherent summing at times of very weak intensities, the product will be approximately 2000 images a day. The raw data will be received by the Central Data Handling Facility (CDHF) at the Goddard Space Flight Center. There they will undergo processing to the point that the key parameters are extracted. The latter are a set of geophysical parameters that will be stored in a common file accessible to all investigators, and can be used for searching through the database for periods of interest to particular studies. Because of the volume of data associated with an imager (the UVI will produce approximately one million images in the course of the nominal mission), much of the remaining processing of the images and the scientific studies with these will be done on computer facilities at our institutions, which form a Remote Data Acquisition Facility (RDAF). The processing at either the CDHF or the RDAF will be done using algorithms developed by the UVI team.

The division of processing tasks is shown in Figure 5.1. The CDHF will strip out the key parameters from the images. These will take the form of the total energy flux and the characteristic energy, each of which will be determined for four quadrants: the noon, midnight, dawn and dusk sectors of the auroral oval. An estimate will also be made for the polar cap. A parameter will be generated to indicate the level of activity and the most equatorward and most poleward boundary of the aurora will be provided. In addition to these single number parameters, one image every 10 min will be stored in the CDHF for browsing purposes.

The processing of the parameter images will be done on the RDAF. These will be 2-D images of the energy flux, the characteristic energy, and the ionospheric conductance, all determined from the various wavelength ratios, as well as monochromatic temporal/spatial images. The RDAF will also store the special mode images, such as the limb viewing sequences, dayglow images and high resolution images. The RDAF will contain a database of the image products that can be queried according to a broad list of sorting parameters.

\section{Summary}

In the design of the Ultraviolet Imager, we have placed high priority on two things: simultaneously acquired global images, and images that can be quantitatively 


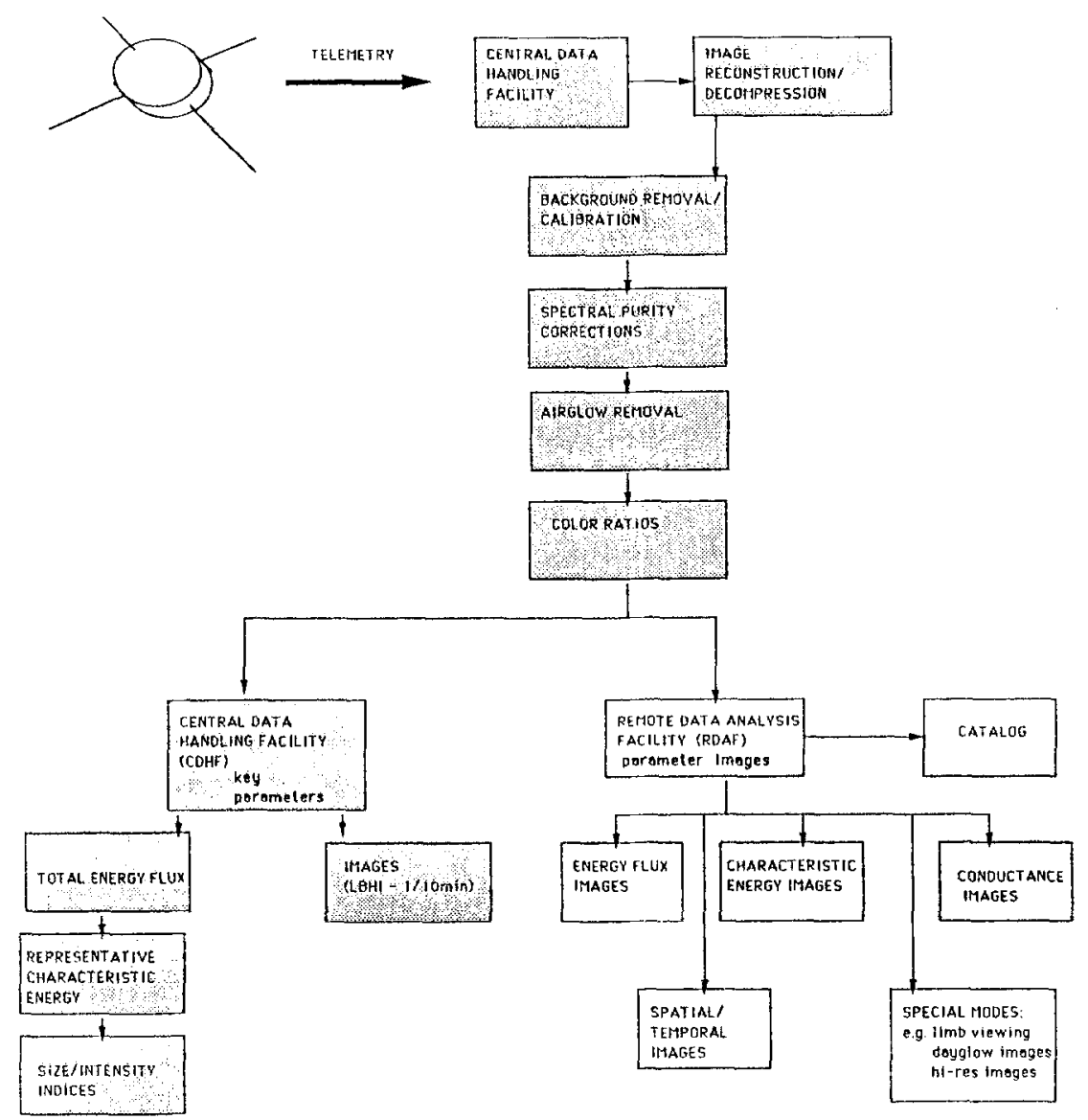

Fig. 5.1. Illustration of data products and processing distribution.

interpreted to yield the parameters of interest. The first of these principal goals requires a field of view sufficiently large to image the entire oval over a significant part of the highly elliptic orbit, a despun platform to allow continuous viewing of the oval, and a suitable 2-D focal plane array detector on which the oval can be imaged. The second of these goals has required that we advance the state-of-the-art of FUV filter technology in order to be able to accurately separate the weak features of diagnostic interest from the very much brighter neighboring contaminants, while at the same time blocking the huge component of visible scattered sunlight. In addition, particular care was needed in planning for the unusual calibration needs of the instrument. The fact that the features of most use diagnostically are relatively weak, also required that the optical system be fast and yet capable of good imaging over the large field of view. The dynamic range of the aurora from one integration time to the next, and also within a single image, placed severe demands on the optics (the surfaces must be sufficiently smooth to prevent scattering of the energy in the image to other parts of the image) and on the detector system (which must 
be capable of spanning a large dynamic range and have highly stable gain control). The capture and storage of the images to fit within spacecraft telemetry resources has driven the design of the command and data system. The instrument described here has met all of these requirements (with the exception of a major part of the calibration which has yet to be done) and we hope it will allow us to enter the ISTP mission phase with a new and powerful imaging capability.

The substantial technological advances that are exploited in this instrument greatly enhance what can now be achieved in terms of far ultraviolet imaging. The simultaneous acquisition of the auroral images meets the global coherence emphasis of the ISTP program. The ability to quantitatively separate spectral features means that where previously, 60 to $80 \%$, or more, of the measured signal was contaminant, now more than $90 \%$ of the signal is the feature of interest. New approaches to optical design have resulted in significant improvements in the instrument speed and hence sensitivity, thus lowering the threshold of the intensities of auroral features that can be seen. As a result, we will now be able to explore details in what appeared previously as 'dark' regions in auroral images. Filters with sufficient blocking of long wavelength sunlight will allow the first imaging of these spectrally pure FUV fully sunlit midday auroras. Current approaches to fabrication of optical components permits surfaces to be produced that are sufficiently smooth to prevent degradation in performance through scattering of energy. New design techniques have allowed us to produce a FUV flat field calibration source that meets the uniformity and brightness requirements.

In parallel with the instrument design and development, we have invested a comparable effort in modeling and interpretation of the auroral ionosphere and thermosphere. This has provided the basis for the key parameter and parameter image extraction.

All of these factors taken together, mean that we should be able to take a fundamental step forward in our ability to acquire and transform auroral images into images of primary parameters that describe both the precipitating particles and their impact on the ionosphere.

\section{Acknowledgements}

A large number of people have contributed significantly to the development of the UVI since its inception in 1979. Dr Karl Guenther first suggested that we develop the narrow band FUV filters. The final optical design optimization was done by Dr Feng. Drs M. Krim and A. Nonemacher provided the optical bench assembly concept and the initial baffle assessments. The surface roughness requirements as illustrated in Figure 2.3.3 were quantified by Mr J. Harvey. Mr C. Fellows played a key role in the assembly of the camera system. Dr J. Spann played a key role in the coordination of the development. Messrs Savage, Clem, Tejada, Fellows, Dougani, Baldridge, Shimizu, and McGowan have all made substantial contributions to 
the command and data system. Dr A. Ahmad and Mr G. Loughead have been responsible for the mechanical design. Dr. C. Keffer played an important role in setting up the VUV test facilities and conducting the filter tests. Dr J. Kim assisted in the fabrication of the filters. Excellent support has been provided throughout the design and development of the instrument by the ISTP Mission Manager, Mr John Hrastar and his predecessor, Mr K. Sizemore, and the GGS team. Much of the work reported here was done under NASA Contracts NAS8-38145 to the University of Alabama in Huntsville and NAS8-38146 to Science and Engineering Associates, Inc.

\section{References}

Ajello, J. M. and Shemansky, D. E.: 1985, 'A Reexamination of Important $\mathrm{N}_{2}$ Cross Sections by Electron Impact with Application to the Dayglow: The Lyman-Birge-Hopfield Band System and NI $(119.99 \mathrm{~nm})$ ', J. Geophys. Res. $90,9845$.

Ajello, J. M., Shemansky, D. E., Franklin, B., Watkins, J., Srivastava, S., James, G. K., Simms, W. T., Hord, C. W., Pryor, W., McClintock, W., Argabright, V., and Hall, D.: 1987, 'A Simple Ultraviolet Calibration Source with Reference Spectra and Application to the Galileo Orbiter Ultraviolet Spectrometer', Appl. Opt. 27, 890.

Akasofu, S.-I.: 1981, in S.-I. Akasofu and J. R. Kan (eds.), Physics of Auroral Arc Formation, Auroral Arcs and Auroral Potential Structure, AGU Geophysical Monograph, Washington D.C.

Allen, C. W., 1973, Astrophysical Quantities, Athlone Press, University of London.

Anger, C. D., Fancott, T., McNally, J., and Kerr, H. S.: 1973, 'ISIS-II Scanning Auroral Photometer', Appl. Opt. 12, 1753.

Anger, C. D., Babey, S. K., Broadfoot, A. L., Brown, R. G., Cogger, L. L., Gattinger, R., Haslett, J. W., King, R. A., McEwen, D. J., Murphree, J. S., Richardson, E. H., Sandel, B. R., Smith, K., and Vallance Jones, A.: 1987, 'An Ultraviolet Auroral Imager for the Viking Spacecraft', Geophys. Res. Letters 14, 387.

Barth, C. A.: 1966, in A. Green (ed.-), The Middle Ultraviolet: Its Science and Technology, The Ultraviolet Spectroscopy of Planets, John Wiley and Sons, New York.

Cook, I. G.: 1987, in C. Londono and R. I. Fischer (eds.), 'Recent Trends in Optical Systems Design: Computer Lens Design Workshop, Wide Field of View Three-Mirror Anastigmatic (TMA) Employing Spherical Secondary and Tertiary Mirrors', Proc. SPIE, 766, 158.

Fennelly, J. A., Tor, D. G., Richards, P. G., and Torr, M. R.: 1994, 'Simultaneous Retrieval of the Solar EUV Flux and Neutral Thermospheric $\mathrm{O}, \mathrm{O}_{2}, \mathrm{~N}_{2}$ and Temperature from Twilight Airglow', J. Geophys. Res. 99, 6483.

Frank, L. A., Craven, J. D., Ackerson, K. L., English, M. R., Eather, R. H., and Carovillano, R. L.: 1981, 'Global Auroral Imaging Instrumentation for the Dynamics Explorer Mission', Space Sci. Instr. 5, 369.

Germany, G. A., Torr, M. R., Richards, P. G., and Torr, D. G.: 1990, 'Dependence of Modeled O I 1356 and $\mathrm{N}_{2}$ LBH Auroral Emissions on the Neutral Atmosphere', J. Geophys. Res. 95, 7725.

Germany, G. A., Torr, M. R., Torr, D. G., and Richards, P. G.: 1994a, 'The Use of FUV Auroral Emissions as Diagnostic Indicators', J. Geophys. Res. 99, 383.

Germany, G. A., Torr, D. G., Torr, M. R., and Richards, P. G.: 1994b, 'The Determination of Ionospheric Conductances from FUV Auroral Images', J. Geophys. Res. 99, 383.

Heroux, L. and Swirbalus, R. A.: 1976, J. Geophys. Res. 81, 436.

Hirao, K. and Itoh, T.: 1978, 'Scientific Satellite Kyokko (EXOS-A)', Solar Terrest. Env. Res. in Japan 2, 148.

Johnson, R. B.: 1988, 'Wide Field of View Three-Mirror Telescopes Having a Common Optical Axis', Opt. Eng. 27, 1046.

Kamide, Y. and Richmond, A. D.: 1982, 'Ionospheric Conductivity Dependence of Electric Fields and Currents Estimated from Ground Magnetic Observations', J. Geophys. Res. 87, 8331. 
Kaneda, E., Takgi, M., and Niwa, N.; 1977, 'Vacuum Ultraviolet Television Camera', Proc. 12th Int. Symp. Space Tech. Sci., Agne, Tokyo, pp. 233-238.

Keffer, C. E., Torr, M. R., Zukic, M., Spann, J. F., Torr, D. G., and Kim, J.: 1993, 'Radiation Damage Effects in Far Ultraviolet Filters and Substrates', SPIE Vol. 2018, Passive Materials for Optical Elements II, p. 92.

Korsch, D.: 1975, 'Three Mirror Space Telescope', Opt. Eng. 14, 533.

Korsch, D.: 1977, 'Anastigmatic Three-Mirror Telescope', Appl. Opt. 16, 1074.

Korsch, D.: 1980, 'Design and Optimization Technique for Three-Mirror Telescopes', Appl. Opt. 19, 3640 .

Meng, C. I. and Huffman, R. E.: 1984, 'Ultraviolet Imaging from Space of the Aurora Under Full Sunlight', Geophys. Res. Letters 11, 315.

Oguti, T., Kaneda, E., Ejiri, M., Sasaki, S., Kadokura, A., Yamamoto, T., Hayashi, K., Fujii, R., and Makita, K.: 1990, 'Studies of Aurora Dynamics by Aurora-TV on the Akebono (EXOS-D) Satellite', J. Geomag. Geoelectr: 42, 555.

Richards, P. G.: 1991, 'An Improved Algorithm for Determining Neutral Winds from the Height of the F2 Peak Electron Density', J. Geophys. Res. 96, 17839.

Richards, P. G., Fennelly, J., and Torr, D. G.: 1994, 'EUVAC: Solar EUV Flux Model for Aeronomic Calculations', J. Geophys. Res. 99, 8981.

Rogers, E. H., Nelson, D. F., and Savage, R. C.: 1974, 'Auroral Photography from a Satellite', Science $183,951$.

Torr, M. R.: 1985, Persistence of phosphor glow in microchannel plate image intensifiers, Appl. Opt., 24,793 .

Torr, M. R. and Devlin, J.: 1982, 'Intensified Charge Coupled Device for Use as a Spaceborne Spectrographic Image Plane Detector System', Appl. Opt. 21, 3091.

Torr, M. R. and Torr, D. G.: 1995, 'A Compact Imaging Spectrometer for Broadband Spectral Simultaneity', Appl. Opt. (in press).

Torr, M. R. and Vitz, R. C.: 1982, 'An Extreme Ultraviolet Imaging Spectrometer for Thermospheric Emissions', Appl. Opt. 21, 3080.

Torr, D. G. and Zukic, M.: 1995, 'Absolute Calibration of a Focal Plane Detector System in the FUV', Appl. Opt. (submitted).

Torr, D. G., Torr, M. R., Zukic, M., Spann, J., and Johnson, R. B.: 1993, 'Ultraviolet Imager for the International Solar Terrestrial Physics Mission, Optical Engineering 32, 3060.

Torr, M. R., Torr, D. G., and Hinteregger, H. E.: 1980, 'Solar Flux Variability in the Schumann-Runge Continuum as a Function of Solar Cycle 21', J. Geophys. Res. 85, 6063.

Torr, M. R., Torr, D. G., Baum, R., and Spielmaker, R.: 1986, 'Intensified-CCD Focal Plane Detector for Space Applications: A Second Generation', Appl. Opt. 25, 2768.

Torr, M. R., Torr, D.G., Chang, T., Richards, P., Swift, W., and Li, N., 1994, Thermospheric and mesospheric nitric oxide from the ATLAS-1 and Spacelab 1 missions, J. Geophys. Res. (submitted), June.

Torr, M.R ., Torr, D. G., Chang, T., Richards, P., and Germany, G.: 1994c, 'The $\mathrm{N}_{2}$ Lyman-BirgeHopfield Dayglow from ATLAS-1', J. Geophys. Res. 99, 21397.

Zukic, M., Torr, D. G., Spann, J. F., and Torr, M. R.: 1990a, 'VUV Thin Films, Part I: Optical Constants of $\mathrm{BaF}_{2}, \mathrm{CaF}_{2}, \mathrm{LaF}_{2}, \mathrm{MgF}_{2}, \mathrm{Al}_{2} \mathrm{O}_{3}, \mathrm{HfO}_{2}$ and $\mathrm{SiO}_{2}$, Appl. Opt. $29,4284$.

Zukic, M., Torr, D. G., Spann, J. F., and Torr, M. R.: 1990b, 'VUV Thin Films, Part II: Vacuum Ultraviolet-Dielectric Narrowband Filters', Appl. Opt. 29, 4293.

Zukic, M., Torr, D. G., Kim, J., Spann, J., and Torr, M. R.: 1993, 'Filters for the International Solar Terrestrial Physics Mission Far-Ultraviolet Imager', Optical Engineering 32, 3069. 